\title{
Site-Selective Functionalization of Methionine Residues via Photoredox Catalysis
}

\author{
Junyong $\mathrm{Kim}^{\dagger}$, Beryl X. $\mathrm{Li}^{\dagger}$, Richard Y.-C. Huang ${ }^{\ddagger}$, Jennifer X. Qiao ${ }^{\Uparrow}$, William R. Ewing
} David W. C. MacMillan*广

\footnotetext{
${ }^{\dagger}$ Merck Center for Catalysis at Princeton University, Princeton, New Jersey 08544, United States

$\$$ Pharmaceutical Candidate Optimization, Research and Development, Bristol-Myers Squibb Company, Princeton, New Jersey 08542, United States

"Discovery Chemistry, Research and Development, Bristol-Myers Squibb Company, Princeton, New Jersey 08542, United States
}

*e-mail: dmacmill@princeton.edu 
$\begin{array}{ll}\text { 1. General information } & S 3\end{array}$

2. Optimization study and control experiments $\quad S 4$

$\begin{array}{lc}\text { 2.1. Solid phase peptide synthesis } & S 4\end{array}$

2.2. General reaction conditions and characterization method $S 4$

$\begin{array}{ll}\text { 2.3. Optimization tables and control experiments } & S 5\end{array}$

3. Modification of aprotinin with various Michael acceptors $\quad S 8$

$\begin{array}{lc}\text { 3.1. Reaction conditions and characterization methods } & S 8\end{array}$

3.1.1. General reaction conditions for methionine alkylation on aprotinin $\quad S 8$

$\begin{array}{lc}\text { 3.1.2. Proteomics analysis } & S 8\end{array}$

$\begin{array}{lr}\text { 3.2. Michael acceptor substrates } & S 10\end{array}$

$\begin{array}{ll}\text { 4. Protein scope and characterization } & S 23\end{array}$

$\begin{array}{lr}\text { 4.1. Reaction conditions and characterization methods } & S 23\end{array}$

4.1.1. General reaction conditions for methionine alkylation on protein substrates $\quad S 23$

$\begin{array}{ll}\text { 4.1.2. Proteomics analysis } & S 23\end{array}$

$\begin{array}{lr}\text { 4.2. Protein substrates } & S 25\end{array}$

$\begin{array}{ll}\text { 4.2.1. Ubiquitin } & S 25\end{array}$

$\begin{array}{lr}\text { 4.2.2. } \alpha \text {-lactalbumin } & \text { S27 }\end{array}$

$\begin{array}{lr}\text { 4.2.3. Myoglobin } & S 29\end{array}$

4.2.4. Human growth hormone (hGH) $\quad S 32$

$\begin{array}{ll}\text { 4.2.5. Carbonic anhydrase (CA) } & S 36\end{array}$

$\begin{array}{ll}\text { 4.2.6. Ribonuclease A } & S 40\end{array}$

$\begin{array}{lr}\text { 4.2.7. EGFP } & S 43\end{array}$

$\begin{array}{lr}\text { 5. Modification of EGFP } & S 48\end{array}$

$\begin{array}{lr}\text { 5.1. Experimental procedures } & S 48\end{array}$

5.1.1. General reaction conditions for methionine alkylation on EGFR S48

$\begin{array}{lr}\text { 5.1.2. Click reactions on alkyne attached EGFP } & S 48\end{array}$

$\begin{array}{ll}\text { 5.1.3. Cell culture and imaging } & S 48\end{array}$

5.2. Intact mass analysis of EGFP-alkyne (26) $\quad S 49$

5.3. Western blot analysis of modified EGFP $\quad S 50$

5.4. Confocal microscopy images of protein internalization to SJSA-1 cells S50

6. Experimental procedures for the preparation of Michael acceptors S51

7. NMR spectra $\quad S 55$

8. Cyclic voltammetry data of amino acids $\quad S 61$

$\begin{array}{lr}\text { 9. References } & \text { S64 }\end{array}$ 


\section{General information}

Reagents. All commercial reagents were used as received without further purification. Lumiflavin was purchased from Santa Cruz Biotechnology (Cat\# sc-224045). Aprotinin was purchased from G-Biosciences (Cat\# 786-046). Recombinant human growth hormone was purchased from Genscript (Cat\# Z03012). Recombinant enhanced green fluorescent protein (EGFP) was purchased from Chromotek (Cat\# EGFP-250). All remaining proteins investigated (ubiquitin (Cat\# U6253), $\alpha$-lactalbumin (Cat\# L5385), myoglobin (Cat\# M0630), carbonic anhydrase (Cat\# C3934), ribonuclease A (Cat\# R6513)) were purchased from Sigma Aldrich in lyophilized form. Protein structures were obtained from the Protein Data Bank (rcsb.org). Milli-Q ultrapure water purified through Millipore Milli-Q Advantage A1 purification system was used for all bioconjugation reactions. Small molecule reagents and buffer salts were purchased from Sigma Aldrich and used without purification.

Photoredox light set-up. Photoredox reactions were performed with Kessil PR160 LED Photoredox Lights, $440 \mathrm{~nm}$ blue light.

Protein purification. Protein reactions were purified via one of the following: protein concentrators (Pierce, 3K MWCO, $0.5 \mathrm{~mL}$ ), spin desalting columns (Zeba, 7K MWCO, $0.5 \mathrm{~mL}$ ), or semi-preparatory HPLC method. Protein samples were lyophilized with a Millrock Technology MD 85 lyophilizer.

HPLC. HPLC analyses and purifications were performed on an Agilent 1260 Infinity LC system using an Agilent Eclipse Plus C18 column (3.5 $\mu \mathrm{m}, 4.6 \mathrm{~mm}$ x 100 mm, part number 959961-902).

Mass spectrometry. High Resolution Mass Spectra were obtained from the Princeton University Mass Spectral Facility. For intact protein LC-MS, samples were desalted and eluted from a reversed-phase C18 Scaling column (ACQUITY UPLC BEH C18 column 300A 1.7um 2.1x150mm). For LC-MS/MS peptide mapping analysis, digested peptides were separated on a reversed-phase C18 column (ACQUITY UPLC BEH C18 column 130A 1.7um 2.1x150mm). Data was acquired on a QEPlus mass spectrometer (ThermoFisher Scientific, Waltham, MA) connected to Aquity UPLC (Waters, Milford, MA), and analyzed using Thermo BioPharma Finder software (ThermoFisher Scientific, Waltham, MA).

NMR spectroscopy. ${ }^{1} \mathrm{H}$ NMR and ${ }^{13} \mathrm{C}$ NMR spectra were recorded on Bruker UltraShield Plus Avance III 500 $\mathrm{MHz}$ and are internally referenced to residual solvent signals: $\mathrm{CDCl}_{3}(7.26 \mathrm{ppm}$ and $77.0 \mathrm{ppm}$, respectively). Data

for ${ }^{1} \mathrm{H}$ NMR are reported as follows: chemical shift $(\delta \mathrm{ppm})$, multiplicity $(\mathrm{s}=$ singlet, $\mathrm{d}=$ doublet, $\mathrm{t}=$ triplet, $\mathrm{q}=$ quartet, quint $=$ quintet, $\mathrm{m}=$ multiplet, $\mathrm{dd}=$ doublet of doublets, $\mathrm{td}=$ triplet of doublets, $\mathrm{br}=$ broad), coupling constant $(\mathrm{Hz})$, and integration.

Cyclic Voltammetry. All CV experiments were performed on CH Instruments Electrochemical Workstation (Austin, TX). Data acquisition was performed on CHI software (Ver14.05). 


\section{Optimization study and control experiments}

\subsection{Solid phase peptide synthesis}

Solid phase peptide synthesis was performed on 2-chlorotrityl chloride resin (100-200 mesh, 1-2 meq/g, crosslinked polystyrene) manually. 2-chlorotrityl chloride resin, 2-(1H-benzotriazol-1-yl)-1,1,3,3tetramethyluronium hexafluorophosphate (HBTU), 1-hydroxybenzotriazole hydrate (HOBt), and N,Ndiisopropylethylamine (DIPEA) were purchased from Chem Impex Int'1 Inc. 1,1,1,3,3,3-hexafluoro-2-propanol (HFIP) was purchased from Oakwood Chemical. Synthesized peptides were purified by reverse phase Biotage using an Isolera One instrument with Biotage snap cartridge KP-C-18-HS columns.

\subsection{General reaction conditions and characterization method}

Milli-Q water was degassed by the freeze-pump-thaw method (5 cycles). All stock solutions in the method were made with degassed solvents and stored in a glovebox. To an 8 dram vial containing magnetic stir bar was added tetrapeptide solution $\left(50 \mathrm{mM}\right.$ in $\mathrm{H}_{2} \mathrm{O}, 100 \mu \mathrm{L}, 5 \mu \mathrm{mol}, 1$ equiv. $)$, photocatalyst solution $\left(5 \mathrm{mM}^{\mathrm{in}} \mathrm{H}_{2} \mathrm{O}, 100\right.$ $\mu \mathrm{L}, 0.5 \mu \mathrm{mol}, 10 \mathrm{~mol} \%$ ), diethyl ethylidenemalonate (2, 9.1 $\mu \mathrm{L}, 50 \mu \mathrm{mol}, 10$ equiv.), PBS stock solution (Gibco, 10x, pH 7.4, $100 \mu \mathrm{L})$, and Milli-Q water $(700 \mu \mathrm{L})$. The vial was sealed and brought out of the glovebox. The reaction was stirred and irradiated using a $40 \mathrm{~W}$ Kessil blue LED lamp (PR160-440, 100\% intensity, $4 \mathrm{~cm}$ away) for $30 \mathrm{~min}$. After reaction completion, the solution was added a $p$-toluic acid solution $(100 \mu \mathrm{L}, 10 \mathrm{mM}$ in methanol) as an internal standard. An aliquot of the mixture was taken for HPLC analysis.

HPLC analyses were performed on an Agilent 1260 Infinity LC system using a reverse-phase C18 column (Agilent Zorbax 300SB-C18, $3.5 \mu \mathrm{m}, 100 \mathrm{~mm}$ ). The eluents used were Buffer A (water $+0.1 \%$ TFA) and Buffer B (9:1 acetonitrile: water + 0.1\% TFA). HPLC method: 1 min 100\% Buffer A, followed by 0-73\% gradient of Buffer B over $25 \mathrm{~min}$, followed by 2 min flush at $90 \%$ buffer B, followed by 2 min re-equilibration to $100 \%$ buffer A (water $+0.1 \%$ TFA). Yield was determined by taking the ratio of $\left[\mathrm{A}_{\text {substrate }} / \mathrm{A}_{\text {standard }}\right] /\left[\mathrm{A}_{\text {product }} / \mathrm{A}_{\text {standard }}\right]$ where $\mathrm{A}_{\text {substrate }}$ $=$ area in $\mathrm{mAU}$ of the substrate peak, $\mathrm{A}_{\text {product }}=$ area in $\mathrm{mAU}$ of the product peak, and $\mathrm{A}_{\text {standard }}=$ area in $\mathrm{mAU}$ of the internal standard. The ratio of $\left[\mathrm{A}_{\text {substrate }} / \mathrm{A}_{\text {standard }}\right]$ was measured from a solution containing $5 \mathrm{mM}$ of the tetrapeptide substrate and $1 \mathrm{mM}$ of the internal standard.

Note: Solutions can also be degassed by sparging with nitrogen for 10 min. Reaction mixture prepared with such degassed solvents provided peptide products without significant oxidation of peptides. Also, DMSO can be utilized as an organic solvent instead of DMF, albeit resulting in slightly lower conversions. 


\subsection{Optimization tables and control experiments}

Table S1. Evaluation of photocatalysts in the photoredox methionine-selective modification.

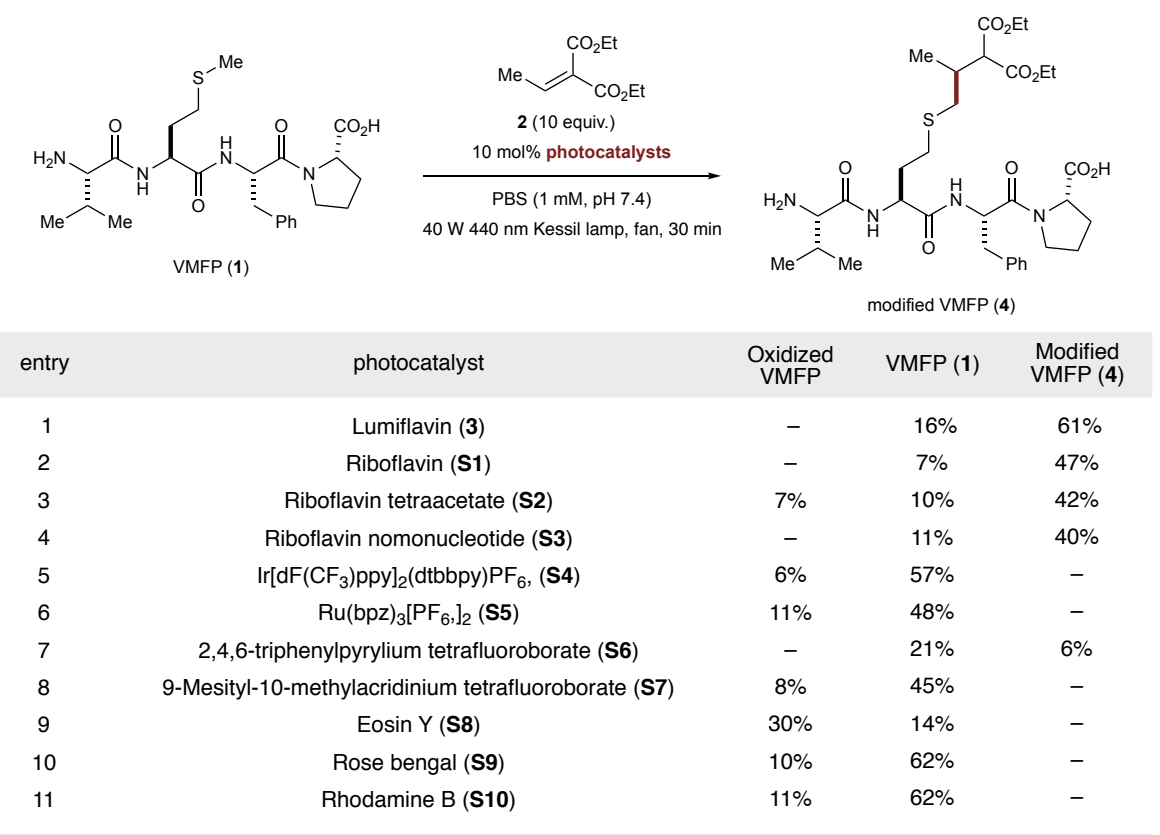
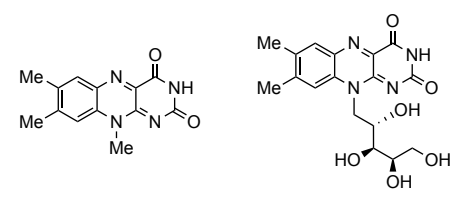

Lumiflavin (3)

Riboflavin (S1)

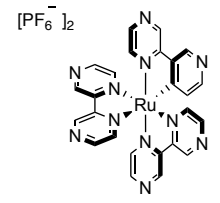

$\mathrm{Ru}(\mathrm{bpz})_{3}\left[\mathrm{PF}_{6},\right]_{2}(\mathbf{S} 5)$

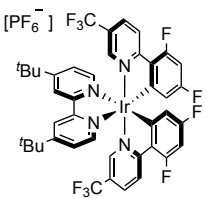

$\operatorname{Ir}\left[\mathrm{dF}\left(\mathrm{CF}_{3}\right) \mathrm{ppy}_{2}\right]_{2}(\mathrm{dtbbpy}) \mathrm{PF}_{6},(\mathbf{S} 4)$

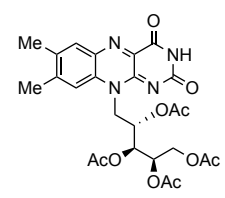

Riboflavin tetraacetate (S2)

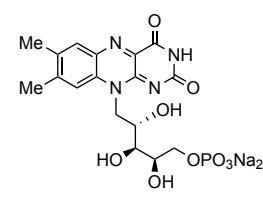

Riboflavin mononucleotide (S3)

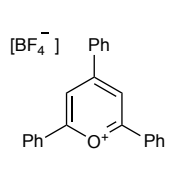

2,4,6-triphenylpyrylium

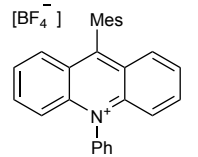

9-Mesityl-10-methylacridinium tetrafluoroborate (S7)

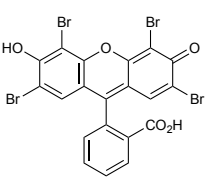

Eosin Y (S8)

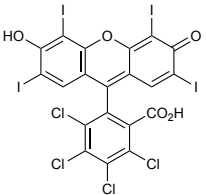

Rose bengal (S9)

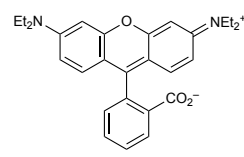

Rhodamine B (S10) 
Figure S1. HPLC trace from the optimized reaction.
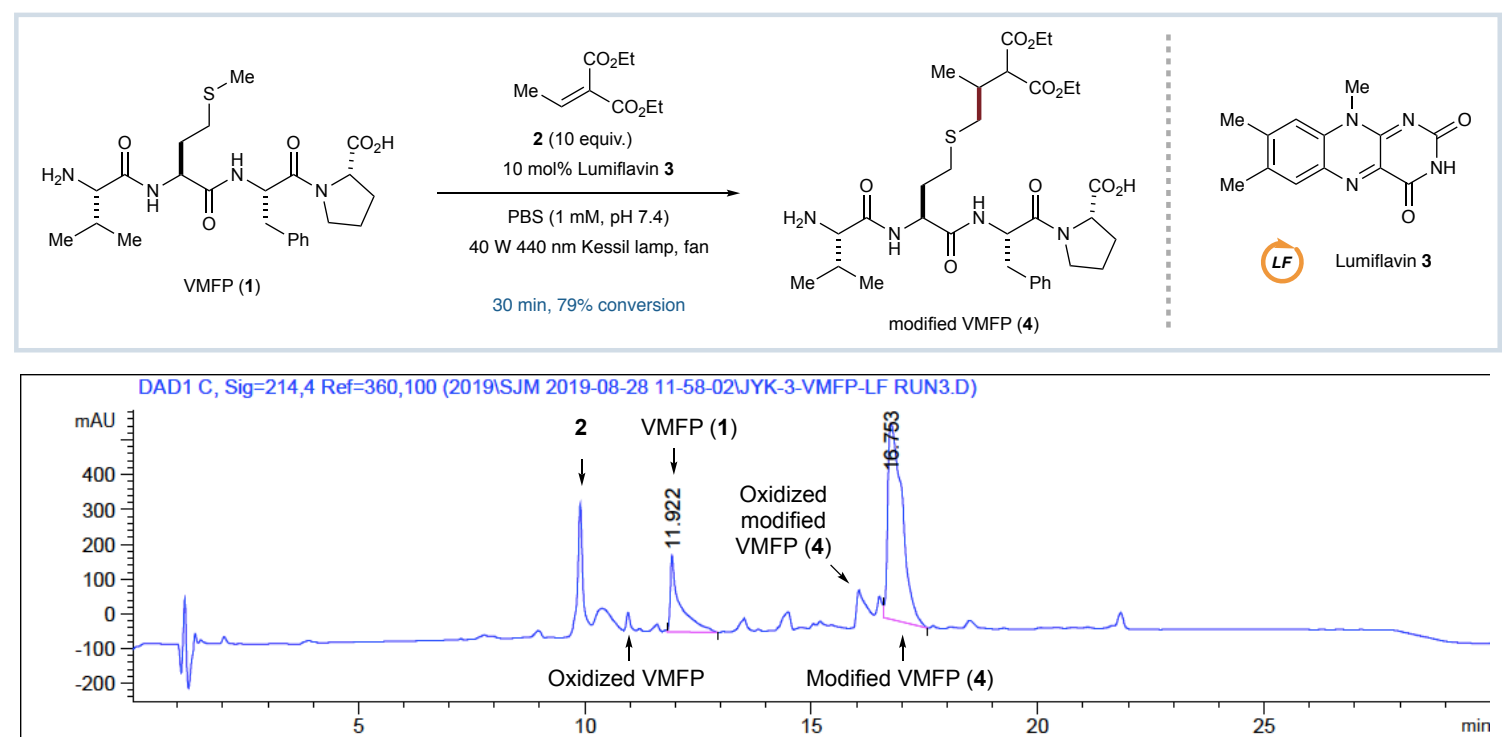

Table S2. Control experiments in the photoredox methionine-selective modification.

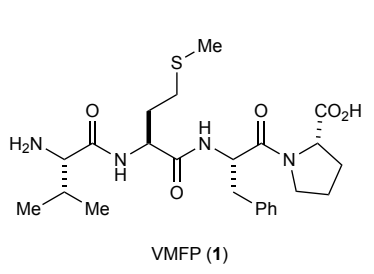

$\operatorname{VMFP}(\mathbf{1})$

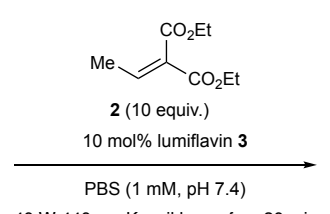

40 W 440 nm Kessil lamp, fan, 30 min

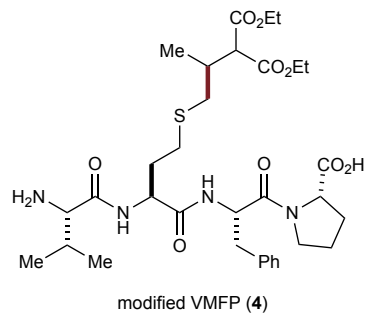

entr
1
2
3
4

conditions

Standard

no diethyl ethylidenemalonate 2

no light

no lumiflavin 3

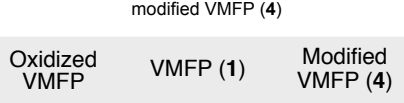

$\begin{array}{lll}- & 16 \% & 61 \%\end{array}$

\begin{tabular}{ccc}
- & $16 \%$ & $61 \%$ \\
$31 \%$ & $63 \%$ & - \\
- & $63 \%$ & - \\
\hline & $95 \%$ & -
\end{tabular}


Table S3. Amino acid residue compatibility.

\begin{tabular}{|c|c|c|c|c|}
\hline & 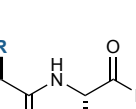 & 2 (10 equiv & $C_{13 \text { (10 equiv.) }}$ & 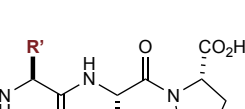 \\
\hline \multirow{2}{*}{\multicolumn{2}{|c|}{ VXFP }} & \multirow{2}{*}{\multicolumn{2}{|c|}{ PBS (1 mM, pH 7.4) }} & \multirow{2}{*}{ modified VXFP } \\
\hline & & & & \\
\hline entry & tetrapeptide & Michael acceptor & Recovered tetrapeptide & Modified tetrapeptide \\
\hline 1 & VYFP & 13 & $90 \%$ & - \\
\hline 2 & VHFP & 2 & $94 \%$ & - \\
\hline 3 & VWFP & 13 & $84 \%$ & - \\
\hline 4 & VSFP & 2 & $94 \%$ & - \\
\hline 5 & VDFP & 2 & $104 \%$ & - \\
\hline 6 & VNFP & 2 & $89 \%$ & - \\
\hline 7 & VRFP & 2 & $100 \%$ & - \\
\hline 8 & VKFP & 2 & $90 \%$ & - \\
\hline 9 & $(\mathrm{VCFP})_{2}$ & 2 & $49 \%$ & - \\
\hline
\end{tabular}

Table S4. Reactivity toward cysteine residues.

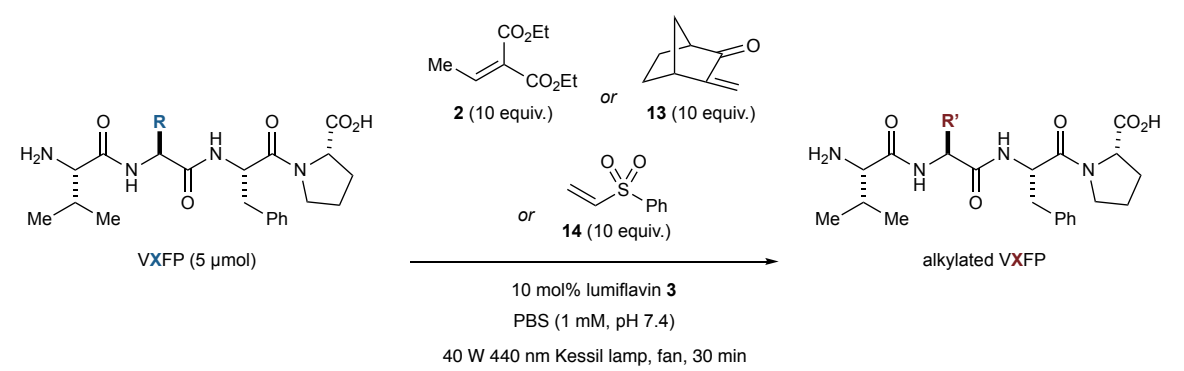

\begin{tabular}{|ccccc|}
\hline entry & tetrapeptide & Michael acceptor & Alkylated VCFP & Alkylated VMFP \\
1 & VCFP & $\mathbf{2}$ & $54 \%$ & - \\
2 & VCFP & $\mathbf{1 3}$ & $26 \%$ & - \\
3 & VCFP & $\mathbf{1 4}$ & $59 \%$ & $36 \%$ \\
4 & VCFP+VMFP & $\mathbf{2}$ & $22 \%$ & $6 \%$ \\
5 & VCFP+VMFP & $\mathbf{1 3}$ & $38 \%$ & $11 \%$ \\
6 & VCFP+VMFP & $\mathbf{1 4}$ & $75 \%$ & \\
\end{tabular}




\section{Modification of aprotinin with various Michael acceptors}

3.1. Reaction conditions and characterization methods

\subsubsection{General reaction conditions for methionine alkylation on aprotinin}

Aprotinin was purchased from G-Biosciences. Milli-Q water and DMF were degassed by the freeze-pumpthaw method (5 cycles). All stock solutions in the method were made with degassed solvents and stored in a glovebox. To a $\mathrm{V}$-shaped vial insert equipped with a stir bar was added aprotinin solution $\left(0.5 \mathrm{mM}\right.$ in $\mathrm{H}_{2} \mathrm{O}, 10 \mu \mathrm{L}, 5$

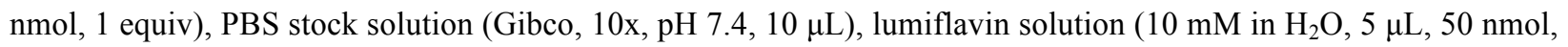
10 equiv), Michael acceptor solution (0.2 M in DMF, $5 \mu \mathrm{L}, 1 \mu \mathrm{mol}, 200$ equiv) and water (70 $\mu \mathrm{L})$. To a $2 \mathrm{~mL}$ vial was added $\mathrm{H}_{2} \mathrm{O}$ as a coolant and the vial insert containing reaction mixture. The vial was sealed and brought out of the glovebox. The reaction was stirred and irradiated using a $40 \mathrm{~W}$ Kessil blue LED lamp (PR160-440, 100\% intensity, $4 \mathrm{~cm}$ away) for $30 \mathrm{~min}$. The reaction was filtered through a centrifuge tube filter (Corning Costar, Fisher Scientific) and the filtrate was extensively washed with PBS (Gibco, 1x, pH 7.4) using a concentrator (Pierce, Thermo Fisher, 3K MWCO). Resulting aprotinin solution was analyzed by HPLC analysis or intact protein analysis.

Note: Solutions can also be degassed by sparging with nitrogen for $10 \mathrm{~min}$. Reaction mixture prepared with such degassed solvents provided protein products without significant oxidation of proteins. Also, DMSO can be utilized as an organic solvent instead of $D M F$, albeit resulting in slightly lower conversions.

Figure S2. Standard reaction setup.

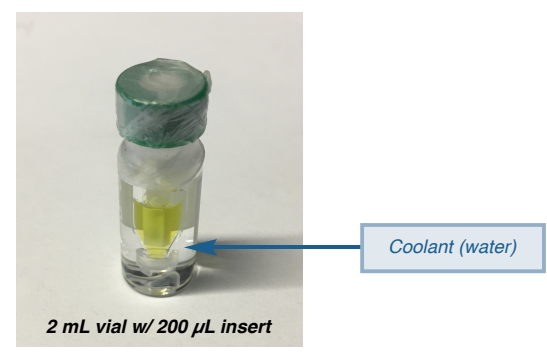

\subsubsection{Proteomics analysis}

The conversion of conjugation was characterized by HPLC analysis or intact protein analysis. The conjugation site was identified via peptide mapping strategy. HPLC analyses were performed on an Agilent 1260 Infinity LC system using a reverse-phase C18 column (Agilent Zorbax 300SB-C18, $3.5 \mu \mathrm{m}, 100 \mathrm{~mm}$ ). The eluents used were Buffer A (water + 0.1\% TFA) and Buffer B (9:1 acetonitrile: water + 0.1\% TFA). HPLC method: 1 min 100\% Buffer A, followed by 0-73\% gradient of Buffer B over 25 min, followed by 2 min flush at 90\% buffer B, followed by 2 min re-equilibration to $100 \%$ buffer A (water $+0.1 \%$ TFA). Conversion was determined by taking the ratio of $\mathrm{A}_{\text {prod }} / \mathrm{A}_{\text {total }}$ where $\mathrm{A}_{\text {prod }}=$ area in $\mathrm{mAU}$ of the product peak and $\mathrm{A}_{\text {total }}=$ area in $\mathrm{mAU}$ of the combined protein containing species (product, starting material, and byproducts).

For intact protein LC-MS, samples were prepared at $1 \mathrm{mg} / \mathrm{mL}$ in PBS and analyzed by a Waters Synapt G2 QTOF mass spectrometer connected to Aquity UPLC (Waters, Milford, MA). Samples were desalted and eluted from a reversed-phase C4 column (ACQUITY UPLC BEH C18 column 300A $1.7 \mu \mathrm{m} \mathrm{2.1x150} \mathrm{mm).}$ 
For LC/MS/MS peptide mapping analysis, samples were denatured in the presence of $0.5 \%$ Rapigest surfactant, reduced, alkylated and digested by trypsin and/or Glu-C. Digested peptides were separated on a reversedphase C18 column (ACQUITY UPLC BEH C18 column 130A 1.7um 2.1x150mm). Data was acquired on a QEPlus mass spectrometer (ThermoFisher Scientific, Waltham, MA) connected to Aquity UPLC (Waters, Milford, MA). MS was operated under positive mode with ESI voltage at $3.5 \mathrm{kV}$; sheath gas flow rate of 45 , and capillary temperature $320^{\circ} \mathrm{C}$. Sample of $1 \mu \mathrm{g}$ was injected and eluted on a Waters BEH C18 column $(2.1 \times 150 \mathrm{~mm}, 130 \AA$, $1.7 \mu \mathrm{m}$ ), heated at $50{ }^{\circ} \mathrm{C}$, using a $60 \mathrm{~min}$ LC gradient. The gradient setting was $0.2 \%$ to $40 \%$ solvent B in $30 \mathrm{~min}$ at $0.2 \mathrm{~mL} / \mathrm{min}$ flow rate.

Peptide mapping data was analyzed using Thermo BioPharma Finder software (version 2.0, ThermoFisher Scientific, Waltham, MA). The search settings were: S/N threshold, 20; mass accuracy, 5 ppm; maximum number of modifications for a peptide, 2. Cys carbamidomethylation (CAM-C) was set as the fixed modification; Met oxidation and Asn deamidation were set as the variable modifications. For alkylation searches, modifications with 2 at $186.0887 \mathrm{Da}$, mono-alkylation with $\mathbf{1 3}$ at $122.0726 \mathrm{Da}$, bis-alkylation with $\mathbf{1 3}$ at $244.1458 \mathrm{Da}$, mono-alkylation with 14 at $168.02 \mathrm{Da}$, bis-alkylation with 14 at $336.04 \mathrm{Da}$, and tri-alkylation with $\mathbf{1 4}$ at 504.6 Da were set as the variable modification on Met accordingly. MS results were manually verified. 


\subsection{Michael acceptor substrates}

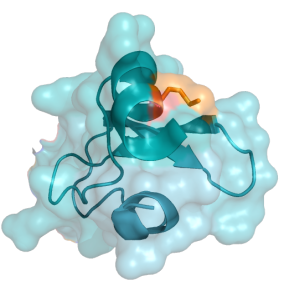

Aprotinin (M.W. = 6511)

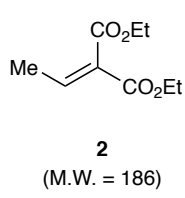

$93 \%$ conversion mono-alkylation

Aprotinin reacted with 2 under the aforementioned general reaction conditions. Conversion (93\%) was determined based on HPLC analysis. LC-MS/MS indicated the reaction was selective exclusively for Met 52.
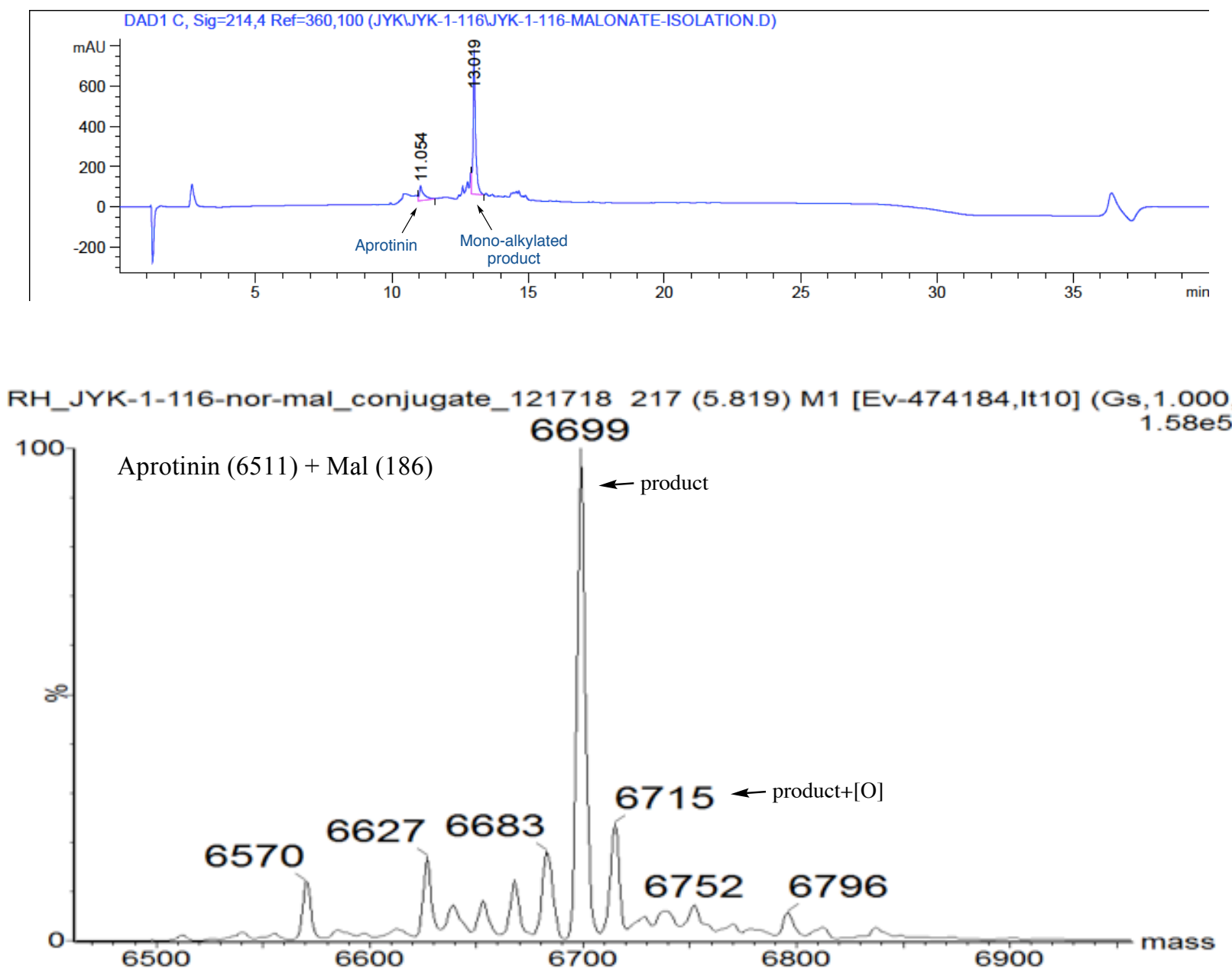


\begin{tabular}{|ccccc|}
$\mathbf{b}^{+}$ & \multicolumn{3}{c}{ Sequence } & $\mathbf{y}^{+}$ \\
88.0394 & 1 & $\mathbf{S}$ & 7 & - \\
159.0764 & 2 & $\mathbf{A}$ & 6 & 967.3860 \\
288.1191 & 3 & $\mathbf{E}$ & 5 & 896.3489 \\
403.1460 & 4 & $\mathbf{D}$ & 4 & 767.3063 \\
563.1766 & 5 & CAM-C & 3 & 652.2793 \\
880.3063 & 6 & $\mathbf{2 +} \mathbf{M}^{52}$ & 2 & 492.2487 \\
- & 7 & $\mathbf{R}$ & 1 & 175.1190 \\
\hline
\end{tabular}

File: RH_PeptideConjugation_JYK-1-116-nor-mal_12182018

F: FTMS + c ESI d Full ms2 527.7128@hcd27.00 [733.0000-1095.0000]

TIC: $1.31 \mathrm{e}+007$ Experimental $(+2, w=2, r=17500$, QExactivePlus)

S-A-E-D-CAM-C- $(2+\mathbf{M})-\mathrm{R}$

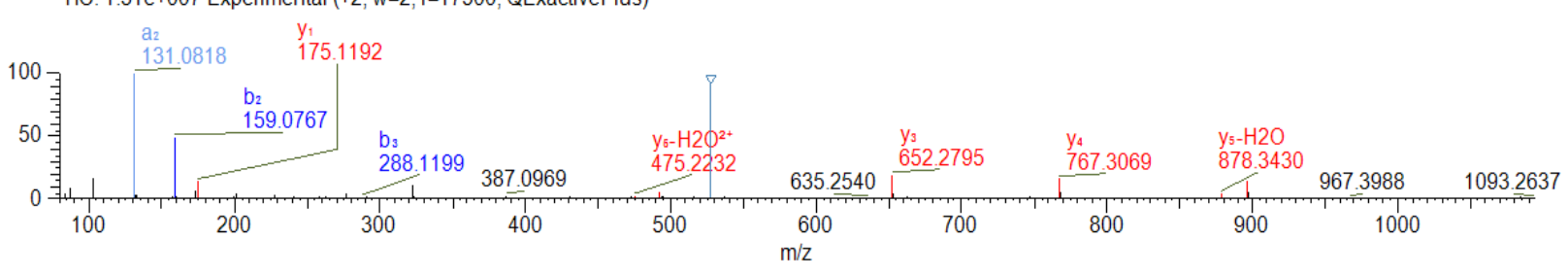



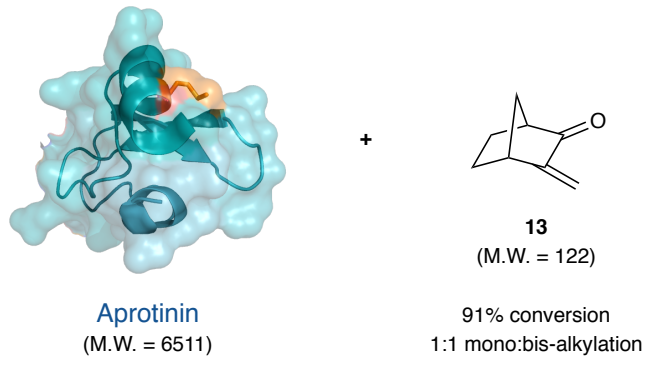

Aprotinin reacted with $\mathbf{1 3}$ under the aforementioned general reaction conditions. Conversion (91\%) was determined based on HPLC analysis. LC-MS/MS indicated the reaction was selective exclusively for Met 52.

DAD1 C, Sig=214,4 Ref=360,100 (JYKUYK-1-116WYK-1-116-NORBORNANONE-ISOLATION.D)
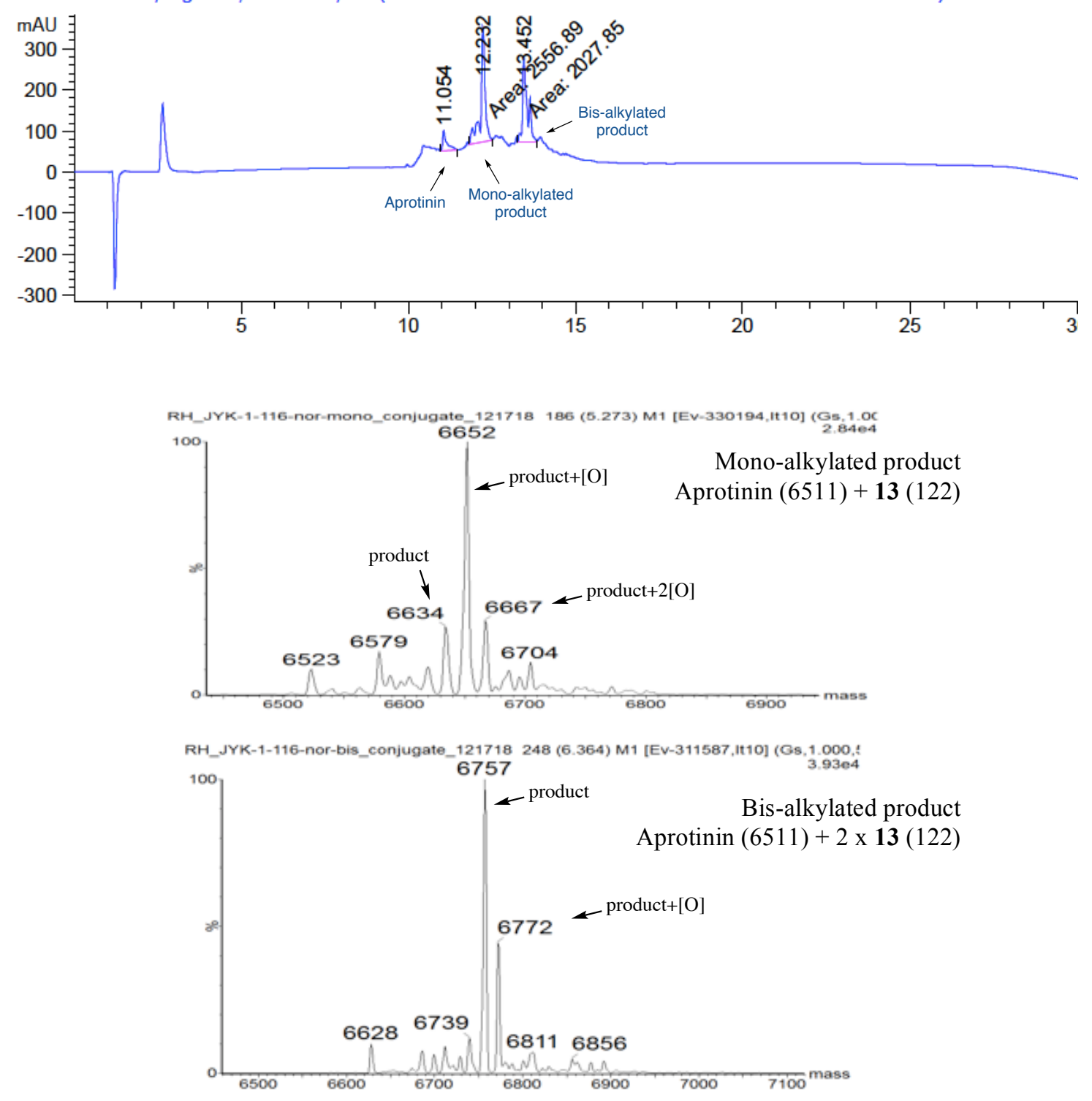


\begin{tabular}{|ccccc|}
$\mathbf{b}^{+}$ & \multicolumn{3}{c}{ Sequence } & $\mathbf{y}^{+}$ \\
\hline 88.0394 & 1 & $\mathbf{S}$ & 7 & - \\
159.0764 & 2 & $\mathbf{A}$ & 6 & 903.3693 \\
288.1190 & 3 & $\mathbf{E}$ & 5 & $\mathbf{8 3 2 . 3 3 2 2}$ \\
403.1460 & 4 & $\mathbf{D}$ & 4 & $\mathbf{7 0 3 . 2 8 9 6}$ \\
563.1766 & 5 & CAM-C & 3 & $\mathbf{5 8 8 . 2 6 2 7}$ \\
816.2897 & 6 & $\mathbf{1 3}+\mathbf{M}^{52}$ & 2 & 428.232 \\
- & 7 & $\mathbf{R}$ & 1 & $\mathbf{1 7 5 . 1 1 9}$ \\
\hline
\end{tabular}

File: RH_PeptıdeConjugatıon_JYK-1-116-nor-mono_12182018

F: FTMS + c ESI d Full ms2 495 7049@hcd27 00 [68-6667-1030.0000] TIC: $2.04 \mathrm{e}+005$ Experimental ( $+2, w=2, r=17500$, QExactivePlus)

S-A-E-D-CAM-C-(13+M)-R
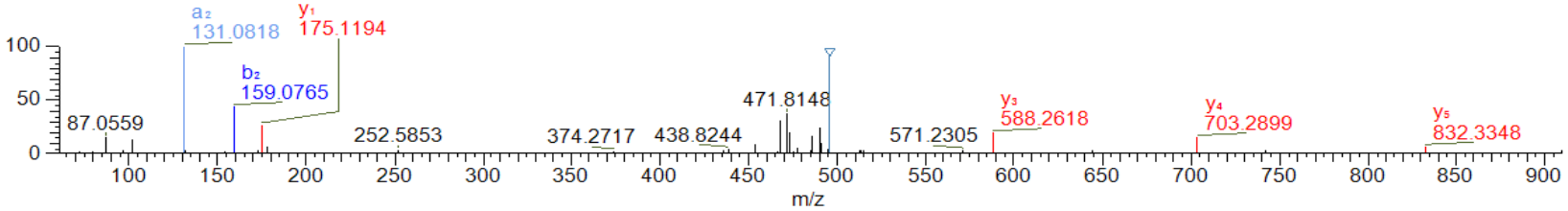

\begin{tabular}{|c|}
\hline $\mathbf{b}^{+}$ \\
\hline 88.0394 \\
\hline 159.0764 \\
\hline 288.1191 \\
\hline 403.1460 \\
\hline 563.1766 \\
\hline 938.3629 \\
\hline- \\
\hline
\end{tabular}

\begin{tabular}{ccc} 
Sequence & \multicolumn{1}{c}{$\mathbf{y}^{+}$} \\
$\mathbf{S}$ & 7 & - \\
$\mathbf{A}$ & 6 & 1025.4425 \\
$\mathbf{E}$ & 5 & 954.4054 \\
$\mathbf{D}$ & 4 & $\mathbf{8 2 5 . 3 6 2 8}$ \\
CAM-C & 3 & 710.3359 \\
$2 \mathrm{x} \mathbf{1 3}+\mathbf{M}^{52}$ & 2 & $\mathbf{5 5 0 . 3 0 5 2}$ \\
$\mathbf{R}$ & 1 & $\mathbf{1 7 5 . 1 1 9}$
\end{tabular}

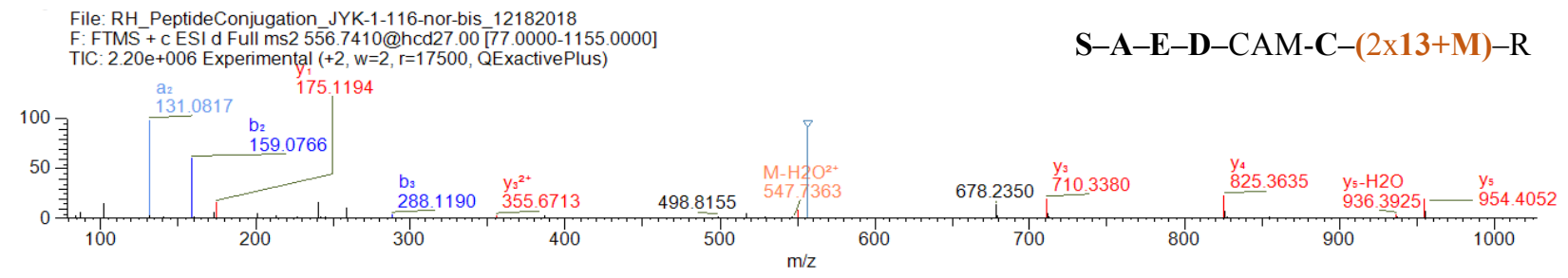



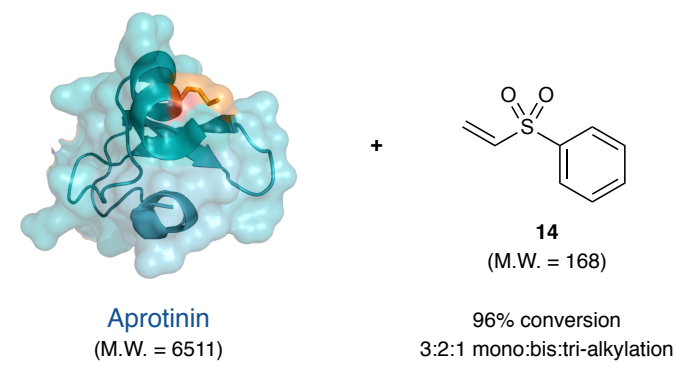

Aprotinin reacted with 14 under the aforementioned general reaction conditions. Conversion (96\%) was determined based on HPLC analysis. LC-MS/MS indicated the reaction was selective exclusively for Met 52.

DAD1 C, Sig=214,4 Ref=360,100 (JYKLYK-1-095WYK-1-095-APRO-LUMI-30MIN-PBS.D)
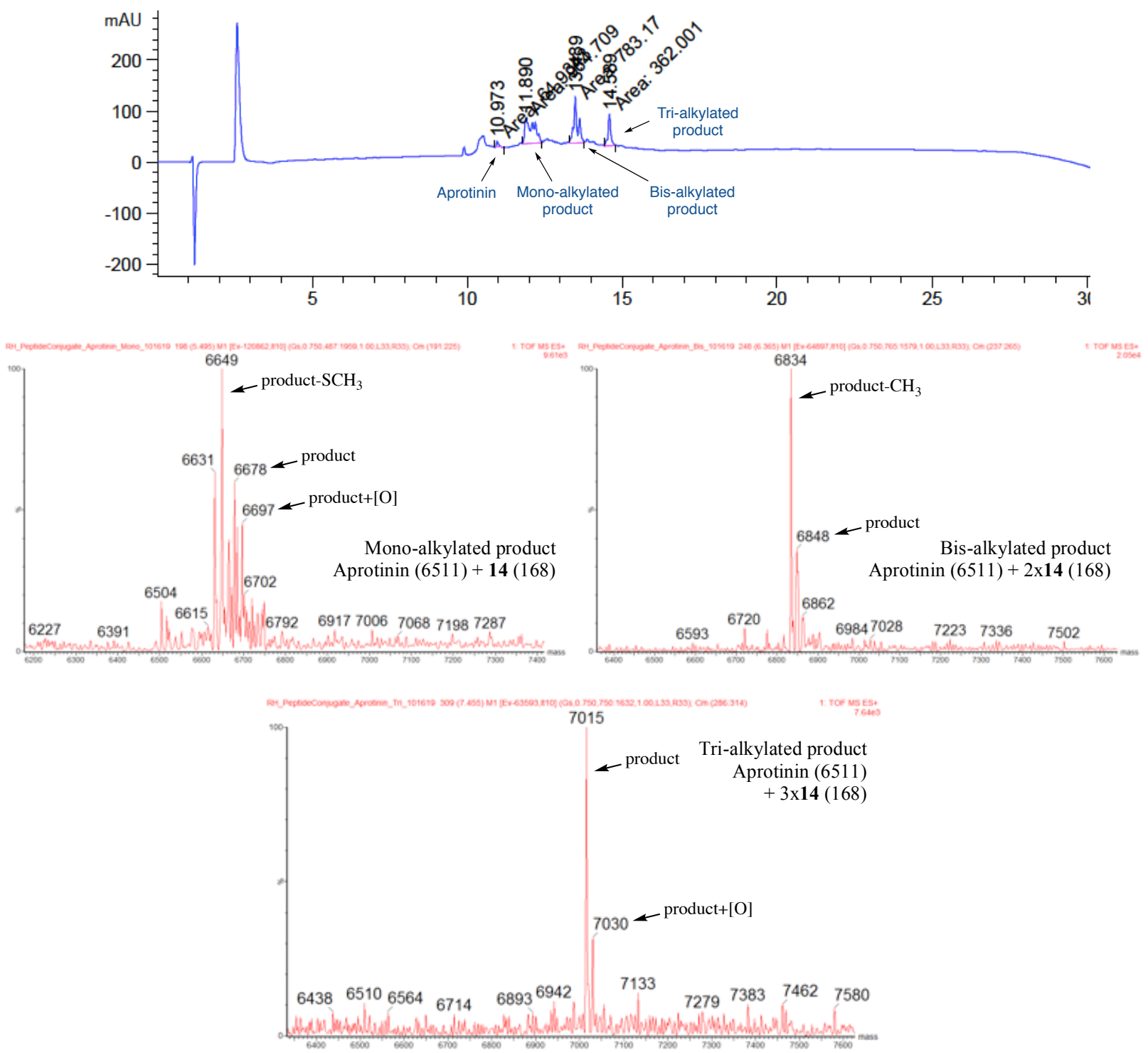


\begin{tabular}{|c|c|}
\hline $\mathbf{b}^{+}$ & \\
\hline 88.0394 & 1 \\
\hline 159.0764 & 2 \\
288.1190 & 3 \\
403.1460 & 4 \\
563.1766 & 5 \\
816.2897 & 6 \\
- & 7 \\
\hline
\end{tabular}

\begin{tabular}{ccc} 
Sequence & & $\mathbf{y}^{\mathbf{+}}$ \\
$\mathbf{S}$ & 7 & - \\
$\mathbf{A}$ & 6 & 949.3213 \\
$\mathbf{E}$ & 5 & $\mathbf{8 7 8 . 2 8 4 2}$ \\
$\mathbf{D}$ & 4 & 749.2416 \\
$\mathrm{CAM}^{\mathbf{C}}$ & 3 & $\mathbf{6 3 4 . 2 1 4 7}$ \\
$\mathbf{1 4}+\mathbf{M}^{52}$ & 2 & 474.184 \\
$\mathbf{R}$ & 1 & $\mathbf{1 7 5 . 1 1 9}$ \\
\hline
\end{tabular}

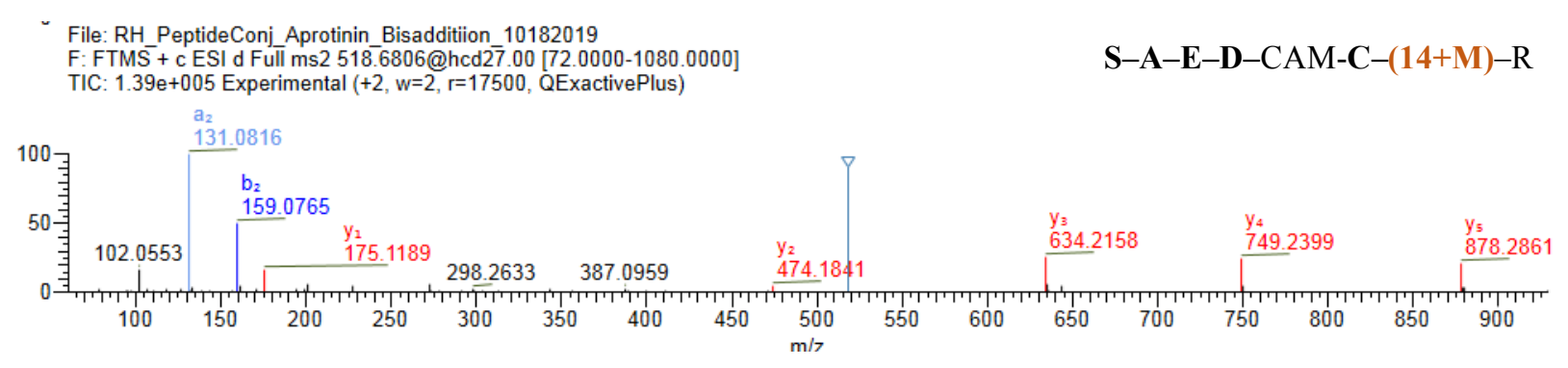

\begin{tabular}{c|c}
$\mathbf{b}^{+}$ & \\
\hline 88.0394 & 1 \\
159.0764 & 2 \\
288.1191 & 3 \\
403.1460 & 4 \\
563.1766 & 5 \\
1030.2662 & 6 \\
- & 7 \\
\hline
\end{tabular}

\begin{tabular}{ccc} 
Sequence & & $\mathbf{y}^{+}$ \\
$\mathbf{S}$ & 7 & - \\
$\mathbf{A}$ & 6 & 1117.3458 \\
$\mathbf{E}$ & 5 & $\mathbf{1 0 4 6 . 3 0 8 7}$ \\
$\mathbf{D}$ & 4 & 917.2661 \\
$\mathrm{CAM}-\mathbf{C}$ & 3 & $\mathbf{8 0 2 . 2 3 9 2}$ \\
$2 \times \mathbf{1 4}+\mathbf{M}^{52}$ & 2 & $\mathbf{6 4 2 . 2 0 8 5}$ \\
$\mathbf{R}$ & 1 & $\mathbf{1 7 5 . 1 1 9}$ \\
\hline
\end{tabular}

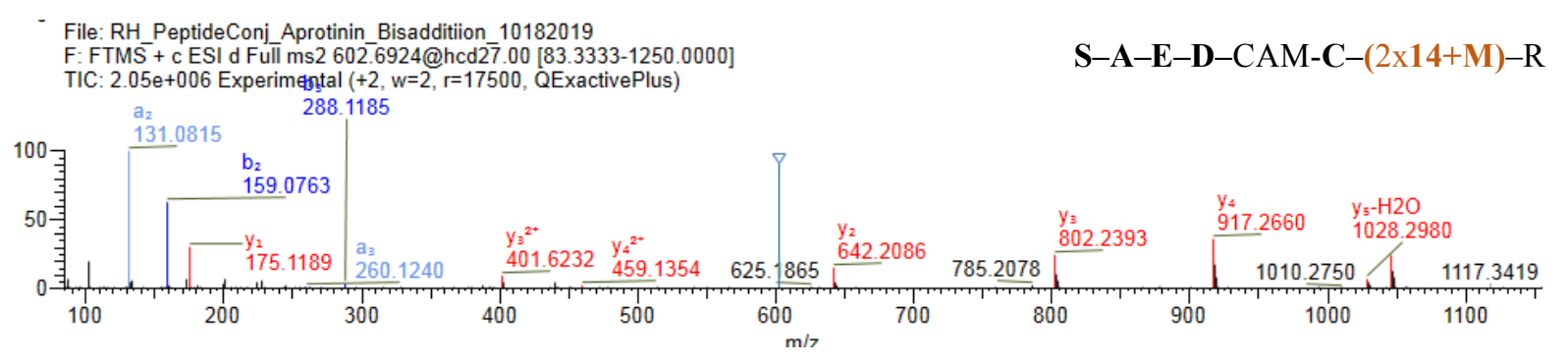




\begin{tabular}{|ccccc|}
$\mathbf{b}^{+}$ & \multicolumn{3}{c}{ Sequence } & $\mathbf{y}^{+}$ \\
\hline 88.0394 & 1 & $\mathbf{S}$ & 7 & - \\
159.0764 & 2 & $\mathbf{A}$ & 6 & $1285.3703 \mathrm{c}$ \\
288.1191 & 3 & $\mathbf{E}$ & 5 & $\mathbf{1 2 1 4 . 3 3 3 2}$ \\
403.1460 & 4 & $\mathbf{D}$ & 4 & $\mathbf{1 0 8 5 . 2 9 0 6}$ \\
563.1766 & 5 & CAM-C & 3 & 970.2637 \\
1198.2907 & 6 & $3 \times \mathbf{1 4}+\mathbf{M}^{52}$ & 2 & $\mathbf{8 1 0 . 2 3 3}$ \\
- & 7 & $\mathbf{R}$ & 1 & 175.119 \\
\hline
\end{tabular}

- File: RH_PeptideConj_Aprotinin_Triaddition_10222019

F: FTMS + c ESI d Full ms2 686.7053@hcd27.00 [94.6667-1420.0000]

TIC: $9.24 \mathrm{e}+005$ Experimental $(+2, w=2, r=17500$, QExactivePlus)

S-A-E-D-CAM-C- $(3 \times 14+M)-R^{n}{ }^{n}$

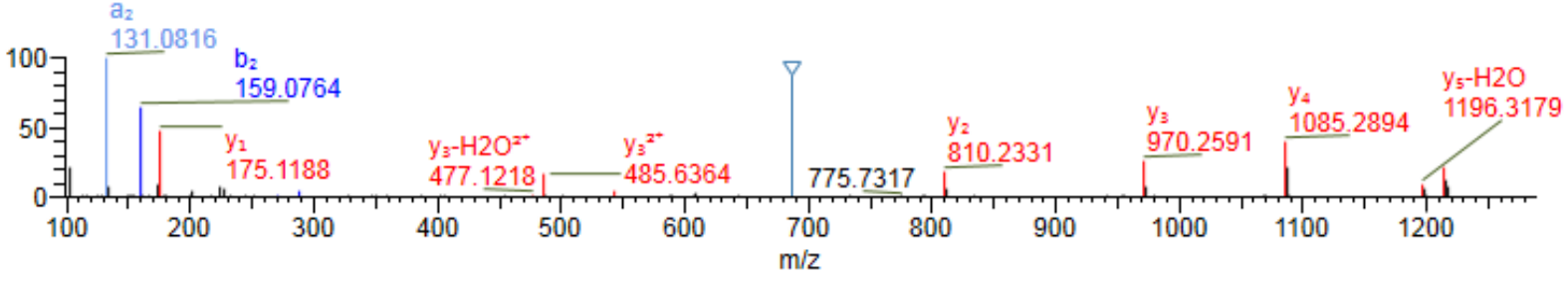




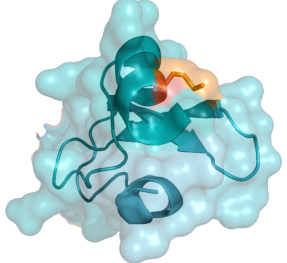

Aprotinin (M.W. $=6511)$

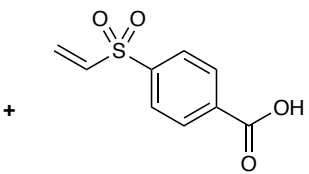

15

(M.W. = 210)

$>95 \%$ conversion 3:4:1 mono:bis:tri-alkylation

Aprotinin reacted with 15 under the aforementioned general reaction conditions. Full conversion was determined based on HPLC analysis. LC-MS indicated formation of mono-, bis- and tri-alkylated products.
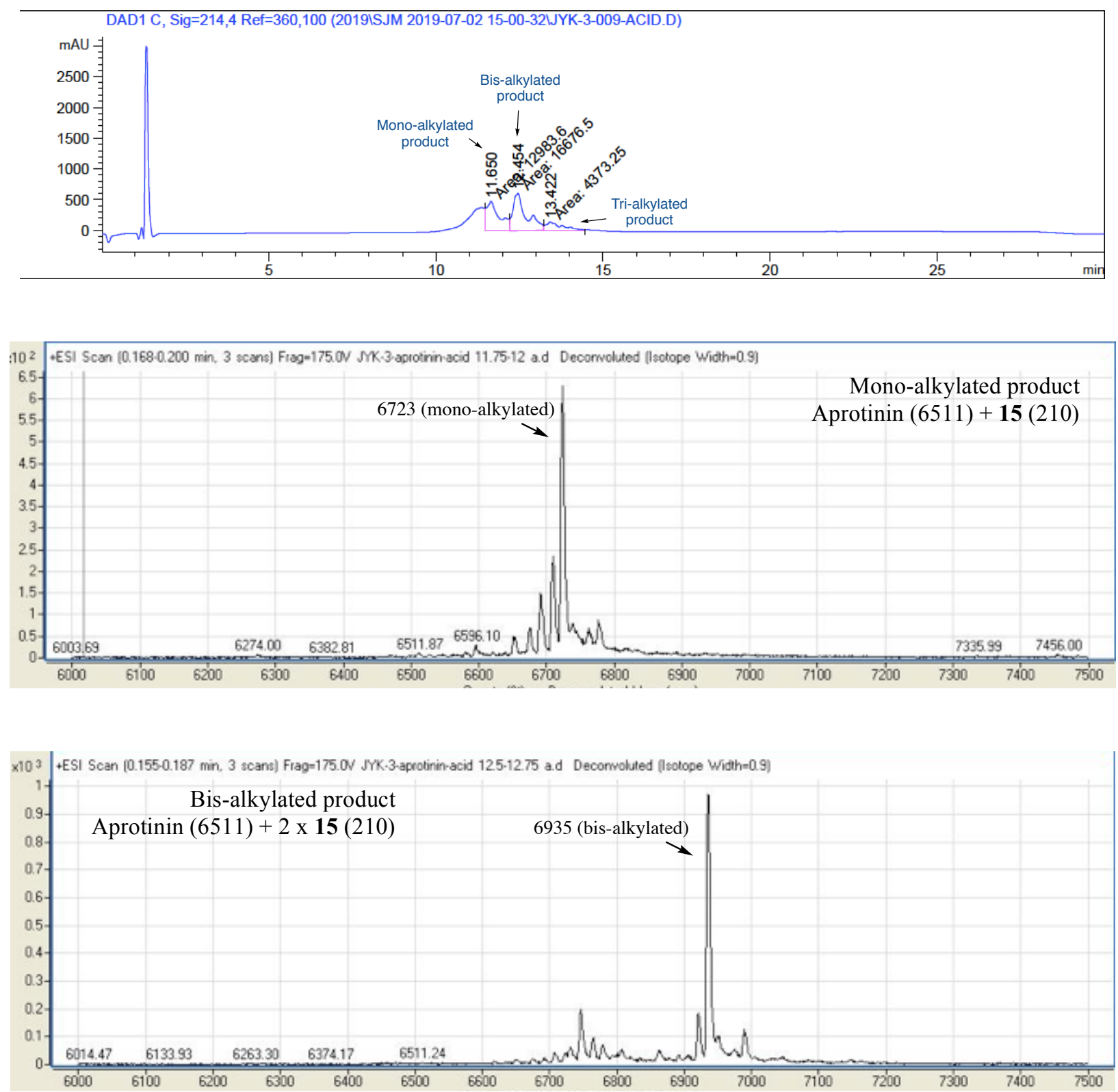


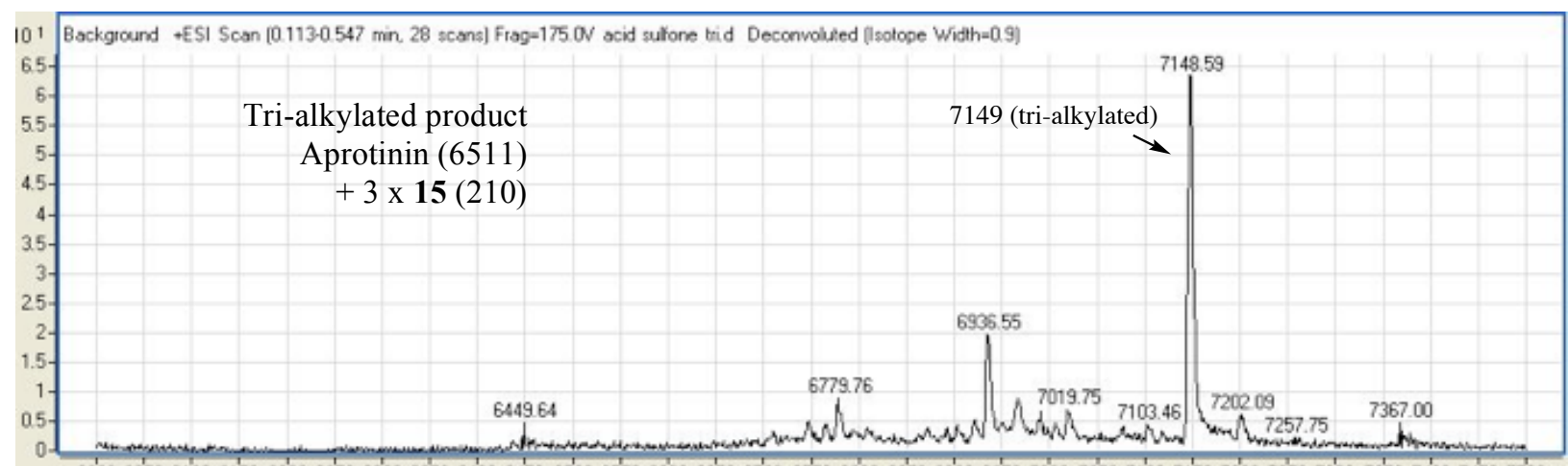
$6000605061006150620062506300635064006450650065506600665067006750660068506900695070007050710071507200725073 \dot{1} 007350740074507500$ 

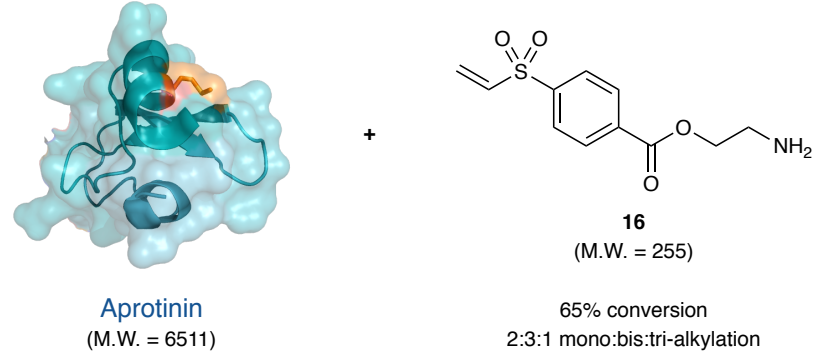

Aprotinin reacted with $\mathbf{1 6}$ under the aforementioned general reaction conditions. Modified products were not separable on HPLC analysis. Alkylation ratio was determined by intact mass analysis.

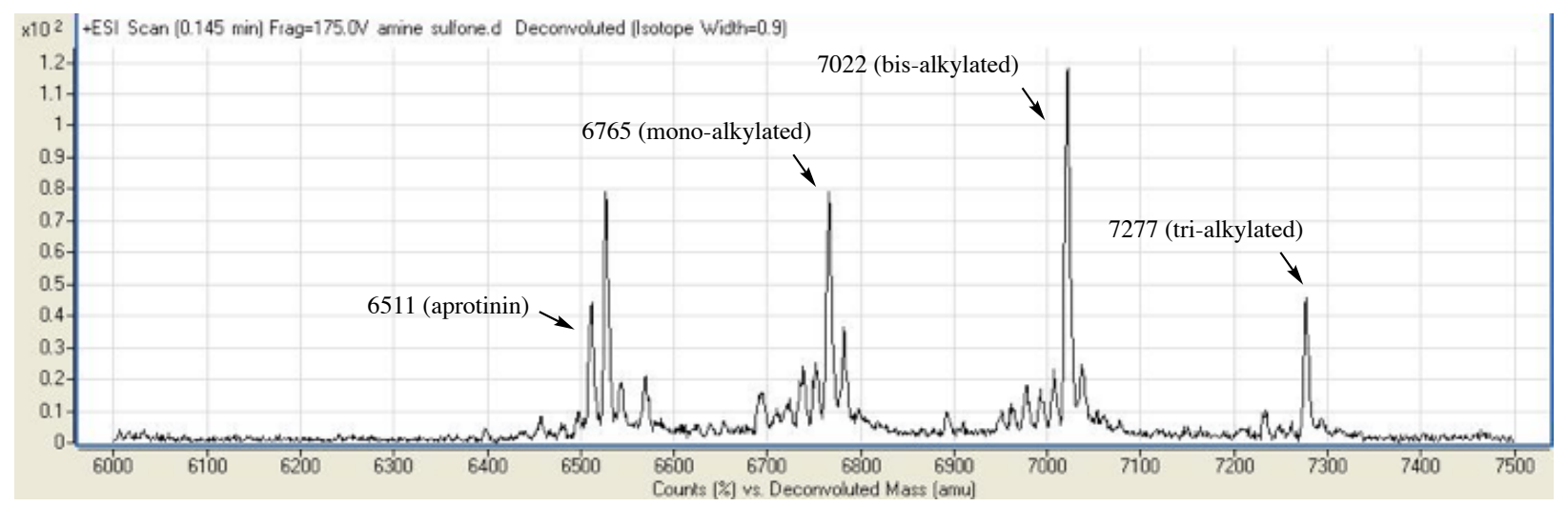




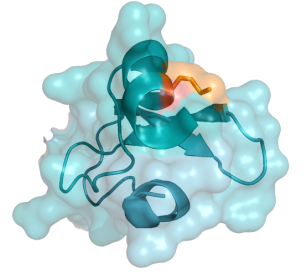

Aprotinin (M.W. $=6511)$

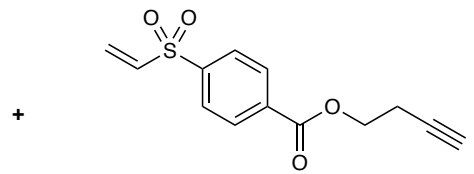

17

(M.W. $=264)$

$>95 \%$ conversion

2:1 mono:bis-alkylation

Aprotinin reacted with 17 under the aforementioned general reaction conditions. Full conversion was determined based on HPLC analysis. LC-MS indicated formation of mono-, bis- and tri-alkylated products.
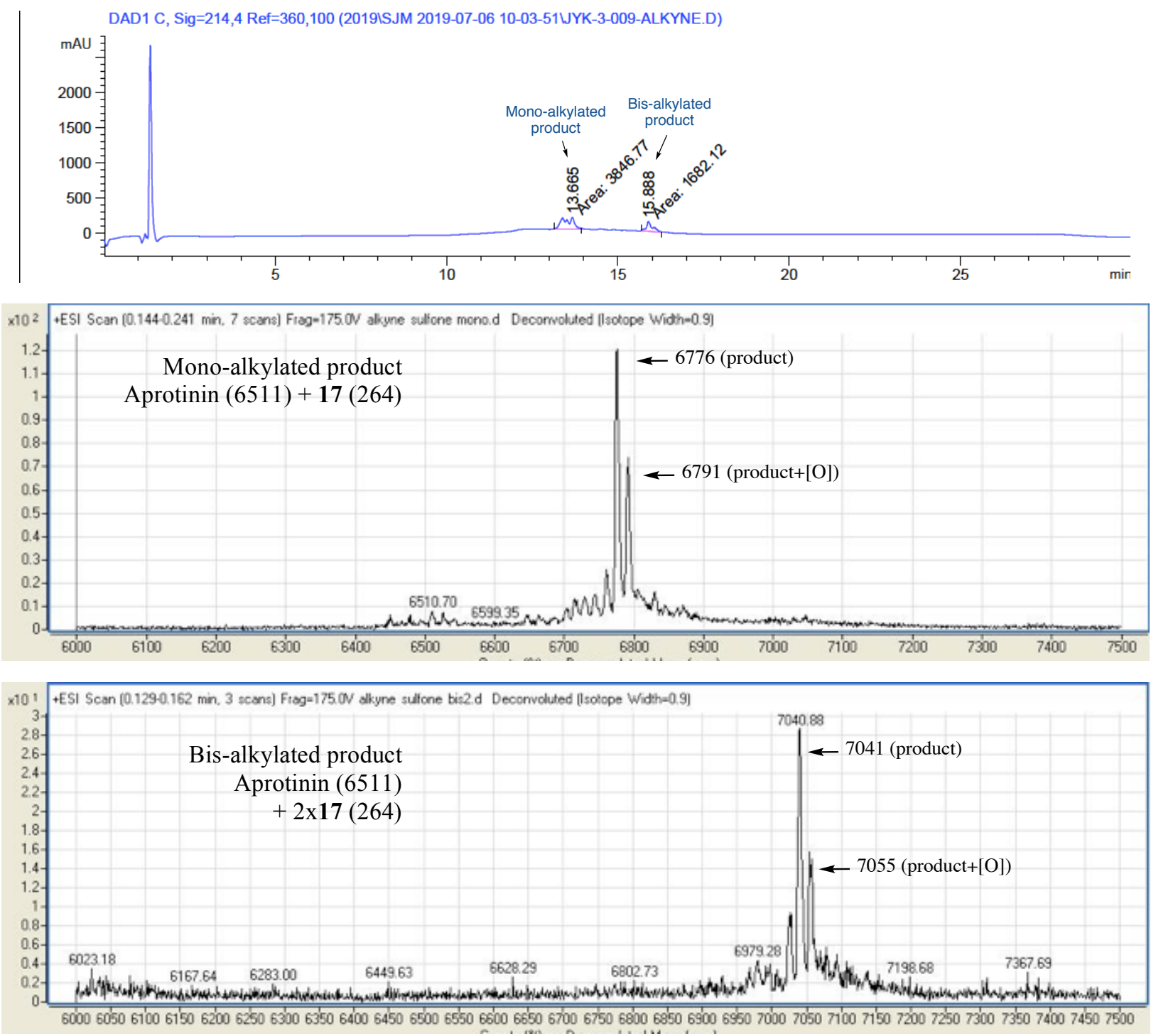


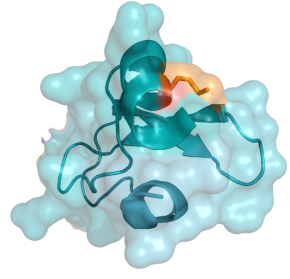

Aprotinin (M.W. $=6511)$

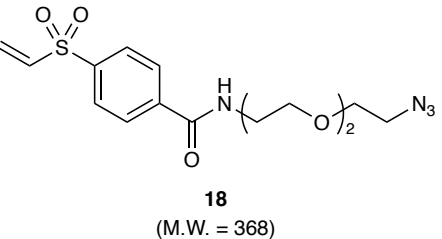

$80 \%$ conversion mono-alkylation

Aprotinin reacted with $\mathbf{1 8}$ under the aforementioned general reaction conditions. Modified products were not separable on HPLC analysis. Conversion was determined by intact mass analysis.

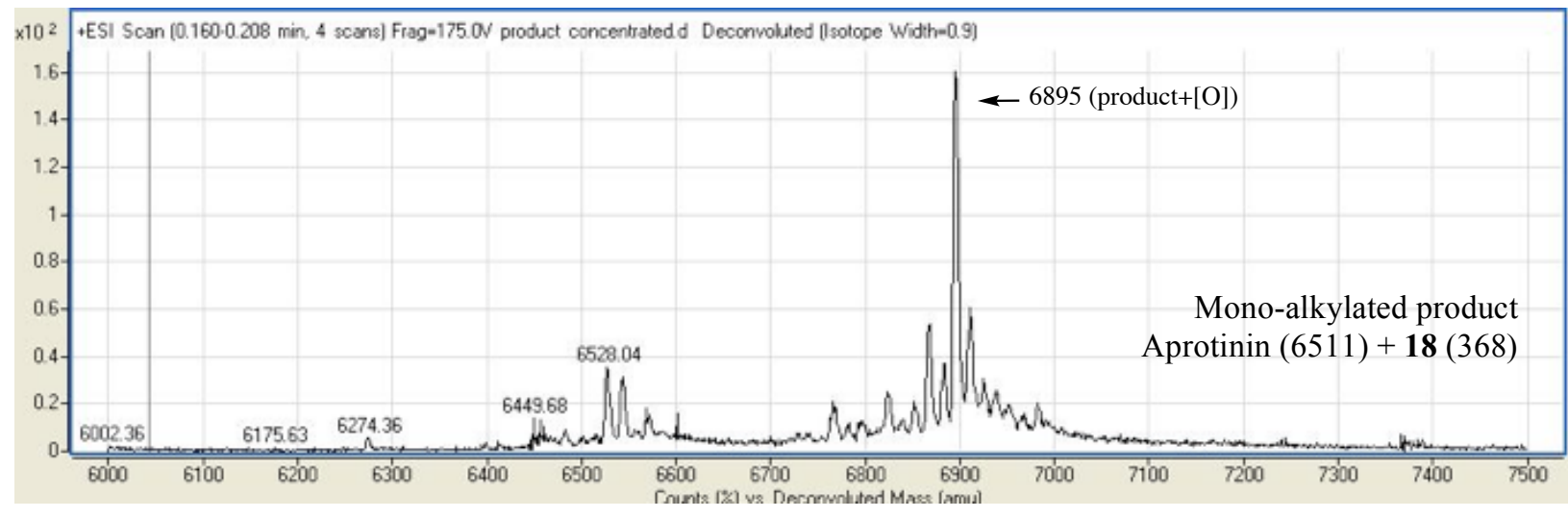




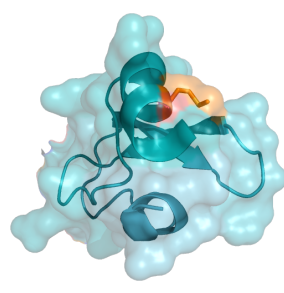

Aprotinin (M.W. = 6511)

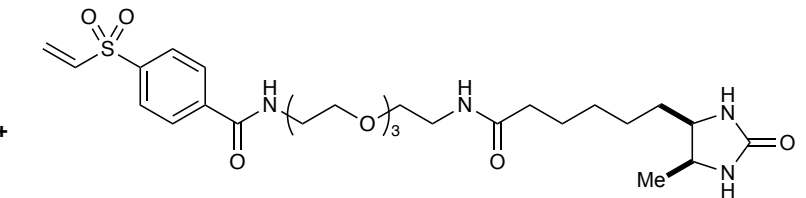

19

(M.W. = 582)

$48 \%$ conversion

mono-alkylation

Aprotinin reacted with 19 under the aforementioned general reaction conditions. Conversion (48\%) was determined based on HPLC analysis.
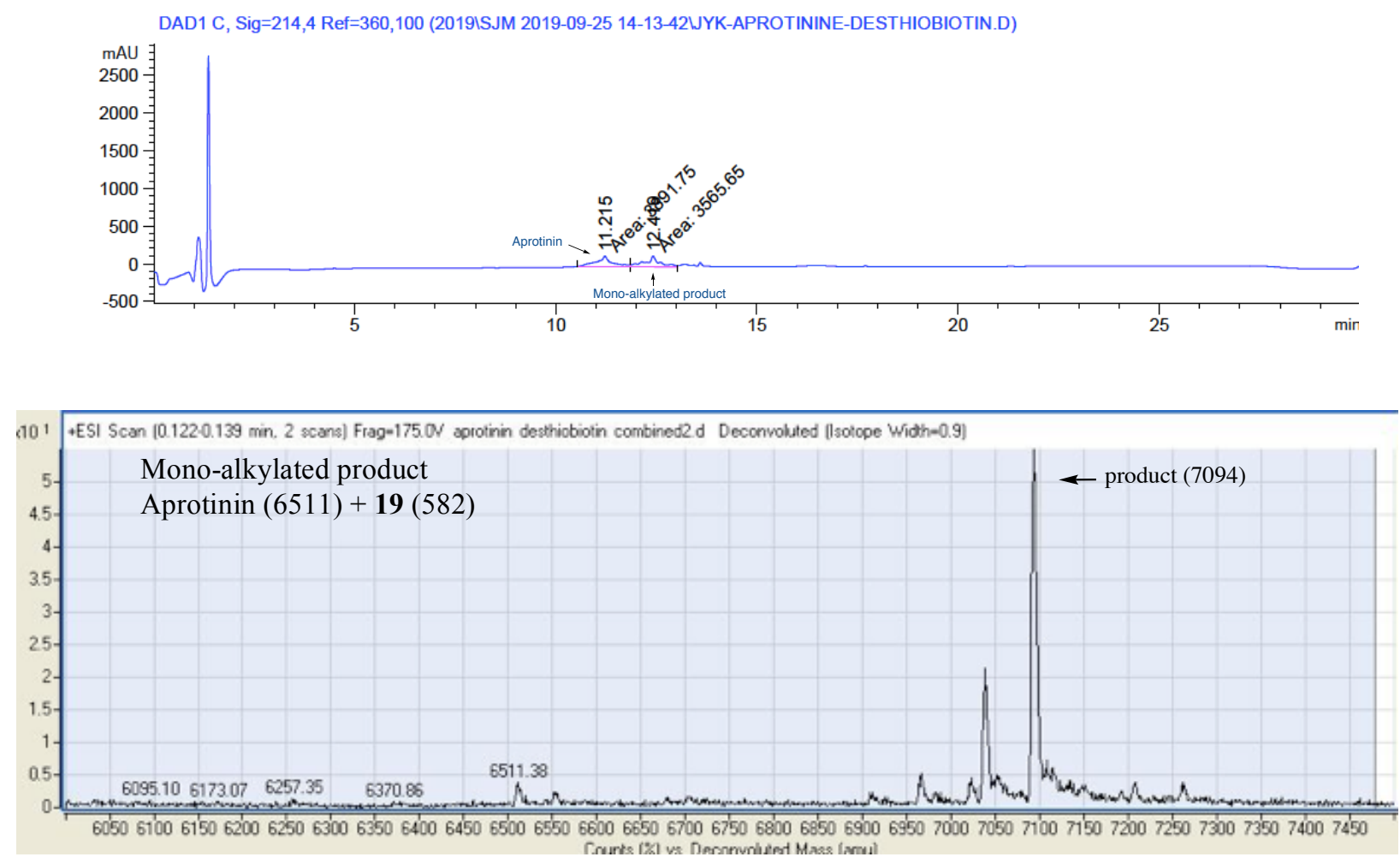


\section{Protein scope and characterization}

\subsection{Reaction conditions and characterization methods}

\subsubsection{General reaction conditions for methionine alkylation on protein substrates}

Milli-Q water and DMF were degassed by the freeze-pump-thaw method (5 cycles). All stock solutions were prepared with degassed solvents and stored in a glovebox. To a V-shaped vial insert (VWR, $200 \mu \mathrm{L})$ equipped with a stir bar was added protein solution $\left(0.5 \mathrm{mM}\right.$ in $\left.\mathrm{H}_{2} \mathrm{O}, 10 \mu \mathrm{L}, 5 \mathrm{nmol}\right)$, PBS stock solution (Gibco, 10x, pH 7.4, $10 \mu \mathrm{L})$, lumiflavin solution (10 $\mathrm{mM}$ in $\left.\mathrm{H}_{2} \mathrm{O}, 5 \mu \mathrm{L}, 50 \mathrm{nmol}\right)$, diethyl ethylidenemalonate solution (2, $0.2 \mathrm{M}$ in DMF, $5 \mu \mathrm{L}, 1 \mu \mathrm{mol})$ and water $(70 \mu \mathrm{L})$. To a $2 \mathrm{~mL}$ vial was added $\mathrm{H}_{2} \mathrm{O}$ as a coolant and the vial insert containing reaction mixture. The vial was sealed and brought out of the glovebox. The reaction was stirred and irradiated using a $40 \mathrm{~W}$ Kessil blue LED lamp (PR160-440, 100\% intensity, $4 \mathrm{~cm}$ away) for $30 \mathrm{~min}$. The reaction was filtered through centrifuge tube filters (Corning Costar, Fisher Scientific) and the filtrate was purified by spin desalting columns (Zeba, Thermo Fisher, $0.5 \mathrm{~mL}$. 7K MWCO). Purified product was subjected to LC-MS for intact mass analysis. The products were digested via trypsin and subjected to LC-MS/MS to determine the selectivity among methionine residues.

Note: Solutions can also be degassed by sparging with nitrogen for $10 \mathrm{~min}$. Reaction mixture prepared with such degassed solvents provided protein products without significant oxidation of proteins. Also, DMSO can be utilized as an organic solvent instead of DMF, albeit resulting in slightly lower conversions.

\subsubsection{Proteomics analysis}

The conversion of conjugation was characterized at the intact protein analysis and the conjugation site was identified via peptide mapping strategy. For intact protein LC-MS, samples were prepared at $1 \mathrm{mg} / \mathrm{mL}$ in PBS and analyzed by a Waters Synapt G2 QTOF mass spectrometer connected to Aquity UPLC (Waters, Milford, MA). Samples were desalted and eluted from a reversed-phase C4 column (ACQUITY UPLC BEH C18 column 300A 1.7um 2.1x150mm).

For LC-MS/MS peptide mapping analysis, samples were denatured in the presence of $0.5 \%$ Rapigest surfactant, reduced, alkylated and digested by trypsin. Digested peptides were separated on a reversed-phase C18 column (ACQUITY UPLC BEH C18 column 130A 1.7um 2.1x150mm). Data was acquired on a QEPlus mass spectrometer (ThermoFisher Scientific, Waltham, MA) connected to Aquity UPLC (Waters, Milford, MA), and analyzed using Thermo BioPharma Finder software (ThermoFisher Scientific, Waltham, MA). MS results were manually verified. Selectivity among methionine residues was determined by comparing ion counts of peptides containing modified methionines. Predicted $\boldsymbol{b}$ and $\boldsymbol{y}$ ions are tabulated below each substrate. Observed $\boldsymbol{b}$ and $\boldsymbol{y}$ ions are highlighted in blue. Diethyl ethylidenemalonate modification on methionine (Mal-M) results in +186.0892 in mass. Carbamidomethylation of cysteine (CAM-C) is an artifact due to trypsin digestion during proteomics sample preparation.

Peptide mapping data was analyzed using Thermo BioPharma Finder software (version 2.0, ThermoFisher Scientific, Waltham, MA). For protein database, UniProt IDs of proteins are as follow: uqibuitin (P0CG48), 
lactalbumin (P00711), myoglobin (P68082), carbonic anhydrase (P00921), ribonucelase A (P61823). Sequences for recombinant hGH and recombinant EGFP was obtained from vendor websites (Genscript and Chromotek, respectively). The search settings were: $\mathrm{S} / \mathrm{N}$ threshold, 20; mass accuracy, $5 \mathrm{ppm}$; maximum number of modifications for a peptide, 2. Cys carbamidomethylation (CAM-C) was set as the fixed modification; Met oxidation and Asn deamidation were set as the variable modifications. For alkylation searches, modification with 2 at 186.0887 Da was set as the variable modification on Met and Cys accordingly. MS results were manually verified. 


\subsection{Protein substrates}

\subsubsection{Ubiquitin}

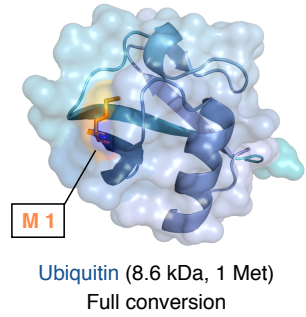

Ubiquitin (20, PDB ID 1UBQ) reacted with diethyl ethylidenemalonate (2) under the aforementioned general reaction conditions. The reaction mixture was irradiated for 90 minutes, and then purified with a Zeba spin desalting column (Thermo Scientific, $0.5 \mathrm{~mL}, 7 \mathrm{kDa}$ ) to remove any small molecule reaction components. Conversion (>90\%) was determined based on intact protein mass analysis. LC-MS/MS indicated the reaction was selective exclusively for Met 1 . The full protein sequence is shown below, with the relevant peptide highlighted.

\begin{tabular}{|c|c|c|c|c|c|}
\hline${ }^{1} \mathrm{M}$ QIFVKTLTG & KTITLEVEPS & DTIENVKAKI & QDKEGIPPDQ & QRLIFAGKQL & EDGRTLSDYN \\
\hline IQKESTLHLV & LRLRGG $^{76}$ & & & & \\
\hline
\end{tabular}
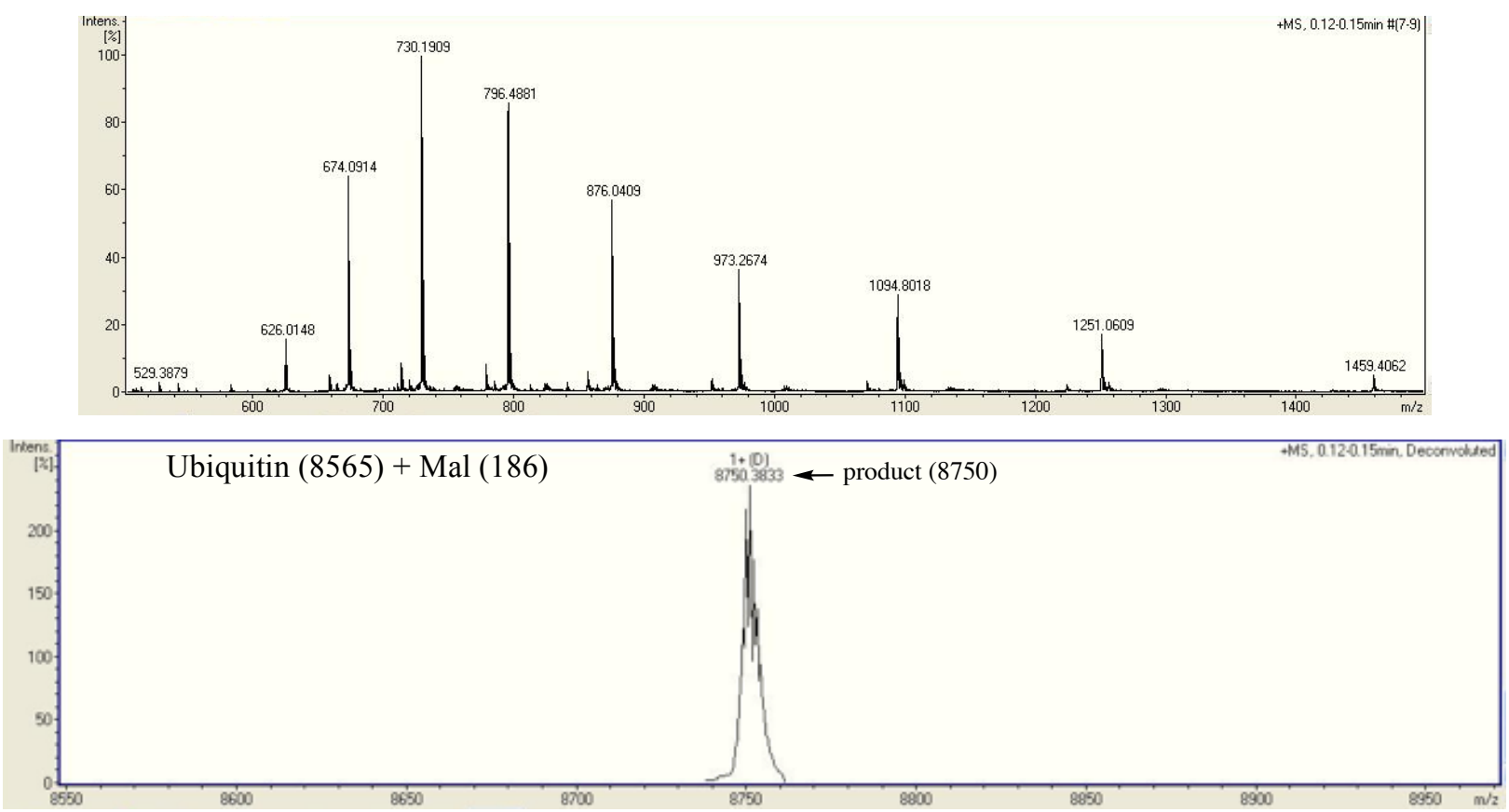


\begin{tabular}{|ccccccc|}
$\mathbf{b}^{+}$ & $\mathbf{b}^{\mathbf{+}}$ & & Sequence & $\mathbf{y}^{\mathbf{+}}$ & $\mathbf{y}^{\mathbf{+}}$ \\
\hline $\mathbf{3 1 8 . 1 3 6 9 6}$ & 159.57212 & 1 & Mal-M & 6 & & 317.69977 \\
$\mathbf{4 4 6 . 1 9 5 5 4}$ & 223.60141 & 2 & $\mathbf{Q}$ & 5 & $\mathbf{6 3 4 . 3 9 2 2 7}$ & 253.67049 \\
$\mathbf{5 5 9 . 2 7 9 6 0}$ & 280.14344 & 3 & $\mathbf{I}$ & 4 & $\mathbf{5 0 6 . 3 3 3 7 0}$ & $\mathbf{1 9 7 . 1 2 8 4 5}$ \\
706.34802 & 353.67765 & 4 & $\mathbf{F}$ & 3 & $\mathbf{3 9 3 . 2 4 9 6 3}$ & 123.59425 \\
805.41643 & 403.21185 & 5 & $\mathbf{V}$ & 2 & $\mathbf{2 4 6 . 1 8 1 2 2}$ & 74.06004 \\
\hline & - & 6 & $\mathbf{K}$ & 1 & $\mathbf{1 4 7 . 1 1 2 8 0}$ & \\
\hline
\end{tabular}

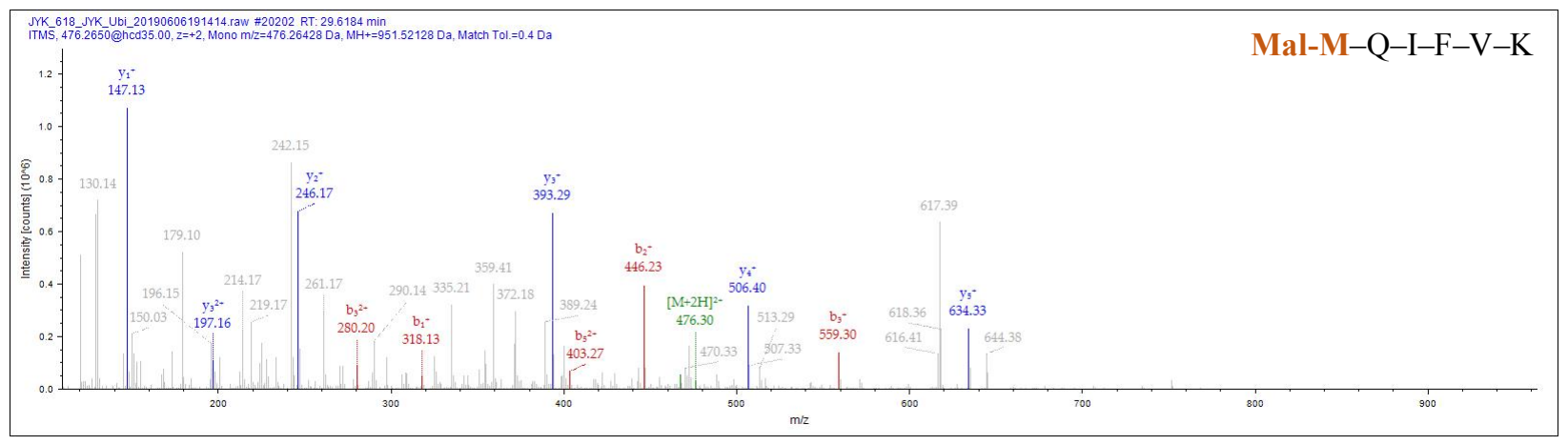




\subsection{2. $\alpha$-lactalbumin}

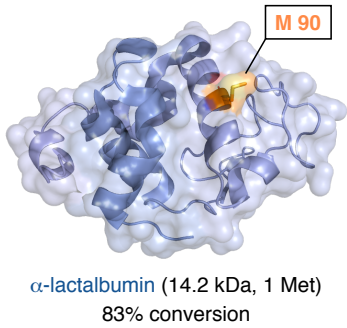

Lactalbumin (21, PDB ID 1F6S) reacted with diethyl ethylidenemalonate (2) under the aforementioned general reaction conditions. The reaction mixture was irradiated for 30 minutes, and then purified with a Zeba spin desalting column (Thermo Scientific, $0.5 \mathrm{~mL}, 7 \mathrm{kDa}$ ) to remove any small molecule reaction components. Conversion (83\%) was determined based on intact protein mass analysis. LC-MS/MS indicated the reaction was selective exclusively for Met 90 . The full protein sequence is shown below, with the relevant peptide highlighted.

$\begin{array}{llllll}{ }^{1} \text { EQLTKCEVFR } & \text { ELKDLKGYGG } & \text { VSLPEWVCTT } & \text { FHTSGYDTQA } & \text { IVQNNDSTEY } & \text { GLFQINNKIW } \\ \text { CKDDQNPHSS } & \text { NICNISCDKF } & \text { LDDDLTDDIM } & \text { CVKKILDKVG } & \text { INYWLAHKAL } & \text { CSEKLDQWLC }\end{array}$

$\mathrm{EKL}^{123}$

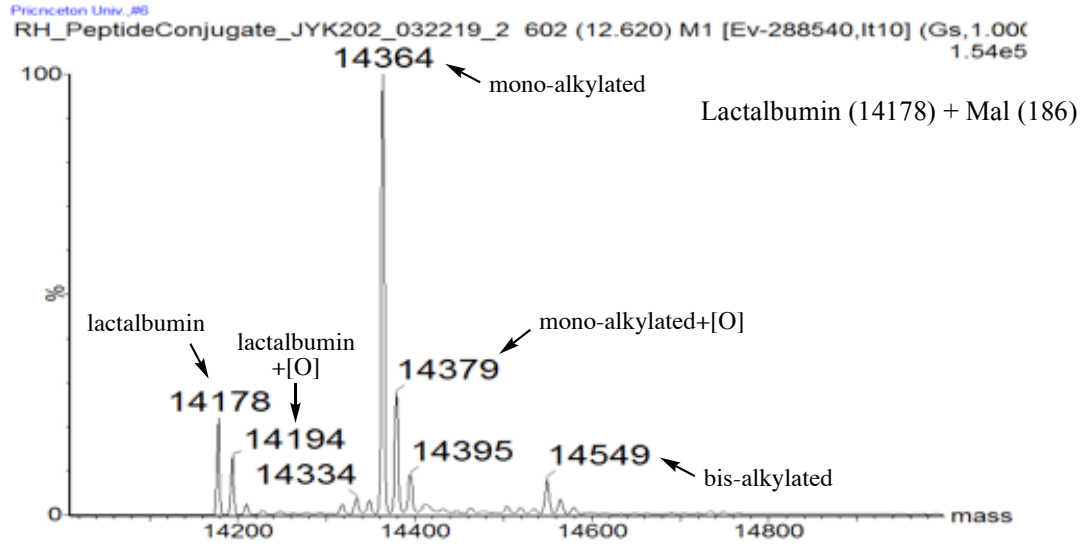




\begin{tabular}{|c|c|}
\hline \multicolumn{1}{|c|}{$\mathbf{b}^{\mathbf{+}}$} & \\
\hline--- & 1 \\
\hline 261.1598 & 2 \\
376.1867 & 3 \\
4991.2136 & 4 \\
606.2406 & 5 \\
719.3246 & 6 \\
820.3723 & 7 \\
935.3993 & 8 \\
1050.4262 & 9 \\
1163.5103 & 10 \\
1480.6395 & 11 \\
1640.6701 & 12 \\
1739.7385 & 13 \\
--- & 14 \\
\hline
\end{tabular}

Sequence

F

L

D

D

D

L

T

D

D

$\underset{\text { Mal-M }}{\mathbf{I}}$

CAM-C

V

K

$\mathbf{y}^{\mathbf{+}}$
---
1738.7756
1625.6916
1510.6646
1395.6377
1280.6107
1167.5267
1066.479
951.4521
836.4251
723.3411
406.2119
246.1812
147.1128

File: RH_PeptideConj_JYK_1_202 03262019 F: FTMS + c ESI d Full ms2 629.2870@hcd27.00 [129.6667-1945.0000] TiC: $4.42 \mathrm{e}+005$ Experimental ( $+3, \mathrm{w}=2, \mathrm{r}=17500$, QExactivePlus)

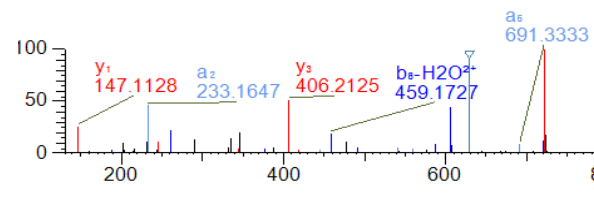

F-L-D-D-D-L-T-D-D-I-Mal-M-CAM-C-V-K

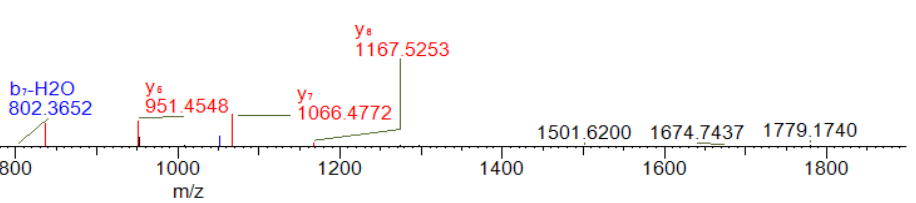




\subsubsection{Myoglobin}

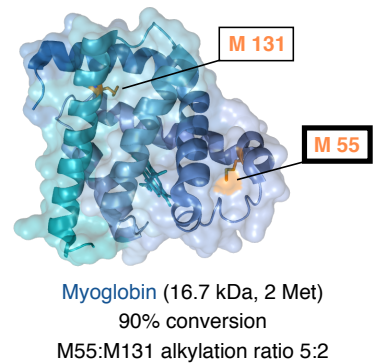

Myoglobin (22, PDB ID 1DWR) reacted with diethyl ethylidenemalonate (2) under the aforementioned general reaction conditions. The reaction mixture was irradiated for 30 minutes, and then purified with a Zeba spin desalting column (Thermo Scientific, $0.5 \mathrm{~mL}, 7 \mathrm{kDa}$ ) to remove any small molecule reaction components. Conversion (90\%) was determined based on intact protein mass analysis. LC-MS/MS indicated the reaction was selective for Met 55 and Met 131. Alkylation ratio was determined by ion count ratio of modified peptides. The full protein sequence is shown below, with the relevant peptide highlighted.

$\begin{array}{llllll}{ }^{1} \text { GLSDGEWQQV } & \text { LNVWGKVEAD } & \text { IAGHGQEVLI } & \text { RLFTGHPETL } & \text { EKFDKFKHLK } & \text { TEAEMKASED } \\ \text { LKKHGTVVLT } & \text { ALGGILKKKG } & \text { HHEAELKPLA } & \text { QSHATKHKIP } & \text { IKYLEFISDA } & \text { IIHVLHSKHP } \\ \text { GDFGADAQGA } & \text { MTKALELFRN } & \text { DIAAKYKELG } & \text { FQG }{ }^{153} & & \end{array}$
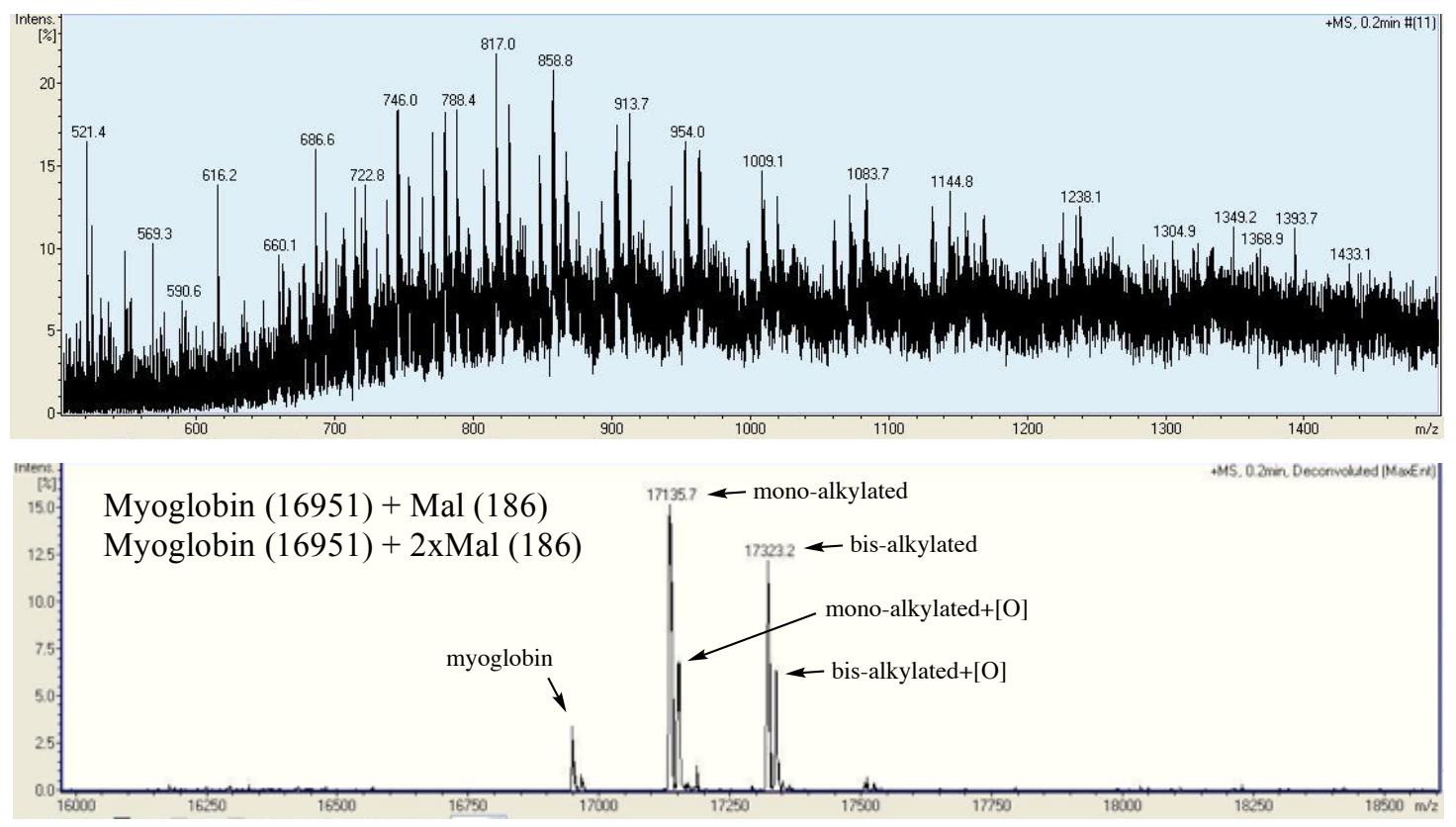


\begin{tabular}{|c|c|c|c|c|c|c|}
\hline $\mathbf{b}^{+}$ & $\mathbf{b}^{2+}$ & & & & $\mathbf{y}^{+}$ & $y^{2+}$ \\
\hline 138.06619 & 69.53673 & 1 & H & 9 & & \\
\hline 251.15025 & 126.07876 & 2 & $\mathbf{L}$ & 8 & 1135.59149 & 568.29939 \\
\hline 379.24522 & 190.12625 & 3 & $\mathbf{K}$ & 7 & 1022.50743 & 511.75735 \\
\hline 480.29289 & 240.65009 & 4 & $\mathbf{T}$ & 6 & 894.41247 & 447.70987 \\
\hline 609.33549 & 305.17138 & 5 & $\mathbf{E}$ & 5 & 793.36479 & 397.18603 \\
\hline 680.37260 & 340.68994 & 6 & A & 4 & 664.32220 & 332.66474 \\
\hline 809.41519 & 405.21124 & 7 & $\mathbf{E}$ & 3 & 593.28508 & 297.14618 \\
\hline \multirow[t]{2}{*}{1126.54488} & 563.77608 & 8 & Mal-M ${ }^{55}$ & 2 & 464.24249 & 232.62488 \\
\hline & & 9 & K & 1 & 147.11280 & 74.06004 \\
\hline
\end{tabular}

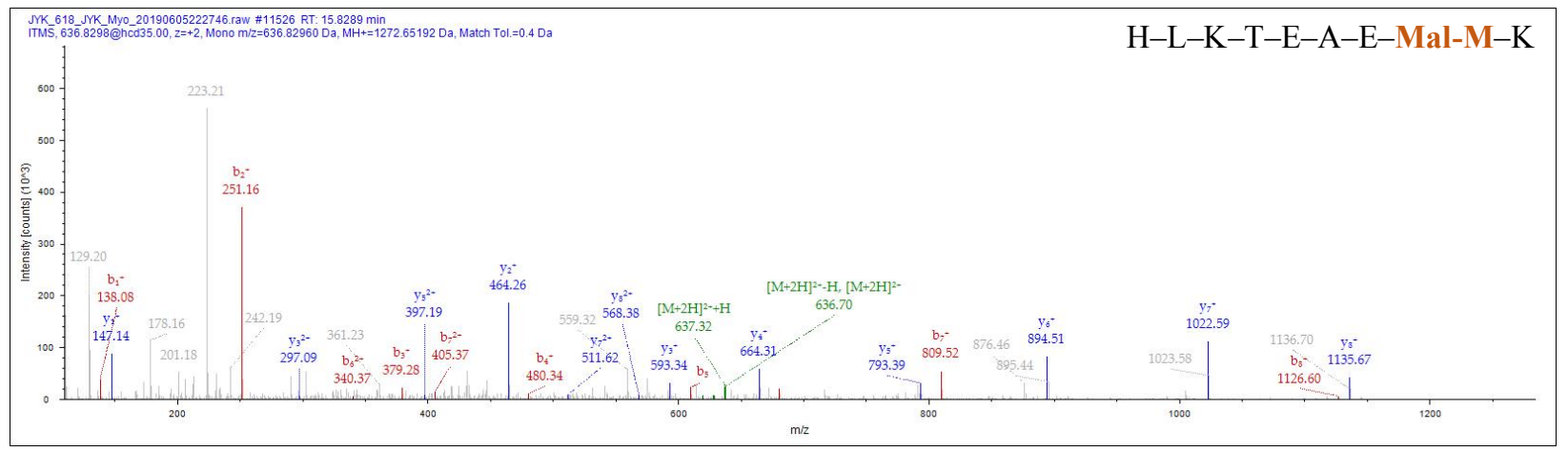




\begin{tabular}{cc|c}
$\mathbf{b}^{+}$ & $\mathbf{b}^{2+}$ & \\
$\mathbf{1 3 8 . 0 6 6 1 9}$ & 69.53673 & 1 \\
$\mathbf{2 3 5 . 1 1 8 9 5}$ & 118.06311 & 2 \\
$\mathbf{2 9 2 . 1 4 0 4 2}$ & 146.57385 & 3 \\
$\mathbf{4 0 7 . 1 6 7 3 6}$ & $\mathbf{2 0 4 . 0 8 7 3 2}$ & 4 \\
$\mathbf{5 5 4 . 2 3 5 7 7}$ & 277.62152 & 5 \\
$\mathbf{6 1 1 . 2 5 7 2 4}$ & 306.13226 & 6 \\
$\mathbf{6 8 2 . 2 9 4 3 5}$ & 341.65081 & 7 \\
$\mathbf{7 9 7 . 3 2 1 2 9}$ & 399.16428 & 8 \\
$\mathbf{8 6 8 . 3 5 8 4 1}$ & 434.68284 & 9 \\
$\mathbf{9 9 6 . 4 1 6 9 8}$ & 498.71213 & 10 \\
$\mathbf{1 0 5 3 . 4 3 8 4 5}$ & $\mathbf{5 2 7 . 2 2 2 8 6}$ & 11 \\
$\mathbf{1 1 2 4 . 4 7 5 5 6}$ & $\mathbf{5 6 2 . 7 4 1 4 2}$ & 12 \\
1441.60525 & 721.30626 & 13 \\
1542.65292 & 771.83010 & 14 \\
& & 15
\end{tabular}

\begin{tabular}{ccccc} 
Sequence & & $\mathbf{y}^{+}$ & $\mathbf{y}^{2+}$ \\
$\mathbf{H}$ & 15 & & \\
$\mathbf{P}$ & 14 & $\mathbf{1 5 5 1 . 6 9 9 5 4}$ & 776.35341 \\
$\mathbf{G}$ & 13 & 1454.64678 & 727.82703 \\
$\mathbf{D}$ & 12 & 1397.62531 & $\mathbf{6 9 9 . 3 1 6 2 9}$ \\
$\mathbf{F}$ & 11 & $\mathbf{1 2 8 2 . 5 9 8 3 7}$ & 641.80282 \\
$\mathbf{G}$ & 10 & $\mathbf{1 1 3 5 . 5 2 9 9 6}$ & 568.26862 \\
$\mathbf{A}$ & 9 & $\mathbf{1 0 7 8 . 5 0 8 4 9}$ & 539.75788 \\
$\mathbf{D}$ & 8 & $\mathbf{1 0 0 7 . 4 7 1 3 8}$ & $\mathbf{5 0 4 . 2 3 9 3 3}$ \\
$\mathbf{A}$ & 7 & $\mathbf{8 9 2 . 4 4 4 4 4}$ & 446.72586 \\
$\mathbf{Q}$ & 6 & $\mathbf{8 2 1 . 4 0 7 3 2}$ & 411.20730 \\
$\mathbf{G}$ & 5 & $\mathbf{6 9 3 . 3 4 8 7 4}$ & 347.17801 \\
$\mathbf{A}$ & 4 & $\mathbf{6 3 6 . 3 2 7 2 8}$ & 318.66728 \\
Mal-M & 3 & $\mathbf{5 6 5 . 2 9 0 1 7}$ & $\mathbf{2 8 3 . 1 4 8 7 2}$ \\
$\mathbf{T}$ & 2 & $\mathbf{2 4 8 . 1 6 0 4 8}$ & 124.58388 \\
$\mathbf{K}$ & 1 & $\mathbf{1 4 7 . 1 1 2 8 0}$ & 74.06004 \\
\hline
\end{tabular}

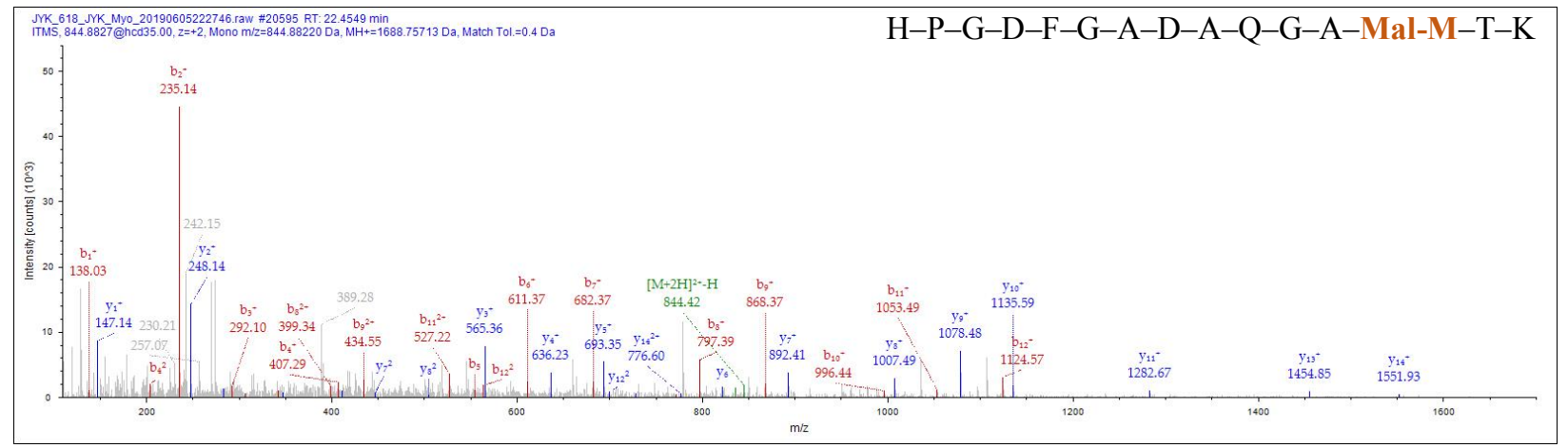




\subsubsection{Human growth hormone (hGH)}

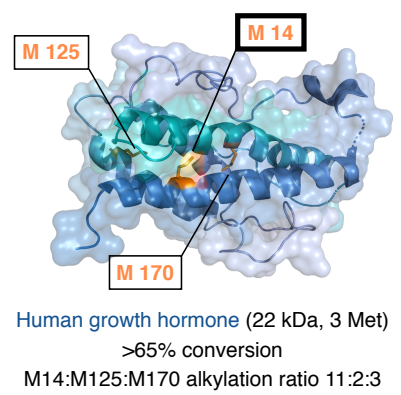

Human growth hormone (23, PDB ID 1HGU) reacted with diethyl ethylidenemalonate (2) under the aforementioned general reaction conditions. $20 \mu \mathrm{M}$ of the protein was used in a reaction instead of $50 \mu \mathrm{M}$. The reaction mixture was irradiated for 30 minutes, and then purified with a Zeba spin desalting column (Thermo Scientific, $0.5 \mathrm{~mL}, 7 \mathrm{kDa})$ to remove any small molecule reaction components. Conversion $(65 \%)$ was determined based on intact protein mass analysis. LC-MS/MS indicated the reaction was selective for Met 14, Met 125, and Met 170. Alkylation ratio was determined by ion count ratio of modified peptides. The full protein sequence is shown below, with the relevant peptide highlighted.

$\begin{array}{llllll}{ }^{1} \text { FPTIPLSRLF } & \text { DNAMLRAHRL } & \text { HQLAFDTYEE } & \text { FEEAYIPKEQ } & \text { KYSFLQAPQA } & \text { SLCFSESIPT } \\ \text { PSNREQAQQK } & \text { SNLQLLRISL } & \text { LLIQSWLEPV } & \text { GFLRSVFANS } & \text { LVYGASDSDV } & \text { YDLLKDLEEG } \\ \text { IQTLMGRLED } & \text { GSPRTGQAFK } & \text { QTYAKFDANS } & \text { HNDDALLKNY } & \text { GLLYCFRKDM } & \text { DKVETFLRIV } \\ \text { QCRSVEGSCG } & \mathrm{F}^{191} & & & \end{array}$
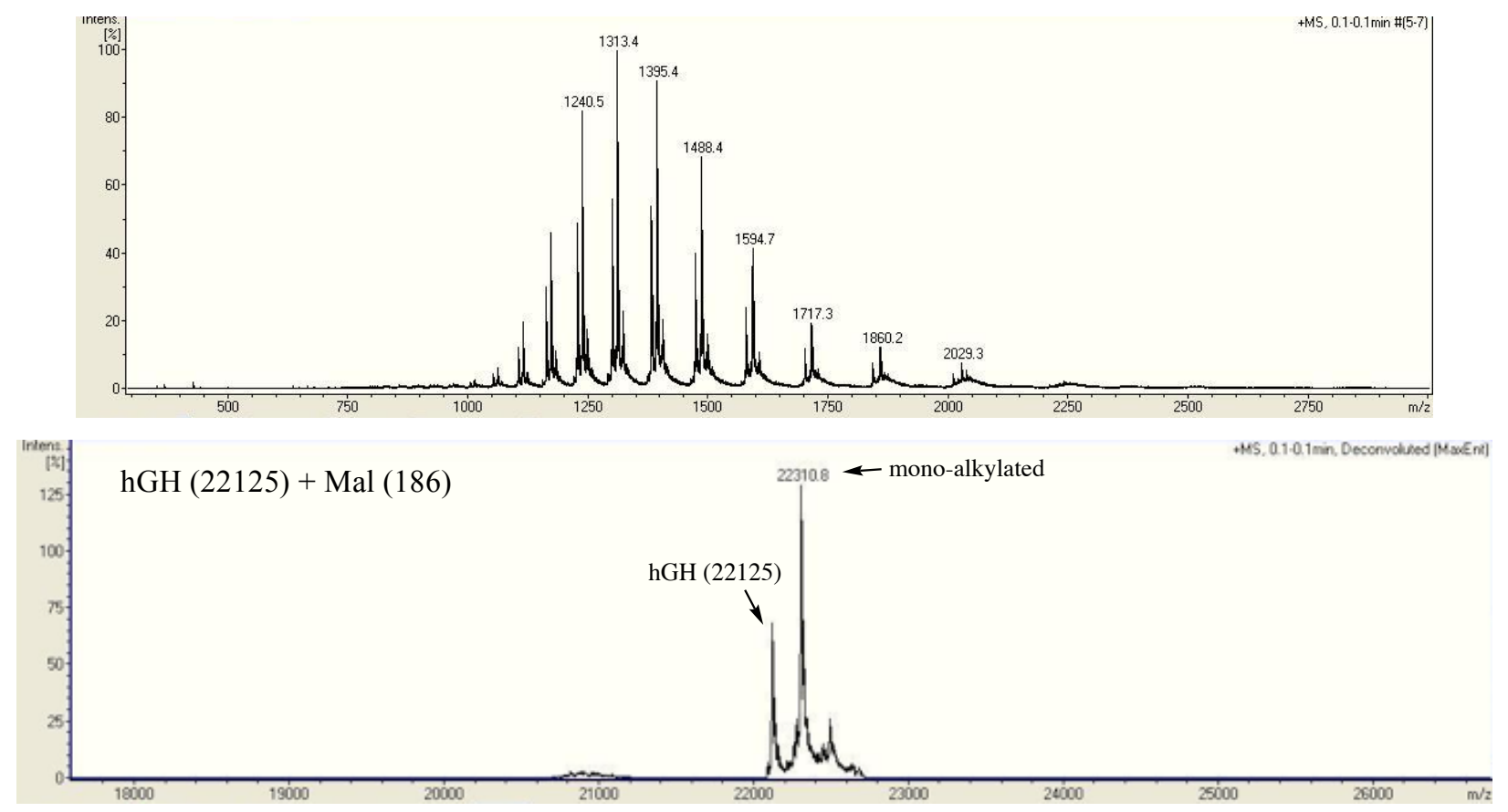


\begin{tabular}{|c|c|c|c|c|c|}
\hline $\mathbf{b}^{+}$ & $\mathbf{b}^{2+}$ & \multicolumn{3}{|c|}{ Sequence } & $\mathbf{y}^{+}$ \\
\hline 114.09134 & 57.54931 & 1 & L & 8 & \\
\hline 261.15975 & 131.08352 & 2 & $\mathbf{F}$ & 7 & 1052.50810 \\
\hline 376.18670 & 188.59699 & 3 & D & 6 & 905.43968 \\
\hline 490.22962 & 245.61845 & 4 & $\mathbf{N}$ & 5 & 790.41274 \\
\hline 561.26674 & 281.13701 & 5 & A & 4 & 676.36981 \\
\hline 878.39642 & 439.70185 & 6 & Mal-M ${ }^{14}$ & 3 & 605.33270 \\
\hline 991.48049 & 496.24388 & 7 & $\mathbf{L}$ & 2 & 288.20302 \\
\hline & & 8 & $\mathbf{R}$ & 1 & 175.11895 \\
\hline
\end{tabular}

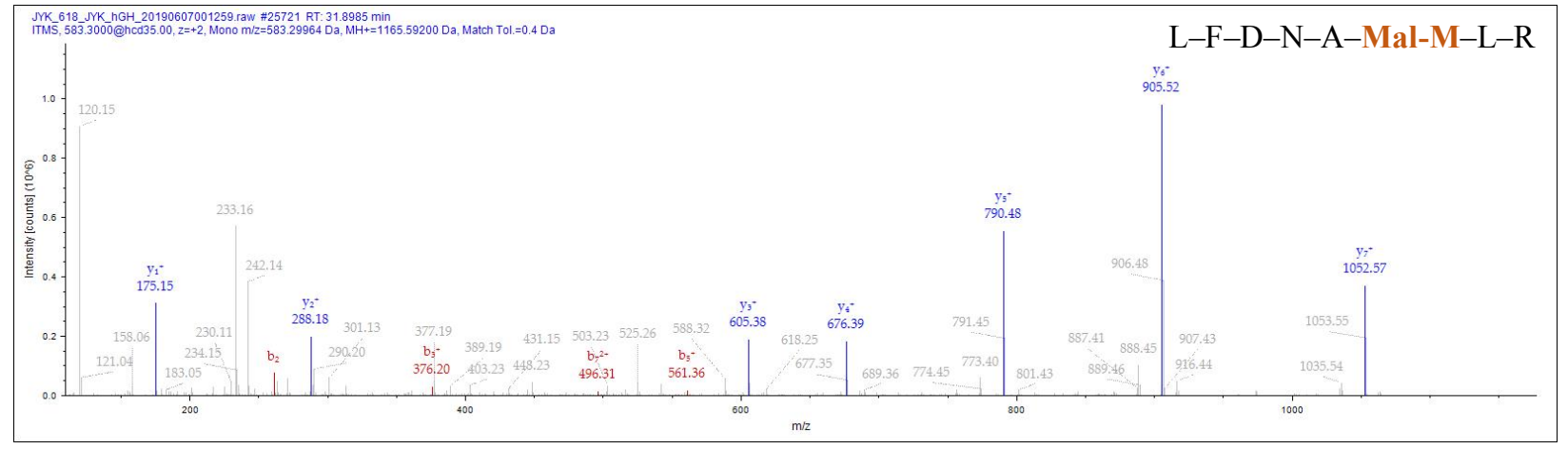




\begin{tabular}{|cc|c}
$\mathbf{b}^{+}$ & $\mathbf{b}^{2+}$ & \\
\hline $\mathbf{1 1 6 . 0 3 4 2 2}$ & 58.52075 & 1 \\
$\mathbf{2 2 9 . 1 1 8 2 8}$ & 115.06278 & 2 \\
$\mathbf{3 5 8 . 1 6 0 8 8}$ & 179.58408 & 3 \\
$\mathbf{4 8 7 . 2 0 3 4 7}$ & 244.10537 & 4 \\
$\mathbf{5 4 4 . 2 2 4 9 3}$ & 272.61610 & 5 \\
$\mathbf{6 5 7 . 3 0 9 0 0}$ & 329.15814 & 6 \\
785.36757 & 393.18743 & 7 \\
886.41525 & 443.71126 & 8 \\
999.49932 & 500.25330 & 9 \\
1316.62900 & 658.81814 & 10 \\
1373.65047 & 687.32887 & 11 \\
& & 12
\end{tabular}

\begin{tabular}{|c|c|c|c|}
\hline Sequence & & $\mathbf{y}^{+}$ & $\mathbf{y}^{2+}$ \\
\hline D & 12 & & \\
\hline $\mathbf{L}$ & 11 & 1432.73520 & 716.87124 \\
\hline $\mathbf{E}$ & 10 & 1319.65113 & 660.32921 \\
\hline $\mathbf{E}$ & 9 & 1190.60854 & 595.80791 \\
\hline G & 8 & 1061.56595 & 531.28661 \\
\hline I & 7 & 1004.54448 & 502.77588 \\
\hline $\mathbf{Q}$ & 6 & 891.46042 & 446.23385 \\
\hline$T$ & 5 & 763.40184 & 382.20456 \\
\hline L & 4 & 662.35416 & 331.68072 \\
\hline Mal-M ${ }^{125}$ & 3 & 549.27010 & 275.13869 \\
\hline G & 2 & 232.14042 & 116.57385 \\
\hline $\mathbf{R}$ & 1 & 175.11895 & 88.06311 \\
\hline
\end{tabular}

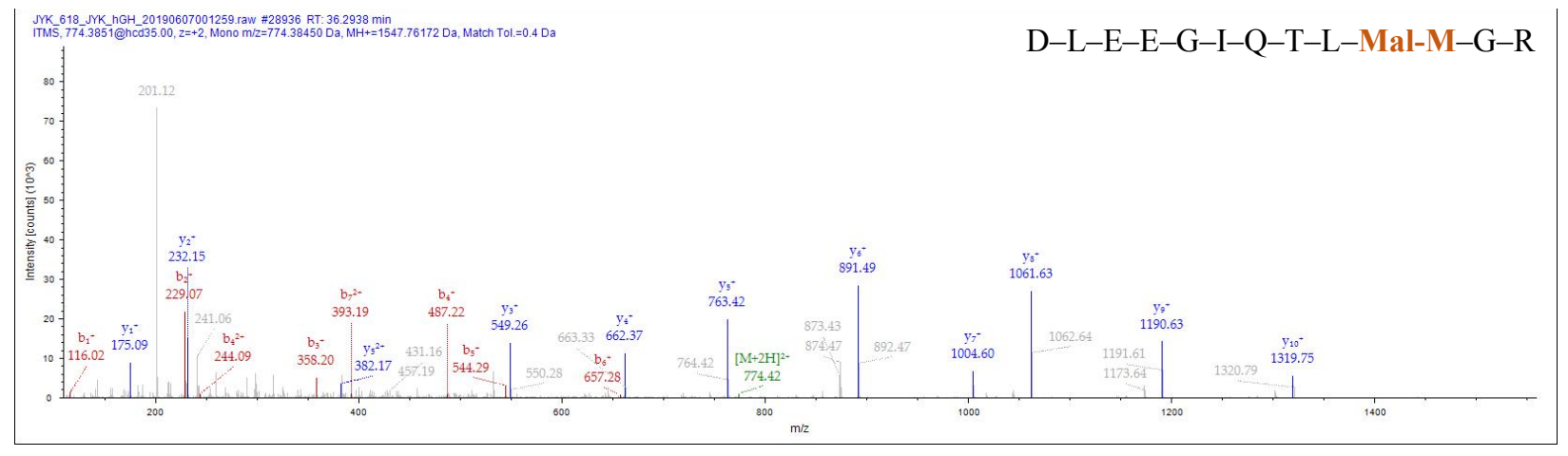




\begin{tabular}{ccccccccc}
$\mathbf{b}^{+}$ & $\mathbf{b}^{2+}$ & $\mathbf{b}^{3+}$ & \multicolumn{3}{c}{ Sequence } & $\mathbf{y}^{+}$ & $\mathbf{y}^{2+}$ & $\mathbf{y}^{3+}$ \\
\hline $\mathbf{1 2 9 . 1 0 2 2 4}$ & 65.05476 & 43.70560 & 1 & $\mathbf{K}$ & 11 & & & \\
$\mathbf{2 4 4 . 1 2 9 1 8}$ & 122.56823 & 82.04791 & 2 & $\mathbf{D}$ & 10 & 1439.70865 & 720.35796 & 480.57440 \\
$\mathbf{5 6 1 . 2 5 8 8 7}$ & 281.13307 & 187.75781 & 3 & Mal-M & 9 & 1324.68171 & $\mathbf{6 6 2 . 8 4 4 4 9}$ & 442.23209 \\
$\mathbf{6 7 6 . 2 8 5 8 1}$ & 338.64654 & 226.10012 & 4 & $\mathbf{D}$ & 8 & $\mathbf{1 0 0 7 . 5 5 2 0 2}$ & $\mathbf{5 0 4 . 2 7 9 6 5}$ & 336.52219 \\
$\mathbf{8 0 4 . 3 8 0 7 7}$ & 402.69402 & 268.79844 & 5 & $\mathbf{K}$ & 7 & $\mathbf{8 9 2 . 5 2 5 0 8}$ & 446.76618 & 298.17988 \\
903.44919 & 452.22823 & 301.82125 & 6 & $\mathbf{V}$ & 6 & 764.43012 & 382.71870 & 255.48156 \\
1032.49178 & 516.74953 & 344.83544 & 7 & $\mathbf{E}$ & 5 & $\mathbf{6 6 5 . 3 6 1 7 0}$ & 333.18449 & $\mathbf{2 2 2 . 4 5 8 7 5}$ \\
1133.53946 & 567.27337 & 378.51800 & 8 & $\mathbf{T}$ & 4 & $\mathbf{5 3 6 . 3 1 9 1 1}$ & 268.66319 & 179.44455 \\
1280.60787 & 640.80757 & 427.54081 & 9 & $\mathbf{F}$ & 3 & 435.27143 & 218.13935 & 145.76199 \\
1393.69194 & 697.34961 & 465.23550 & 10 & $\mathbf{L}$ & 2 & $\mathbf{2 8 8 . 2 0 3 0 2}$ & 144.60515 & 96.73919 \\
& & & 11 & $\mathbf{R}$ & 1 & $\mathbf{1 7 5 . 1 1 8 9 5}$ & 88.06311 & 59.04450
\end{tabular}

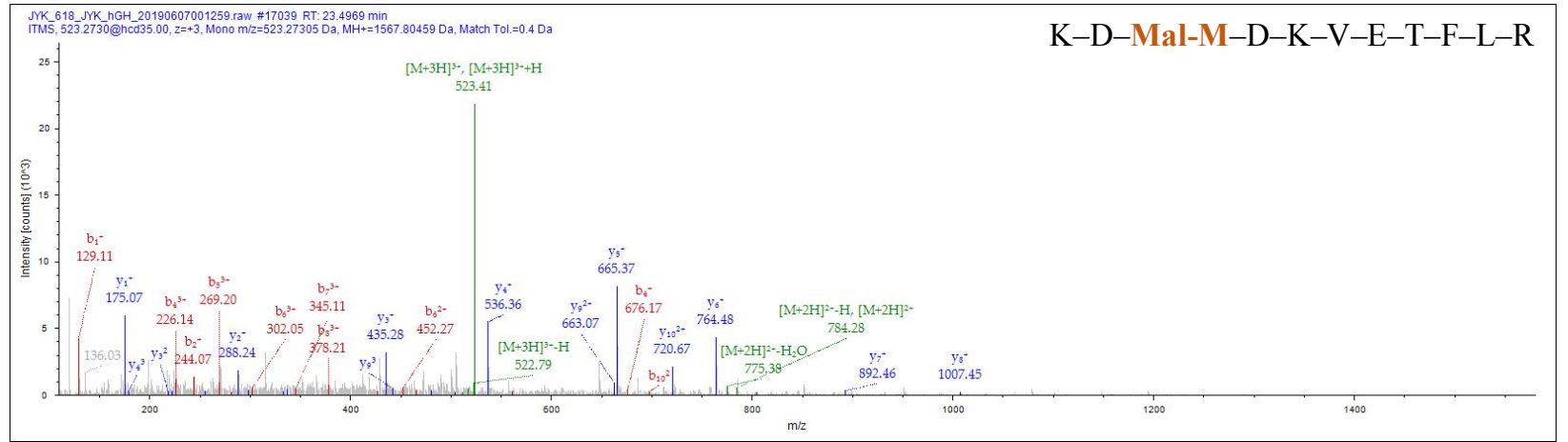




\subsubsection{Carbonic anhydrase (CA)}

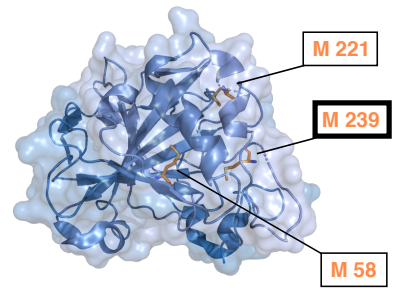

Carbonic anhydrase (29 kDa, 3 Met) $94 \%$ conversion

M58:M221:M239 alkylation ratio 6:1:15

Carbonic anhydrase (24, PDB ID 1V9E) reacted with diethyl ethylidenemalonate (2) under the aforementioned general reaction conditions. The reaction mixture was irradiated for 30 minutes, and then purified with a Zeba spin desalting column (Thermo Scientific, $0.5 \mathrm{~mL}, 7 \mathrm{kDa}$ ) to remove any small molecule reaction components. Conversion (94\%) was determined based on intact protein mass analysis. LC-MS/MS indicated the reaction was selective for Met 58, Met 221, and Met 239. Alkylation ratio was determined by ion count ratio of modified peptides. The full protein sequence is shown below, with the relevant peptide highlighted.

$\begin{array}{llllll}{ }^{1} \text { SHHWGYGKHN } & \text { GPEHWHKDFP } & \text { IANGERQSPV } & \text { DIDTKAVVQD } & \text { PALKPLALVY } & \text { GEATSRRMVN } \\ \text { NGHSFNVEYD } & \text { DSQDKAVLKD } & \text { GPLTGTYRLV } & \text { QFHFHWGSSD } & \text { DQGSEHTVDR } & \text { KKYAAELHLV } \\ \text { HWNTKYGDFG } & \text { TAAQQPDGLA } & \text { VVGVFLKVGD } & \text { ANPALQKVLD } & \text { A LDSIKTKGK } & \text { TDFPNFDPG } \\ \text { S LLPNVLDYW } & \text { TYPGSLTTPP } & \text { LLESVTWIVL } & \text { KEPISVSSQQ } & \text { MLKFRTLNFN } & \text { AEGEPELLML }\end{array}$
ANWRPAQPLK NRQVRGFPK ${ }^{259}$
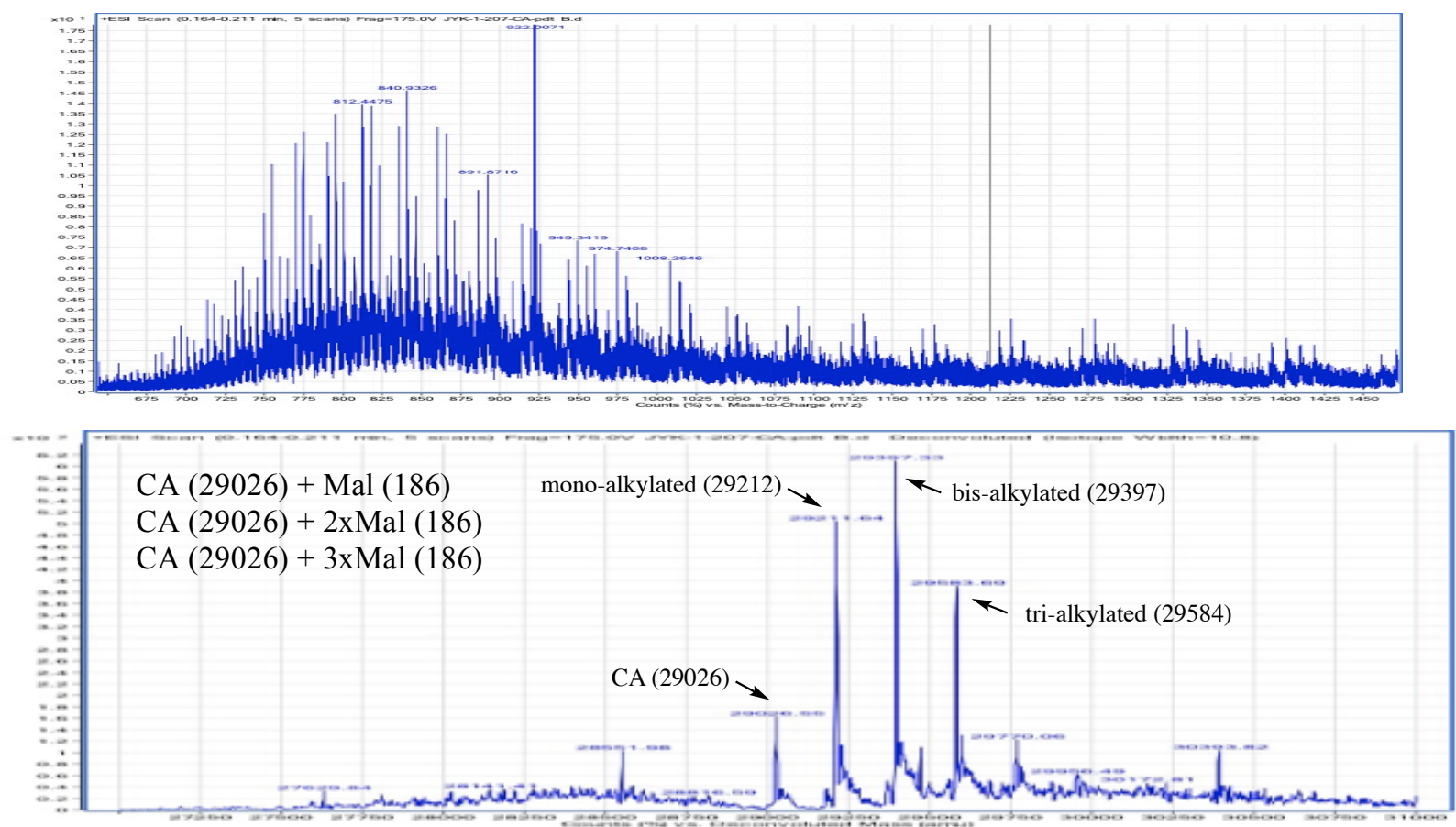


\begin{tabular}{|c|c|c|c|c|c|c|c|c|c|c|}
\hline $\mathbf{b}^{+}$ & $\mathbf{b}^{2+}$ & $\mathbf{b}^{3+}$ & $\mathbf{b}^{4+}$ & & Sequence & & $\mathbf{y}^{+}$ & $\mathbf{y}^{2+}$ & $\mathbf{y}^{3+}$ & $\mathbf{y}^{4+}$ \\
\hline 318.13696 & 159.57212 & 106.71717 & 80.28970 & 1 & Mal-M ${ }^{58}$ & 22 & & & & \\
\hline 417.20538 & 209.10633 & 139.73998 & 105.05680 & 2 & V & 21 & 2379.12114 & 1190.06421 & 793.71190 & 595.53574 \\
\hline 531.24830 & 266.12779 & 177.75429 & 133.56753 & 3 & $\mathbf{N}$ & 20 & 2280.05273 & 1140.53000 & 760.68909 & 570.76864 \\
\hline 645.29123 & 323.14925 & 215.76859 & 162.07826 & 4 & $\mathbf{N}$ & 19 & 2166.00980 & 1083.50854 & 722.67478 & 542.25791 \\
\hline 702.31269 & 351.65999 & 234.77575 & 176.33363 & 5 & G & 18 & 2051.96687 & 1026.48708 & 684.66048 & 513.74718 \\
\hline 839.37161 & 420.18944 & 280.46205 & 210.59836 & 6 & $\mathbf{H}$ & 17 & 1994.94541 & 997.97634 & 665.65332 & 499.49181 \\
\hline 926.40363 & 463.70546 & 309.47273 & 232.35637 & 7 & $\mathbf{S}$ & 16 & 1857.88650 & 929.44689 & 619.96702 & 465.22708 \\
\hline 1073.47205 & 537.23966 & 358.49553 & 269.12347 & 8 & $\mathbf{F}$ & 15 & 1770.85447 & 885.93087 & 590.95634 & 443.46907 \\
\hline 1187.51497 & 594.26113 & 396.50984 & 297.63420 & 9 & $\mathbf{N}$ & 14 & 1623.78606 & 812.39667 & 541.93354 & 406.70197 \\
\hline 1286.58339 & 643.79533 & 429.53265 & 322.40130 & 10 & V & 13 & 1509.74313 & 755.37520 & 503.91923 & 378.19124 \\
\hline 1415.62598 & 708.31663 & 472.54685 & 354.66195 & 11 & $\mathbf{E}$ & 12 & 1410.67471 & 705.84100 & 470.89642 & 353.42414 \\
\hline 1578.68931 & 789.84829 & 526.90129 & 395.42779 & 12 & $\mathbf{Y}$ & 11 & 1281.63212 & 641.31970 & 427.88223 & 321.16349 \\
\hline 1693.71625 & 847.36176 & 565.24360 & 424.18452 & 13 & D & 10 & 1118.56879 & 559.78804 & 373.52778 & 280.39766 \\
\hline 1808.74320 & 904.87524 & 603.58592 & 452.94126 & 14 & D & 9 & 1003.54185 & 502.27456 & 335.18547 & 251.64092 \\
\hline 1895.77522 & 948.39125 & 632.59659 & 474.69926 & 15 & $\mathbf{S}$ & 8 & 888.51491 & 444.76109 & 296.84315 & 222.88418 \\
\hline 2023.83380 & 1012.42054 & 675.28279 & 506.71391 & 16 & $\mathbf{Q}$ & 7 & 801.48288 & 401.24508 & 267.83248 & 201.12618 \\
\hline 2138.86074 & 1069.93401 & 713.62510 & 535.47064 & 17 & D & 6 & 673.42430 & 337.21579 & 225.14628 & 169.11153 \\
\hline 2266.95571 & 1133.98149 & 756.32342 & 567.49438 & 18 & $\mathbf{K}$ & 5 & 558.39736 & 279.70232 & 186.80397 & 140.35480 \\
\hline 2337.99282 & 1169.50005 & 780.00246 & 585.25366 & 19 & A & 4 & 430.30240 & 215.65484 & 144.10565 & 108.33106 \\
\hline 2437.06124 & 1219.03426 & 813.02526 & 610.02077 & 20 & $\mathbf{V}$ & 3 & 359.26528 & 180.13628 & 120.42661 & 90.57178 \\
\hline \multirow[t]{2}{*}{2550.14530} & 1275.57629 & 850.71995 & 638.29178 & 21 & $\mathbf{L}$ & 2 & 260.19687 & 130.60207 & 87.40381 & 65.80467 \\
\hline & & & & 22 & $\mathbf{K}$ & 1 & 147.11280 & 74.06004 & 49.70912 & 37.53366 \\
\hline
\end{tabular}

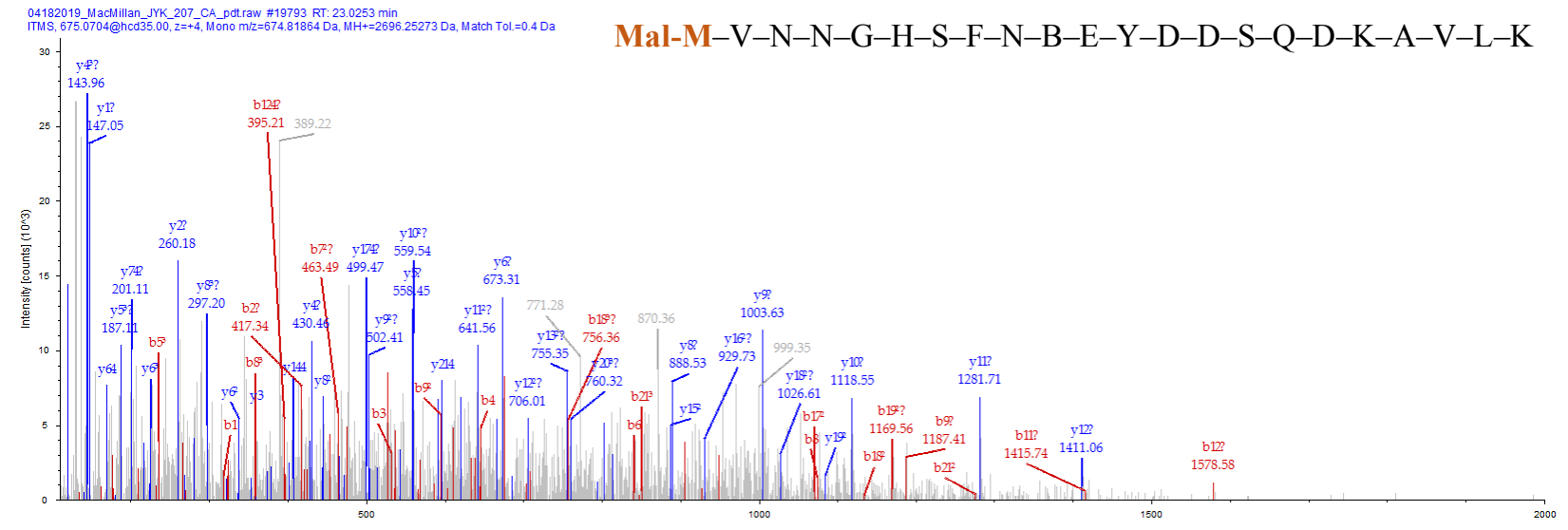




\begin{tabular}{|ccc|}
$\mathbf{b}^{+}$ & $\mathbf{b}^{2+}$ & $\mathbf{b}^{3+}$ \\
\hline $\mathbf{1 3 0 . 0 4 9 8 7}$ & 65.52857 & 44.02147 \\
$\mathbf{2 2 7 . 1 0 2 6 3}$ & 114.05496 & 76.37240 \\
340.18670 & 170.59699 & 114.06708 \\
427.21873 & 214.11300 & 143.07776 \\
$\mathbf{5 2 6 . 2 8 7 1 4}$ & 263.64721 & 176.10056 \\
$\mathbf{6 1 3 . 3 1 9 1 7}$ & 307.16322 & 205.11124 \\
700.35120 & 350.67924 & 234.12192 \\
$\mathbf{8 2 8 . 4 0 9 7 7}$ & 414.70853 & 276.80811 \\
956.46835 & 478.73781 & 319.49430 \\
1273.59804 & 637.30266 & 425.20420 \\
1386.68210 & 693.84469 & 462.89888 \\
1514.77706 & 757.89217 & $\mathbf{5 0 5 . 5 9 7 2 1}$ \\
1661.84548 & 831.42638 & $\mathbf{5 5 4 . 6 2 0 0 1}$ \\
& & \\
\hline
\end{tabular}

Sequence
E
P
I
S
V
S
S
Q
Q
Mal-M
L
K
F
R

$\begin{array}{cccc} & \mathbf{y}^{+} & \mathbf{y}^{2+} & \mathbf{y}^{3+} \\ 14 & & & \\ 13 & 1706.91456 & 853.96092 & 569.64304 \\ 12 & 1609.86180 & 805.43454 & \mathbf{5 3 7 . 2 9 2 1 2} \\ 11 & 1496.77773 & 748.89250 & 499.59743 \\ 10 & 1409.74570 & \mathbf{7 0 5 . 3 7 6 4 9} & 470.58675 \\ 9 & 1310.67729 & \mathbf{6 5 5 . 8 4 2 2 8} & 437.56395 \\ 8 & 1223.64526 & \mathbf{6 1 2 . 3 2 6 2 7} & 408.55327 \\ 7 & 1136.61323 & 568.81025 & 379.54260 \\ 6 & \mathbf{1 0 0 8 . 5 5 4 6 6} & \mathbf{5 0 4 . 7 8 0 9 7} & 336.85640 \\ 5 & \mathbf{8 8 0 . 4 9 6 0 8} & 440.75168 & 294.17021 \\ 4 & \mathbf{5 6 3 . 3 6 6 3 9} & \mathbf{2 8 2 . 1 8 6 8 3} & 188.46032 \\ 3 & 450.28233 & 225.64480 & 150.76563 \\ 2 & 322.18737 & \mathbf{1 6 1 . 5 9 7 3 2} & 108.06731 \\ 1 & \mathbf{1 7 5 . 1 1 8 9 5} & 88.06311 & 59.04450\end{array}$

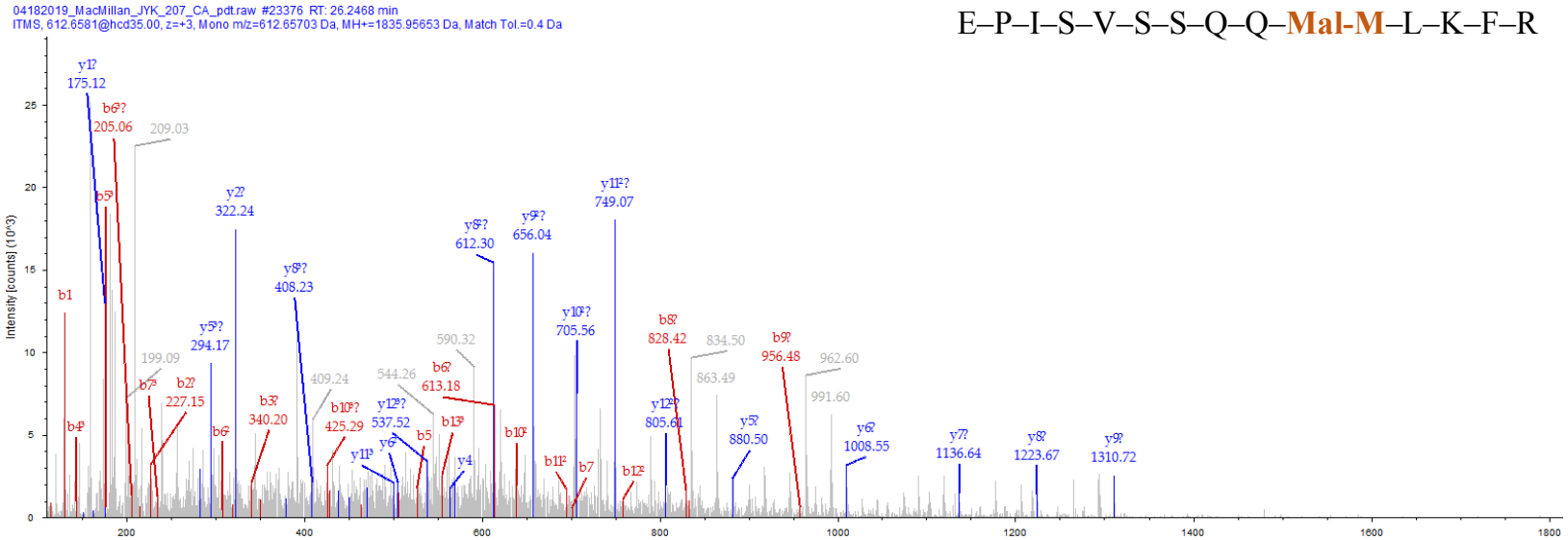




\begin{tabular}{|c|c|c|c|c|c|c|c|c|}
\hline $\mathbf{b}^{+}$ & $\mathbf{b}^{2+}$ & $\mathbf{b}^{3+}$ & & Sequence & & $\mathbf{y}^{+}$ & $\mathbf{y}^{2+}$ & $\mathbf{y}^{3+}$ \\
\hline 102.05496 & 51.53112 & 34.68984 & 1 & $\mathbf{T}$ & 25 & & & \\
\hline 215.13902 & 108.07315 & 72.38452 & 2 & $\mathbf{L}$ & 24 & 2937.51788 & 1469.26258 & 979.84414 \\
\hline 329.18195 & 165.09461 & 110.39883 & 3 & $\mathbf{N}$ & 23 & 2824.43382 & 1412.72055 & 942.14946 \\
\hline 476.25036 & 238.62882 & 159.42164 & 4 & $\mathbf{F}$ & 22 & 2710.39089 & 1355.69908 & 904.13515 \\
\hline 590.29329 & 295.65028 & 197.43595 & 5 & $\mathbf{N}$ & 21 & 2563.32248 & 1282.16488 & 855.11234 \\
\hline 661.33040 & 331.16884 & 221.11498 & 6 & $\mathbf{A}$ & 20 & 2449.27955 & 1225.14341 & 817.09803 \\
\hline 790.37299 & 395.69014 & 264.12918 & 7 & $\mathbf{E}$ & 19 & 2378.24243 & 1189.62486 & 793.41900 \\
\hline 847.39446 & 424.20087 & 283.13634 & 8 & G & 18 & 2249.19984 & 1125.10356 & 750.40480 \\
\hline 976.43705 & 488.72216 & 326.15053 & 9 & $\mathbf{E}$ & 17 & 2192.17838 & 1096.59283 & 731.39764 \\
\hline 1073.48981 & 537.24855 & 358.50146 & 10 & $\mathbf{P}$ & 16 & 2063.13579 & 1032.07153 & 688.38345 \\
\hline 1202.53241 & 601.76984 & 401.51565 & 11 & $\mathbf{E}$ & 15 & 1966.08302 & 983.54515 & 656.03252 \\
\hline 1315.61647 & 658.31187 & 439.21034 & 12 & $\mathbf{L}$ & 14 & 1837.04043 & 919.02385 & 613.01833 \\
\hline 1428.70054 & 714.85391 & 476.90503 & 13 & L & 13 & 1723.95636 & 862.48182 & $\mathbf{5 7 5 . 3 2 3 6 4}$ \\
\hline 1745.83022 & 873.41875 & 582.61492 & 14 & Mal-M $\mathbf{M}^{239}$ & 12 & 1610.87230 & 805.93979 & $\mathbf{5 3 7 . 6 2 8 9 5}$ \\
\hline 1858.91428 & 929.96078 & 620.30961 & 15 & L & 11 & 1293.74262 & 647.37495 & 431.91906 \\
\hline 1929.95140 & 965.47934 & 643.98865 & 16 & $\mathbf{A}$ & 10 & 1180.65855 & 590.83291 & 394.22437 \\
\hline 2043.99433 & 1022.50080 & 682.00296 & 17 & $\mathbf{N}$ & 9 & 1109.62144 & 555.31436 & 370.54533 \\
\hline 2230.07364 & 1115.54046 & 744.02940 & 18 & $\mathbf{W}$ & 8 & 995.57851 & 498.29289 & 332.53102 \\
\hline 2386.17475 & 1193.59101 & 796.06310 & 19 & $\mathbf{R}$ & 7 & 809.49920 & 405.25324 & 270.50458 \\
\hline 2483.22751 & 1242.11739 & 828.41402 & 20 & $\mathbf{P}$ & 6 & 653.39809 & 327.20268 & 218.47088 \\
\hline 2554.26463 & 1277.63595 & 852.09306 & 21 & $\mathbf{A}$ & 5 & 556.34532 & 278.67630 & 186.11996 \\
\hline 2682.32320 & 1341.66524 & 894.77925 & 22 & $\mathbf{Q}$ & 4 & 485.30821 & 243.15774 & 162.44092 \\
\hline 2779.37597 & 1390.19162 & 927.13017 & 23 & $\mathbf{P}$ & 3 & 357.24963 & 179.12845 & 119.75473 \\
\hline \multirow[t]{2}{*}{2892.46003} & 1446.73365 & 964.82486 & 24 & $\mathbf{L}$ & 2 & 260.19687 & 130.60207 & 87.40381 \\
\hline & & & 25 & $\mathbf{K}$ & 1 & 147.11280 & 74.06004 & 49.70912 \\
\hline
\end{tabular}

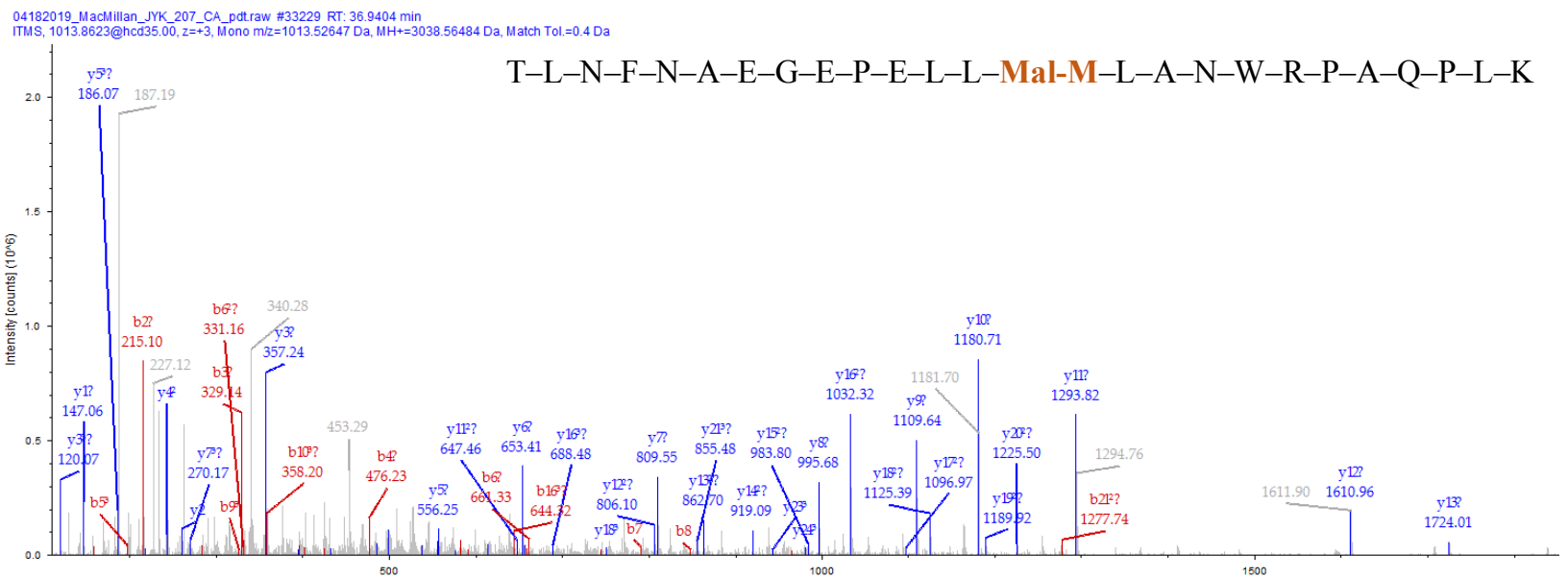




\subsubsection{Ribonuclease A}

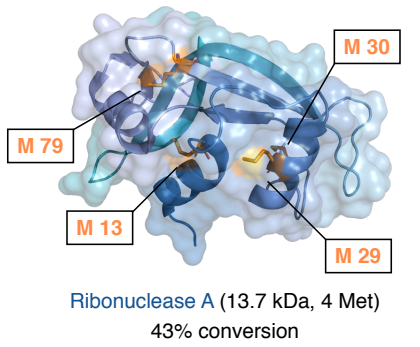

Ribonuclease A (25, PDB ID 1FS3) reacted with diethyl ethylidenemalonate (2) under the aforementioned general reaction conditions. The reaction mixture was irradiated for 30 minutes, and then purified with a Zeba spin desalting column (Thermo Scientific, $0.5 \mathrm{~mL}, 7 \mathrm{kDa}$ ) to remove any small molecule reaction components. Conversion (43\%) was determined based on intact protein mass analysis. LC-MS/MS indicated the reaction was selective for Met 29 and Met 79. Since two methionine residues (Met 29 and Met 30) are located adjacently, LCMS/MS analysis of peptides containing the residues was ambiguous. The full protein sequence is shown below, with the relevant peptide highlighted.

$\begin{array}{llllll}{ }^{1} \text { KETAAAKFER } & \text { QHMDSSTSAA } & \text { SSSNYCNQMM } & \text { KSRNLTKDRC } & \text { KPVNTFVHES } & \text { LADVQAVCSQ } \\ \text { KNVACKNGQT } & \text { NCYQSYSTMS } & \text { ITDCRETGSS } & \text { KYPNCAYKTT } & \text { QANKHIIVAC } & \text { EGNPYVPVHF } \\ \text { DASV }^{124} & & & & \end{array}$
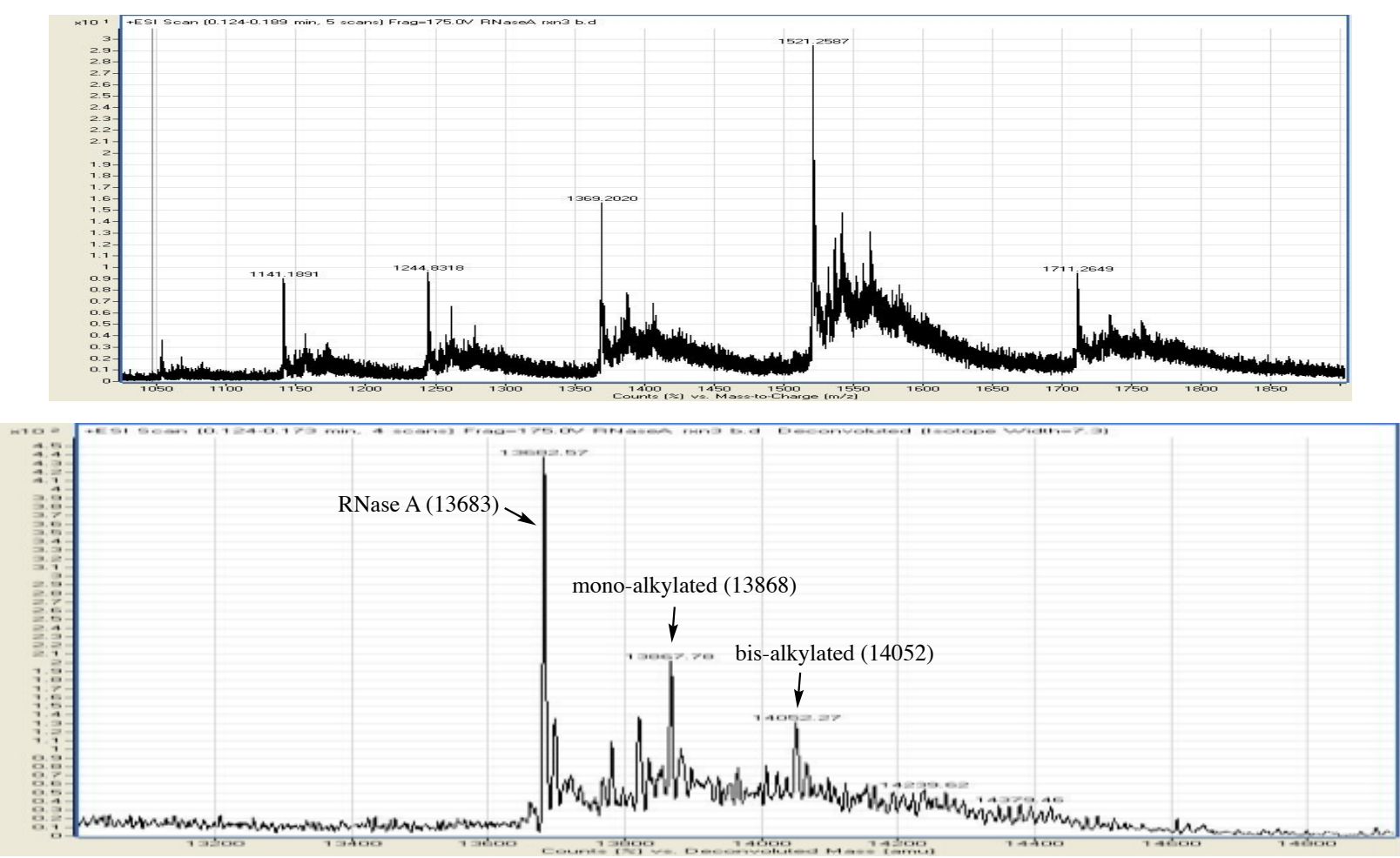


$\begin{array}{cc}\mathbf{b}^{+} & \mathbf{b}^{\mathbf{}+} \\ 112.03931 & 56.52329 \\ 249.09822 & 125.05275 \\ 380.13870 & 190.57299 \\ 495.16564 & 248.08646 \\ 582.19767 & 291.60247 \\ 669.22970 & 335.11849 \\ 770.27738 & 385.64233 \\ 857.30941 & 429.15834 \\ 928.34652 & 464.67690 \\ 999.38364 & 500.19546 \\ 1086.41566 & 543.71147 \\ 1173.44769 & 587.22748 \\ 1260.47972 & 630.74350 \\ 1375.50666 & 688.25697 \\ 1538.56999 & 769.78863 \\ 1698.60064 & 849.80396 \\ 1812.64357 & 906.82542 \\ 1940.70215 & 970.85471 \\ 2257.83183 & 1129.41955 \\ 2388.87231 & 1194.93980 \\ & \end{array}$

1
2
3
4
5
6
7
8
9
10
11
12
13
14
15
16
17
18
19
20
21

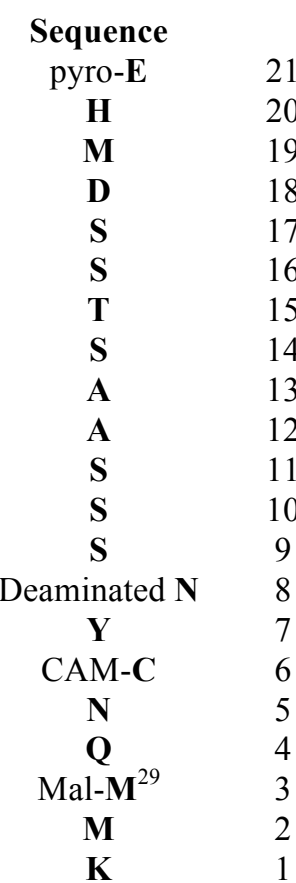

$\mathbf{y}^{+}$
2423.94581
2286.88690
2155.84642
2040.81947
1953.78745
1866.75542
1765.70774
1678.67571
1607.63860
1536.60148
1449.56946
1362.53743
1275.50540
1160.47846
997.41513
837.38448
723.34155
$\mathbf{5 9 5 . 2 8 2 9 7}$
278.15329
$\mathbf{1 4 7 . 1 1 2 8 0}$

$\mathbf{y}^{2+}$

1212.47655

1143.94709

1078.42685

1020.91338

977.39736

933.88135

883.35751

839.84149

804.32294

768.80438

725.28837

681.77235

638.25634

580.74287

499.21120

419.19588

362.17441

298.14513

139.58028

74.06004

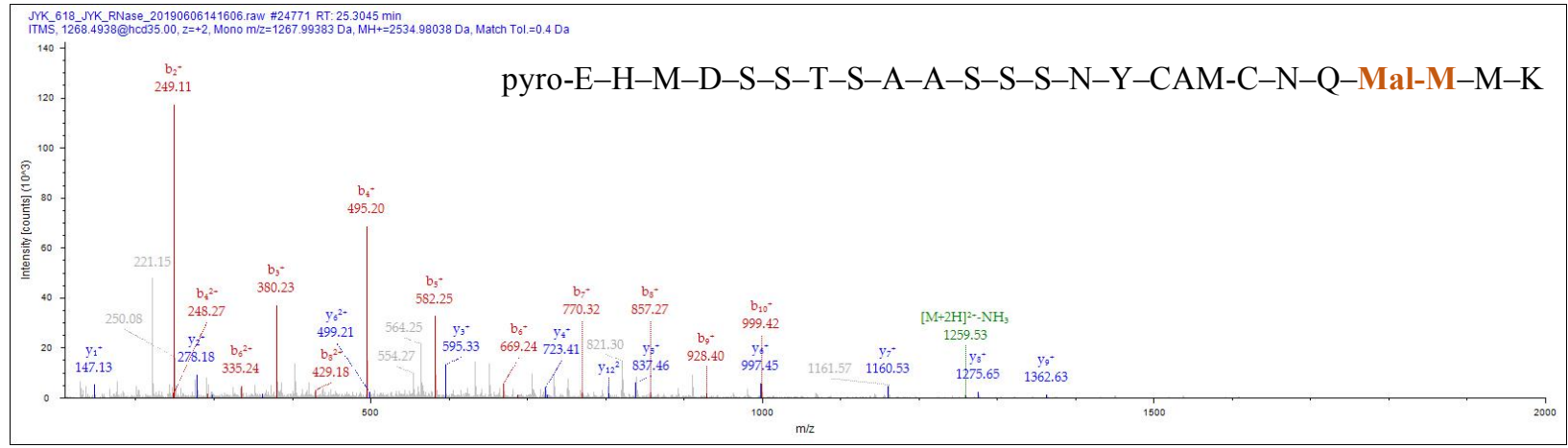




\begin{tabular}{|c|c|c|c|c|c|c|}
\hline $\mathbf{b}^{+}$ & $\mathbf{b}^{2+}$ & \multicolumn{3}{|c|}{ Sequence } & $\mathbf{y}^{+}$ & $\mathbf{y}^{2+}$ \\
\hline 115.05020 & 58.02874 & 1 & $\mathbf{N}$ & 19 & & \\
\hline 172.07167 & 86.53947 & 2 & G & 18 & 2358.95228 & 1179.97978 \\
\hline 301.11426 & 151.06077 & 3 & Deamidated $\mathbf{Q}$ & 17 & 2301.93082 & 1151.46905 \\
\hline 402.16194 & 201.58461 & 4 & $\mathbf{T}$ & 16 & 2172.88822 & 1086.94775 \\
\hline 516.20487 & 258.60607 & 5 & $\mathbf{N}$ & 15 & 2071.84054 & 1036.42391 \\
\hline 676.23552 & 338.62140 & 6 & CAM-C & 14 & 1957.79762 & 979.40245 \\
\hline 839.29884 & 420.15306 & 7 & $\mathbf{Y}$ & 13 & 1797.76697 & 899.38712 \\
\hline 967.35742 & 484.18235 & 8 & $\mathbf{Q}$ & 12 & 1634.70364 & 817.85546 \\
\hline 1054.38945 & 527.69836 & 9 & $\mathbf{S}$ & 11 & 1506.64506 & 753.82617 \\
\hline 1217.45278 & 609.23003 & 10 & $\mathbf{Y}$ & 10 & 1419.61303 & 710.31016 \\
\hline 1304.48481 & 652.74604 & 11 & $\mathbf{S}$ & 9 & 1256.54971 & 628.77849 \\
\hline 1405.53248 & 703.26988 & 12 & $\mathbf{T}$ & 8 & 1169.51768 & 585.26248 \\
\hline 1722.66217 & 861.83472 & 13 & Mal-M ${ }^{79}$ & 7 & 1068.47000 & 534.73864 \\
\hline 1809.69420 & 905.35074 & 14 & $\mathbf{S}$ & 6 & 751.34031 & 376.17380 \\
\hline 1922.77826 & 961.89277 & 15 & I & 5 & 664.30829 & 332.65778 \\
\hline 2023.82594 & 1012.41661 & 16 & $\mathbf{T}$ & 4 & 551.22422 & 276.11575 \\
\hline 2138.85288 & 1069.93008 & 17 & D & 3 & 450.17654 & 225.59191 \\
\hline 2298.88353 & 1149.94540 & 18 & CAM-C & 2 & 335.14960 & 168.07844 \\
\hline & & 19 & $\mathbf{R}$ & 1 & 175.11895 & 88.06311 \\
\hline
\end{tabular}

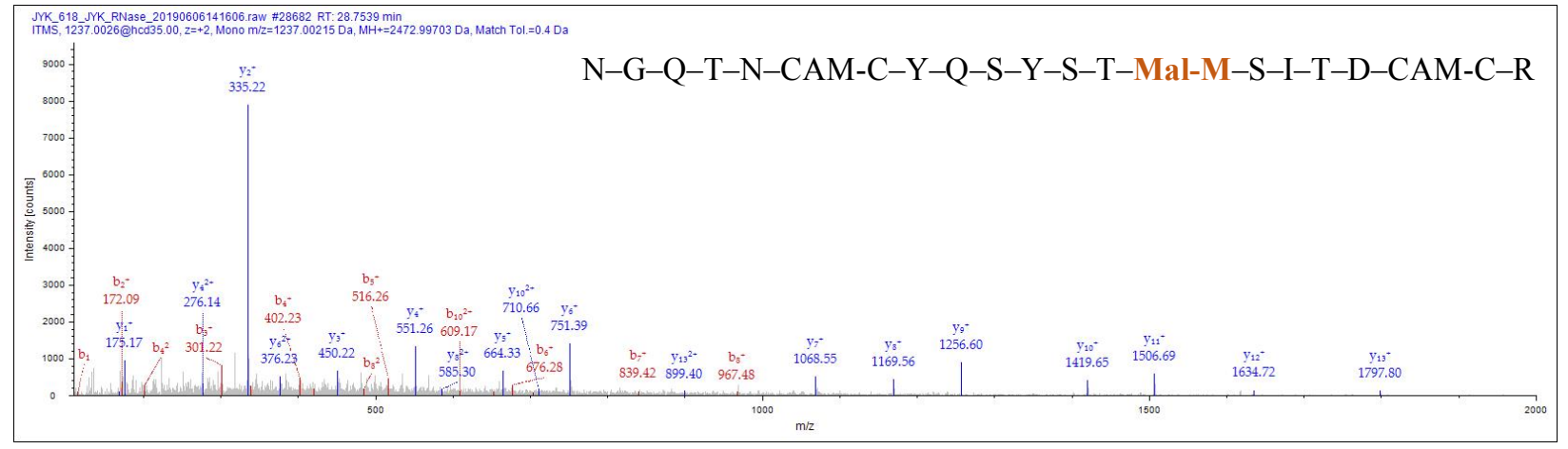




\subsubsection{Enhanced green fluorescent protein (EGFP)}

EGFP reacted with diethyl ethylidenemalonate (2) under the aforementioned general reaction conditions.

The reaction mixture was irradiated for 30 minutes, and then purified with a Zeba spin desalting column (Thermo Scientific, $0.5 \mathrm{~mL}, 7 \mathrm{kDa}$ ) to remove any small molecule reaction components. Conversion ( $>95 \%)$ was determined based on intact protein mass analysis. LC-MS/MS indicated the reaction was selective for Met 79, Met 89, Met 154, Met 219, and Met 234. The full protein sequence is shown below, with the relevant peptide highlighted.

Note: Control experiment without photocatalyst afforded recovery of parent EGFP without observable alkylation of the protein. Furthermore, LC-MS/MS analysis did not identify alkylation on cysteine residues. These results exclude non-photocatalytic alkylation pathway between 2 and nucleophilic residues, such as cysteine and lysine.

$\begin{array}{llllll}{ }^{1} \text { MVSKGEELFT } & \text { GVVPILVELD } & \text { GDVNGHKFSV } & \text { SGEGEGDATY } & \text { GKLTLKFICT } & \text { TGKLPVPWPT } \\ \text { LVTTLTYGVQ } & \text { CFSRYPDHMK } & \text { QHDFFKSAMP } & \text { EGYVQERTIF } & \text { FKDDGNYKTR } & \text { AEVKFEGDTL } \\ \text { VNRIELKGID } & \text { FKEDGNILGH } & \text { KLEYNYNSHN } & \text { VYIMADKQKN } & \text { GIKVNFKIRH } & \text { NIEDGSVQLA } \\ \text { DHYQQNTPIG } & \text { DGPVLLPDNH } & \text { YLSTQSALSK } & \text { DPNEKRDHMV } & \text { LLEFVTAAGI } & \text { TLGMDELYKL }\end{array}$

EHHHHHH ${ }^{247}$
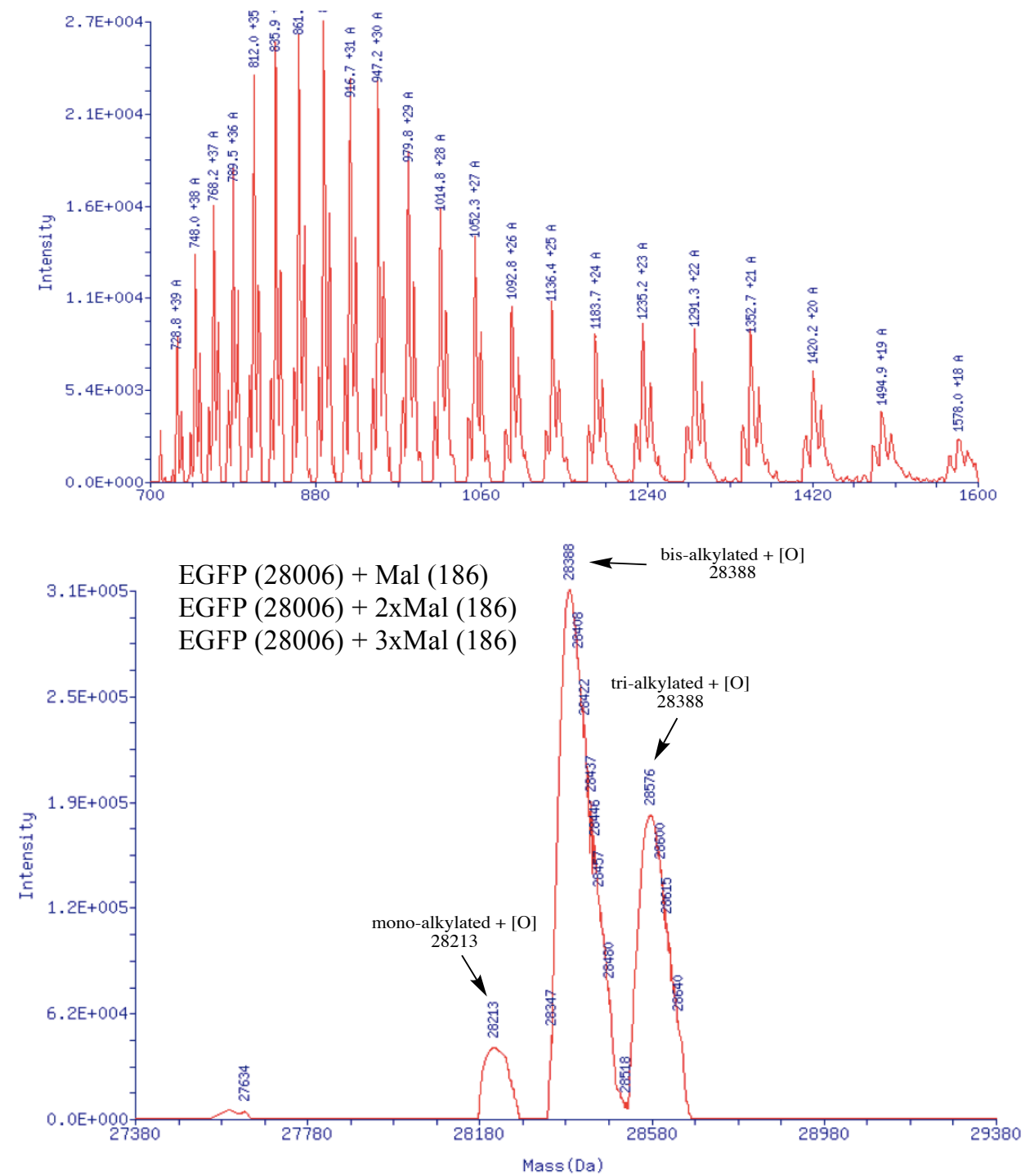


\begin{tabular}{|c|c|c|c|c|c|c|c|c|c|c|}
\hline $\mathbf{b}^{+}$ & $\mathbf{b}^{2+}$ & $\mathbf{b}^{3+}$ & $b^{4+}$ & \multicolumn{3}{|c|}{ Sequence } & $\mathbf{y}^{+}$ & $\mathbf{y}^{2+}$ & $\mathbf{y}^{3+}$ & $y^{4+}$ \\
\hline 164.07061 & 82.53894 & 55.36172 & 41.77311 & 1 & $\mathbf{Y}$ & 12 & & & & \\
\hline 261.12337 & 131.06532 & 87.71264 & 66.03630 & 2 & $\mathbf{P}$ & 11 & 1615.75733 & 808.38230 & 539.25729 & 404.69479 \\
\hline 376.15031 & 188.57879 & 126.05496 & 94.79304 & 3 & D & 10 & 1518.70457 & 759.85592 & 506.90637 & 380.43160 \\
\hline 513.20922 & 257.10825 & 171.74126 & 129.05776 & 4 & $\mathbf{H}$ & 9 & 1403.67762 & 702.34245 & 468.56406 & 351.67486 \\
\hline 830.33891 & 415.67309 & 277.45115 & 208.34018 & 5 & Mal-M ${ }^{79}$ & 8 & 1266.61871 & 633.81299 & 422.87775 & 317.41014 \\
\hline 958.43387 & 479.72057 & 320.14947 & 240.36393 & 6 & K & 7 & 949.48903 & 475.24815 & 317.16786 & 238.12771 \\
\hline 1086.49245 & 543.74986 & 362.83567 & 272.37857 & 7 & $\mathbf{Q}$ & 6 & 821.39406 & 411.20067 & 274.46954 & 206.10397 \\
\hline 1223.55136 & 612.27932 & 408.52197 & 306.64330 & 8 & H & 5 & 693.33549 & 347.17138 & 231.78335 & 174.08933 \\
\hline 1338.57830 & 669.79279 & 446.86429 & 335.40003 & 9 & D & 4 & 556.27657 & 278.64193 & 186.09704 & 139.82460 \\
\hline 1485.64672 & 743.32700 & 495.88709 & 372.16714 & 10 & $\mathbf{F}$ & 3 & 441.24963 & 221.12845 & 147.75473 & 111.06787 \\
\hline 1632.71513 & 816.86120 & 544.90989 & 408.93424 & 11 & $\mathbf{F}$ & 2 & 294.18122 & 147.59425 & 98.73192 & 74.30076 \\
\hline & & & & 12 & $\mathbf{K}$ & 1 & 147.11280 & 74.06004 & 49.70912 & 37.53366 \\
\hline
\end{tabular}

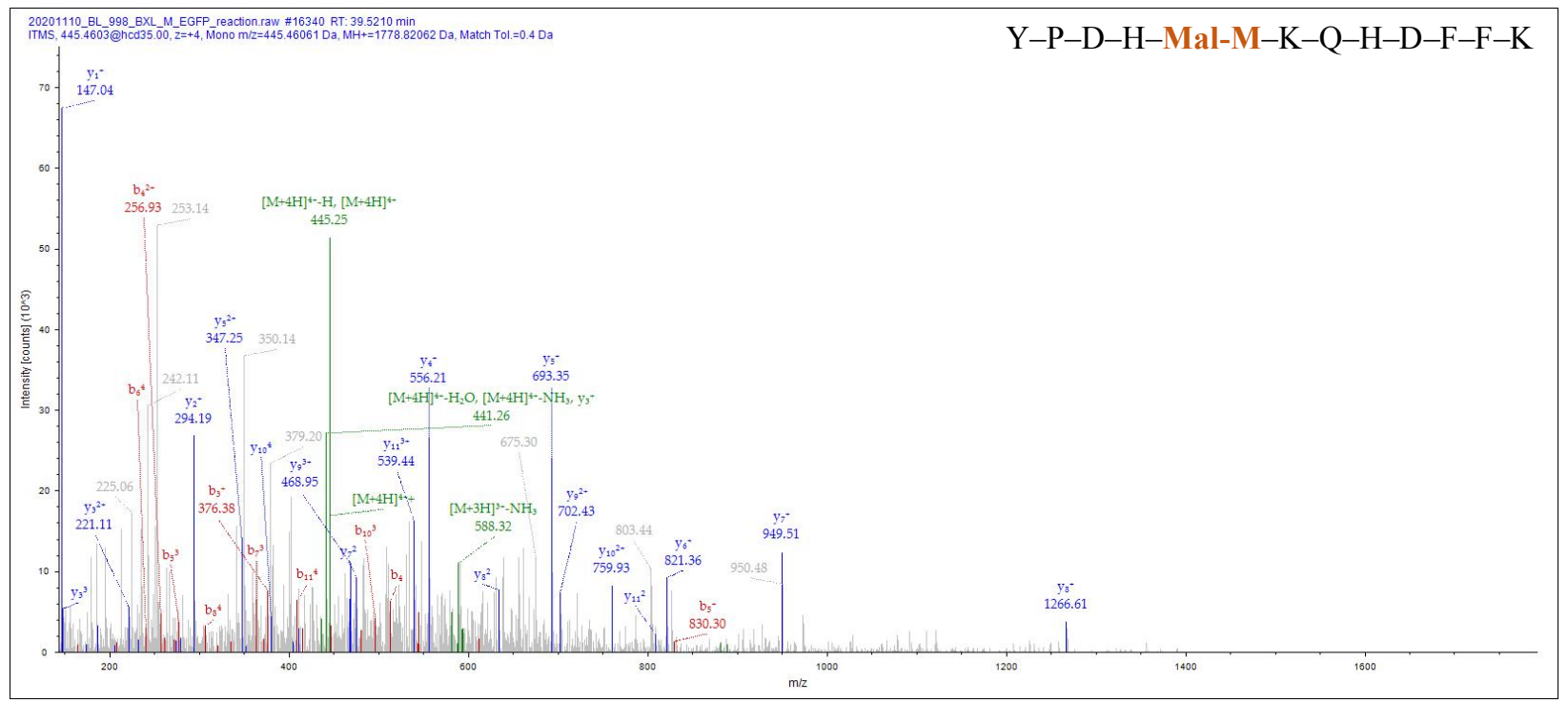




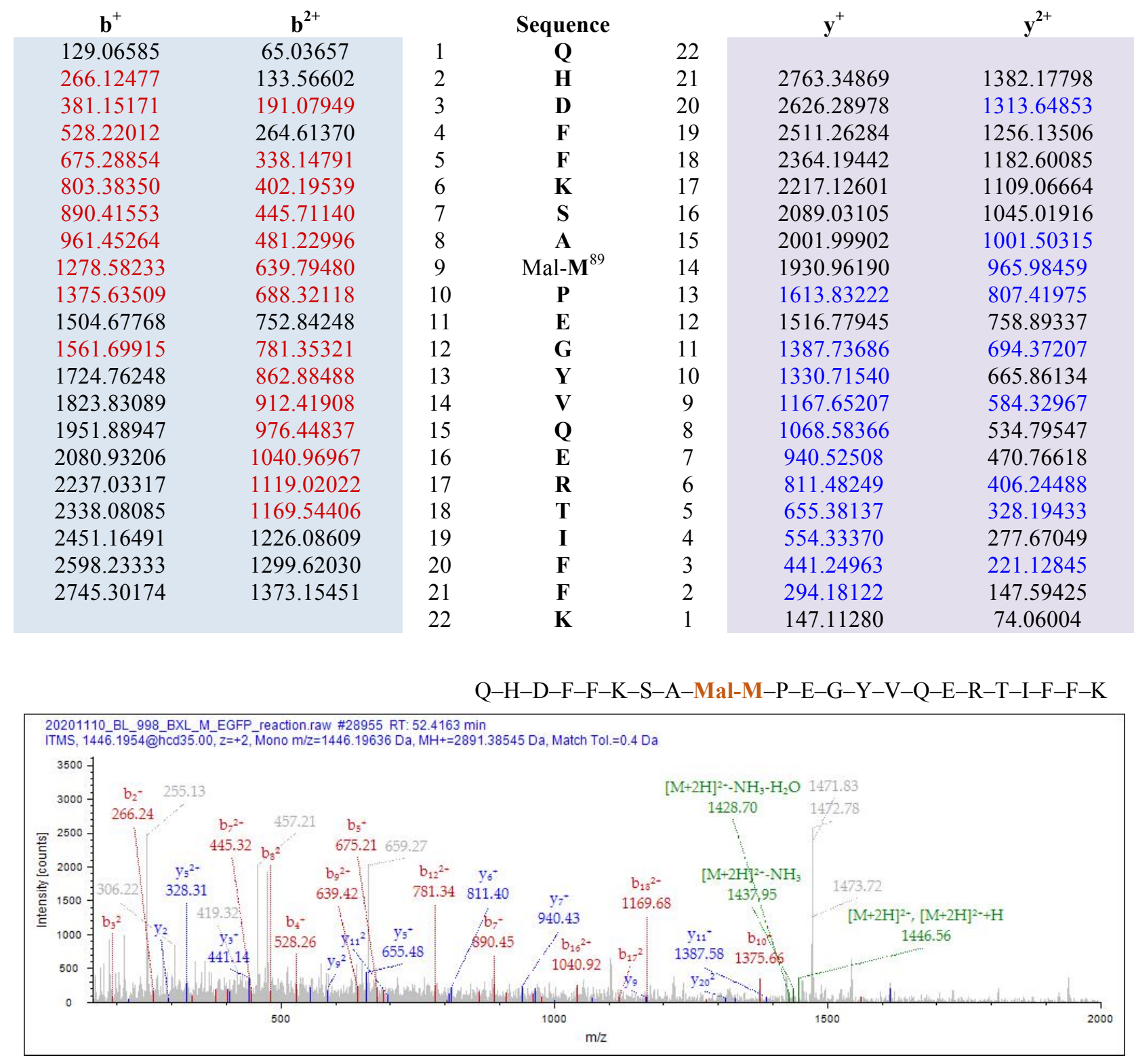




\begin{tabular}{|c|c|c|c|c|c|c|c|c|}
\hline $\mathbf{b}^{+}$ & $\mathbf{b}^{2+}$ & $\mathbf{b}^{3+}$ & \multicolumn{3}{|c|}{ Sequence } & $\mathbf{y}^{+}$ & $\mathbf{y}^{2+}$ & $\mathbf{y}^{3+}$ \\
\hline 114.09134 & 57.54931 & 38.70196 & 1 & L & 16 & & & \\
\hline 243.13393 & 122.07061 & 81.71616 & 2 & $\mathbf{E}$ & 15 & 2233.00053 & 1117.00391 & 745.00503 \\
\hline 406.19726 & 203.60227 & 136.07061 & 3 & $\mathbf{Y}$ & 14 & 2103.95794 & 1052.48261 & 701.99083 \\
\hline 520.24019 & 260.62373 & 174.08491 & 4 & $\mathbf{N}$ & 13 & 1940.89461 & 970.95094 & 647.63639 \\
\hline 683.30352 & 342.15540 & 228.43936 & 5 & $\mathbf{Y}$ & 12 & 1826.85169 & 913.92948 & 609.62208 \\
\hline 797.34645 & 399.17686 & 266.45367 & 6 & $\mathbf{N}$ & 11 & 1663.78836 & 832.39782 & 555.26764 \\
\hline 884.37847 & 442.69288 & 295.46434 & 7 & $\mathbf{S}$ & 10 & 1549.74543 & 775.37635 & 517.25333 \\
\hline 1021.43739 & 511.22233 & 341.15065 & 8 & H & 9 & 1462.71340 & 731.86034 & 488.24265 \\
\hline 1135.48031 & 568.24379 & 379.16496 & 9 & $\mathbf{N}$ & 8 & 1325.65449 & 663.33088 & 442.55635 \\
\hline 1234.54873 & 617.77800 & 412.18776 & 10 & $\mathbf{V}$ & 7 & 1211.61156 & 606.30942 & 404.54204 \\
\hline 1397.61206 & 699.30967 & 466.54220 & 11 & $\mathbf{Y}$ & 6 & 1112.54315 & 556.77521 & 371.51923 \\
\hline 1510.69612 & 755.85170 & 504.23689 & 12 & I & 5 & 949.47982 & 475.24355 & 317.16479 \\
\hline 2013.91501 & 1007.46115 & 671.97652 & 13 & Mal-M ${ }^{154}$ & 4 & 836.39576 & 418.70152 & 279.47010 \\
\hline 2084.95213 & 1042.97970 & 695.65556 & 14 & A & 3 & 333.17686 & 167.09207 & 111.73047 \\
\hline 2199.97907 & 1100.49317 & 733.99787 & 15 & D & 2 & 262.13975 & 131.57351 & 88.05143 \\
\hline & & & 16 & $\mathbf{K}$ & 1 & 147.11280 & 74.06004 & 49.70912 \\
\hline
\end{tabular}

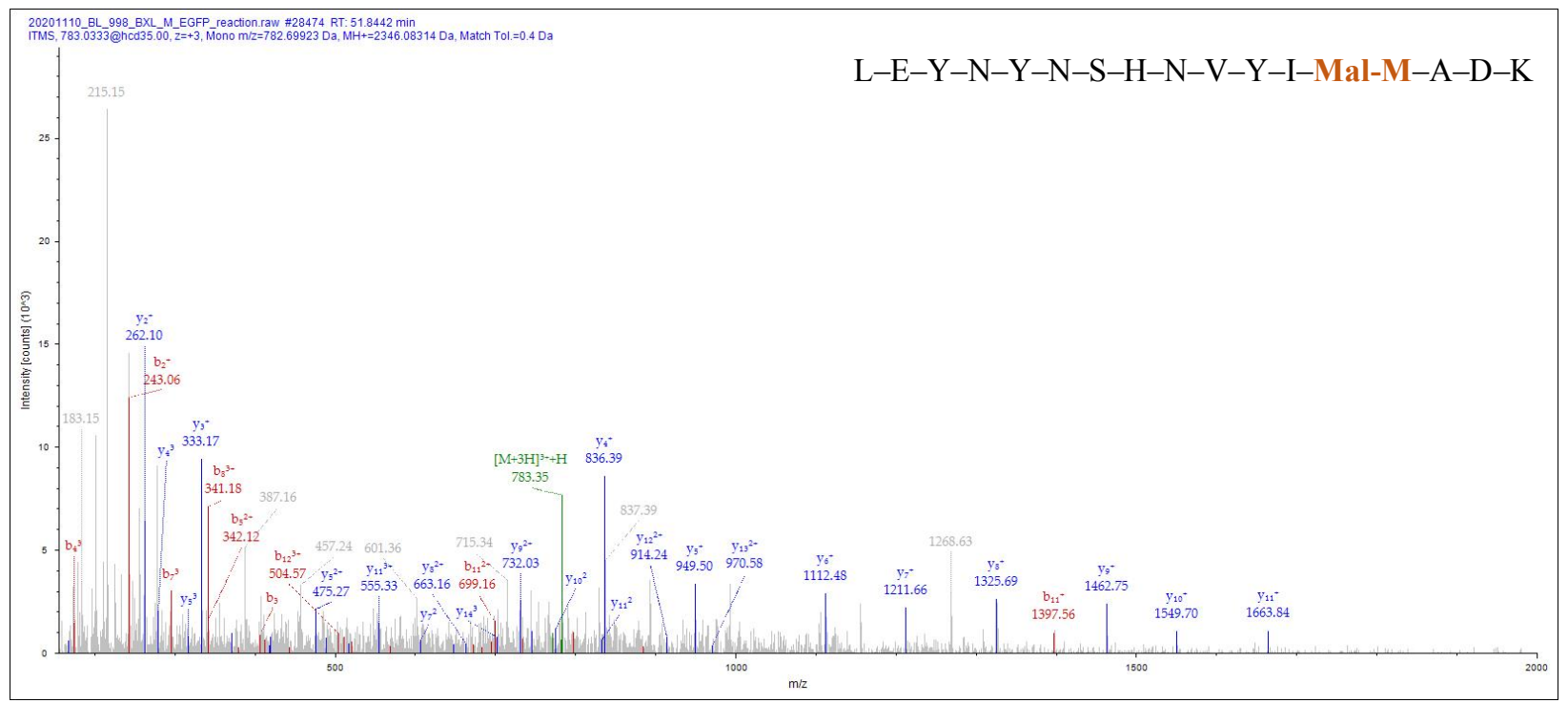




\begin{tabular}{ccc}
$\mathbf{b}^{+}$ & $\mathbf{b}^{2+}$ & $\mathbf{b}^{3+}$ \\
\hline 157.10839 & 79.05783 & 53.04098 \\
272.13533 & 136.57130 & 91.38329 \\
409.19424 & 205.10076 & 137.06960 \\
726.32393 & 363.66560 & 242.77949 \\
825.39234 & 413.19981 & 275.80230 \\
938.47640 & 469.74184 & 313.49699 \\
1051.56047 & 526.28387 & 351.19167 \\
1180.60306 & 590.80517 & 394.20587 \\
1327.67148 & 664.33938 & 443.22868 \\
1426.73989 & 713.87358 & 476.25148 \\
1527.78757 & 764.39742 & 509.93404 \\
1598.82468 & 799.91598 & 533.61308 \\
1669.86180 & 835.43454 & 557.29212 \\
1726.88326 & 863.94527 & 576.29927 \\
1839.96732 & 920.48730 & 613.99396 \\
1941.01500 & 971.01114 & 647.67652 \\
2054.09907 & 1027.55317 & 685.37121 \\
2111.12053 & 1056.06390 & 704.37836 \\
2428.25021 & 1214.62875 & 810.08826 \\
2543.27716 & 1272.14222 & 848.43057 \\
2672.31975 & 1336.66351 & 891.44477 \\
2785.40381 & 1393.20555 & 929.13946 \\
& & \\
\hline
\end{tabular}

\begin{tabular}{|c|c|c|c|c|c|}
\hline & Sequence & & $\mathbf{y}^{+}$ & $\mathbf{y}^{2+}$ & $\mathbf{y}^{3+}$ \\
\hline 1 & $\mathbf{R}$ & 23 & & & \\
\hline 2 & D & 22 & 2810.37660 & 1405.69194 & 937.46372 \\
\hline 3 & H & 21 & 2695.34965 & 1348.17846 & 899.12140 \\
\hline 4 & Mal-M $\mathbf{M}^{219}$ & 20 & 2558.29074 & 1279.64901 & 853.43510 \\
\hline 5 & V & 19 & 2241.16106 & 1121.08417 & 747.72520 \\
\hline 6 & $\mathbf{L}$ & 18 & 2142.09264 & 1071.54996 & 714.70240 \\
\hline 7 & $\mathbf{L}$ & 17 & 2029.00858 & 1015.00793 & 677.00771 \\
\hline 8 & $\mathbf{E}$ & 16 & 1915.92451 & 958.46590 & 639.31302 \\
\hline 9 & F & 15 & 1786.88192 & 893.94460 & 596.29882 \\
\hline 10 & V & 14 & 1639.81351 & 820.41039 & 547.27602 \\
\hline 11 & $\mathbf{T}$ & 13 & 1540.74509 & 770.87619 & 514.25322 \\
\hline 12 & $\mathbf{A}$ & 12 & 1439.69742 & 720.35235 & 480.57066 \\
\hline 13 & $\mathbf{A}$ & 11 & 1368.66030 & 684.83379 & 456.89162 \\
\hline 14 & G & 10 & 1297.62319 & 649.31523 & 433.21258 \\
\hline 15 & I & 9 & 1240.60172 & 620.80450 & 414.20543 \\
\hline 16 & $\mathbf{T}$ & 8 & 1127.51766 & 564.26247 & 376.51074 \\
\hline 17 & $\mathbf{L}$ & 7 & 1026.46998 & 513.73863 & 342.82818 \\
\hline 18 & G & 6 & 913.38592 & 457.19660 & 305.13349 \\
\hline 19 & Mal-M ${ }^{234}$ & 5 & 856.36445 & 428.68587 & 286.12634 \\
\hline 20 & D & 4 & 539.23477 & 270.12102 & 180.41644 \\
\hline 21 & $\mathbf{E}$ & 3 & 424.20783 & 212.60755 & 142.07413 \\
\hline 22 & $\mathbf{L}$ & 2 & 295.16523 & 148.08626 & 99.05993 \\
\hline 23 & $\mathbf{Y}$ & 1 & 182.08117 & 91.54422 & 61.36524 \\
\hline
\end{tabular}

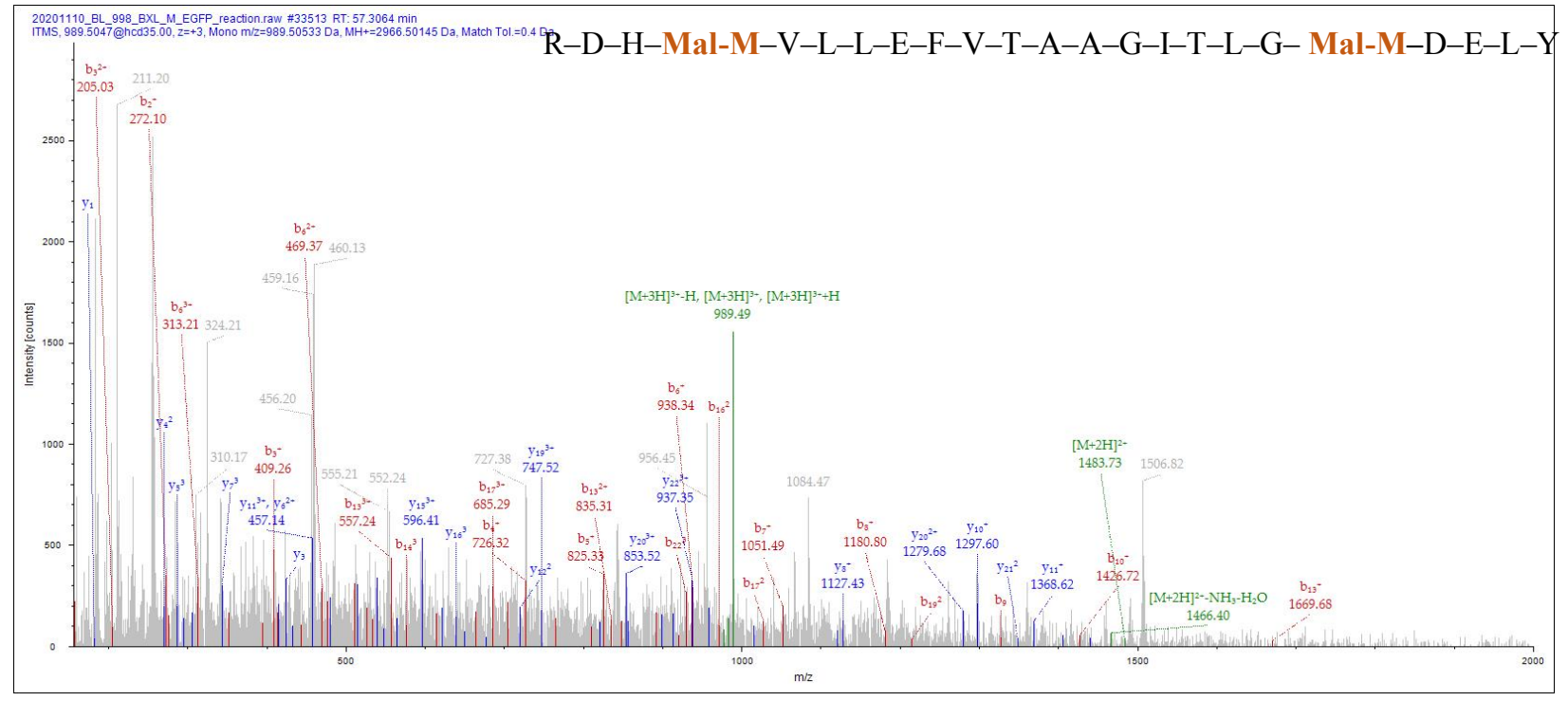




\section{Modification of EGFP}

\subsection{Experimental procedures}

\subsubsection{General reaction conditions for methionine alkylation on EGFR}

Enhanced green fluorescent protein (EGFP) was purchased from Chromotek. Milli-Q water and DMF were degassed by the freeze-pump-thaw method ( 5 cycles). All stock solutions except the EGFP solution were prepared with degassed solvents and stored in a glovebox. To a V-shaped vial insert (VWR, $200 \mu \mathrm{L}$ ) equipped with a stir bar was added EGFP solution (0.2 mM in $\left.\mathrm{H}_{2} \mathrm{O}, 10 \mu \mathrm{L}, 2 \mathrm{nmol}\right)$, PBS stock solution (Gibco, 10x, $\mathrm{pH} 7.4,10 \mu \mathrm{L}$ ), lumiflavin solution (10 mM in $\left.\mathrm{H}_{2} \mathrm{O}, 5 \mu \mathrm{L}, 50 \mathrm{nmol}\right)$, Michael acceptor solution $(17,0.2 \mathrm{M}$ in DMF, $5 \mu \mathrm{L}, 1 \mu \mathrm{mol})$ and water $(70 \mu \mathrm{L})$. To a $2 \mathrm{~mL}$ vial was added $\mathrm{H}_{2} \mathrm{O}$ as a coolant and the vial insert containing reaction mixture. The vial was sealed and brought out of the glovebox. The reaction was stirred and irradiated using a $40 \mathrm{~W}$ Kessil blue LED lamp (PR160-440, 100\% intensity, $4 \mathrm{~cm}$ away) for $30 \mathrm{~min}$. The reaction was filtered through centrifuge tube filters (Corning Costar, Fisher Scientific) and the filtrate was purified by spin desalting columns (Zeba, Thermo Fisher, 0.5 mL. 7K MWCO). Purified product was subjected to LC-MS for intact mass analysis.

\subsubsection{Click reactions on alkyne attached EGFP}

The procedure developed by Finn et al. was used. ${ }^{2}$ To a EGFP-alkyne 26 solution in PBS buffer $(250 \mu \mathrm{L})$ was added an azide stock solution $(\mathbf{2 7}$ or $\mathbf{2 8}, 100 \mu \mathrm{M}, 2.5 \mu \mathrm{L}) .28$ was prepared following a known procedure. ${ }^{3}$ To a separate tube was sequentially mixed aminoguanidine hydrochloride ( $1 \mathrm{mM}$ in water, $2.5 \mu \mathrm{L}), \mathrm{CuSO}_{4}(100 \mu \mathrm{M}$ in water, $2.5 \mu \mathrm{L})$, THPTA $(500 \mu \mathrm{M}$ in water, $2.5 \mu \mathrm{L})$, and freshly made sodium ascorbate $(5 \mathrm{mM}$ in water, $2.5 \mu \mathrm{L})$. Then, the copper solution was transferred to the EGFP-alkyne solution and briefly mixed by vortexing. The reaction was incubated at room temperature for $1 \mathrm{~h}$ and quenched by adding EDTA ( $5 \mathrm{mM}, 25 \mu \mathrm{L})$. The reaction was purified by spin desalting columns (Zeba, Thermo Fisher, $0.5 \mathrm{~mL}$. 7K MWCO). Purified product was analyzed by western blotting.

\subsubsection{Cell culture and imaging}

Cells were grown in the Muir group's culture room at the Princeton University Department of Chemistry. SJSA-1 cells were cultured in DMEM supplemented with FBS, glutamine and Pen-Strep. Cells were plated on 4well chamber slides (Lab-Tek, Thermo Fisher) a day prior to imaging. At a desired confluency, cells were treated with $20 \sim 30 \mu \mathrm{g} / \mathrm{mL}$ of EGFP-(Arg) $)_{9}$ in DMEM for $3 \mathrm{~h}$ at $37^{\circ} \mathrm{C}$. Then, cells were stained with Hoechst 33342 for 15 min, washed with phenol red-free DMEM and imaged on a Nikon Ti2-LAPP microscope with a $60 \times$ objective lens. Fluorescence images were obtained at $405 \mathrm{~nm}$ for Hoechst 33342 and $488 \mathrm{~nm}$ for EGFP. 


\subsection{Intact mass analysis of EGFP-alkyne (26)}

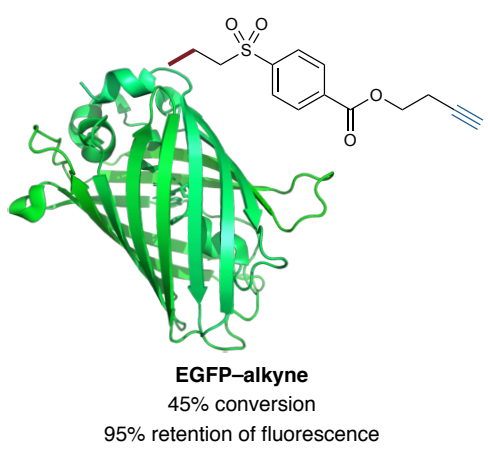

Conversion (45\%) was determined based on intact protein mass analysis.
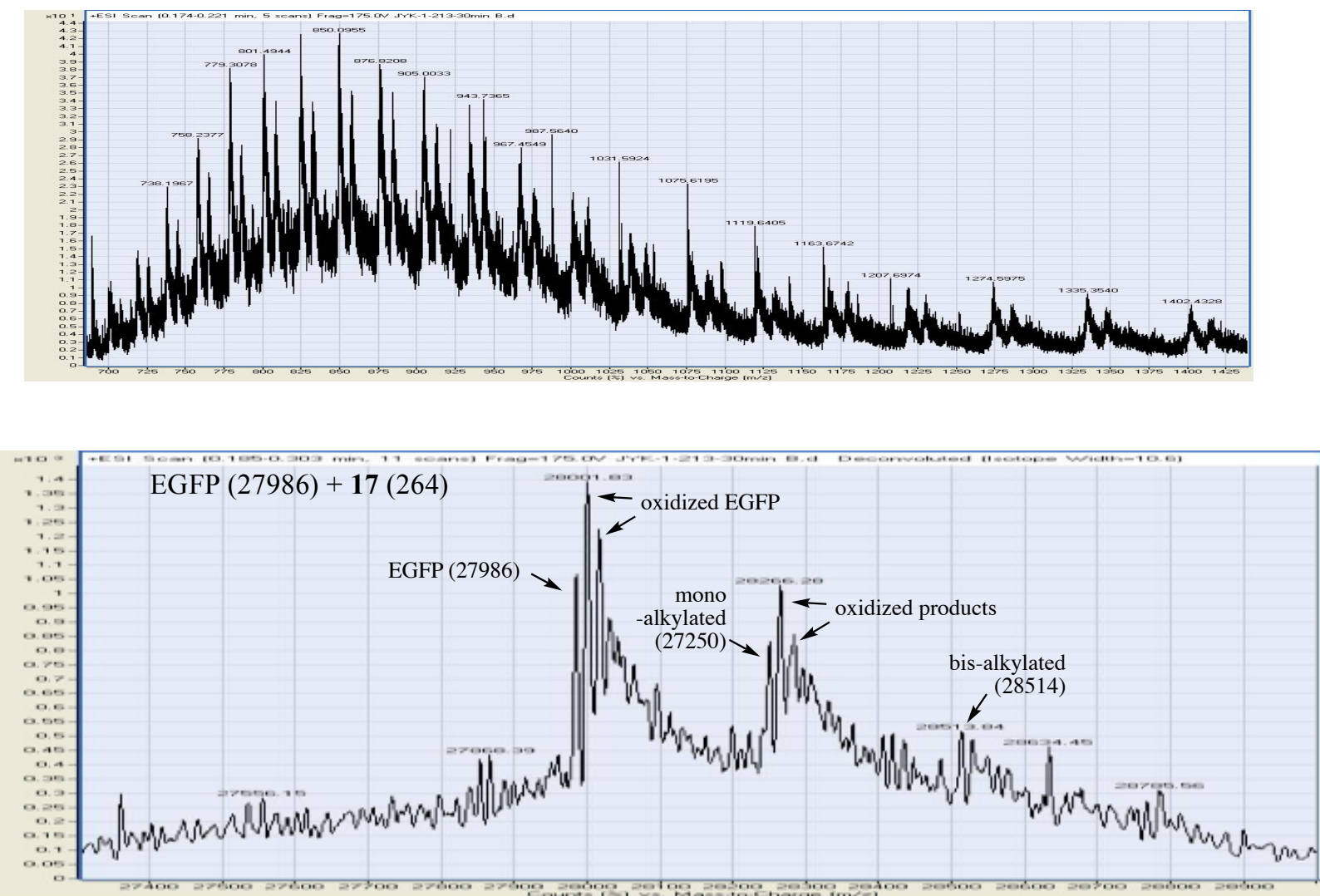


\subsection{Western blot analysis of modified EGFP}
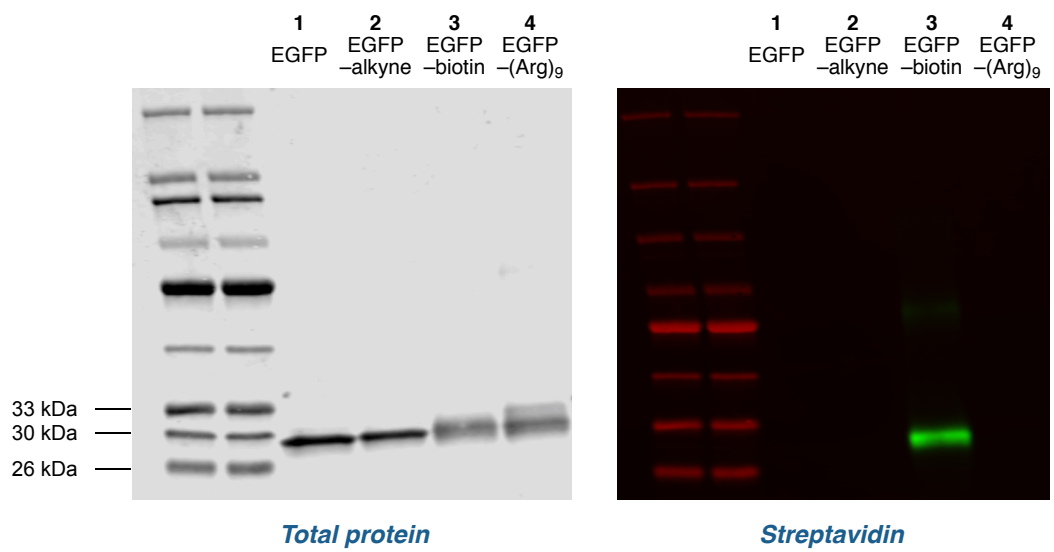

5.4. Confocal microscopy images of protein internalization to SJSA-1 cells

Note: Scale-bars indicate $20 \mu \mathrm{m}$.

EGFP
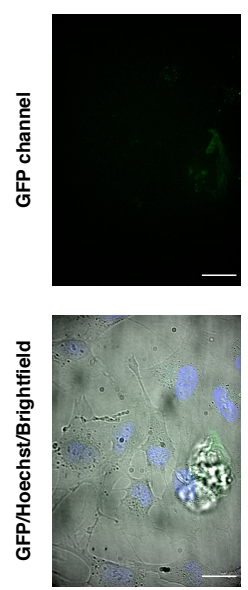
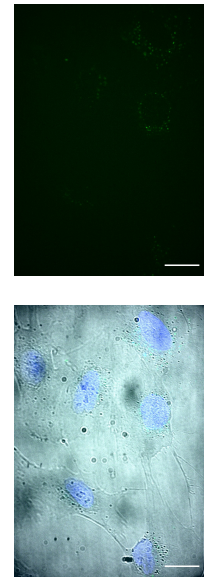
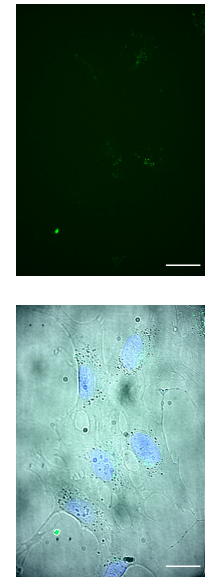

EGFP-alkyne
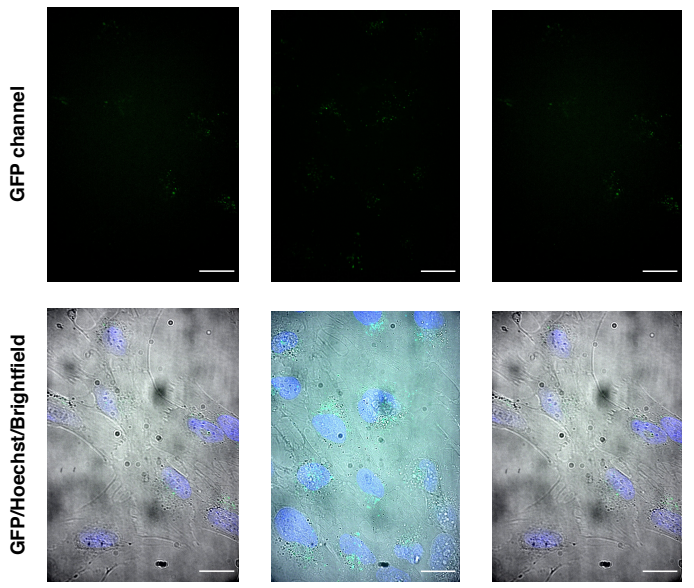

EGFP-(Arg) 9
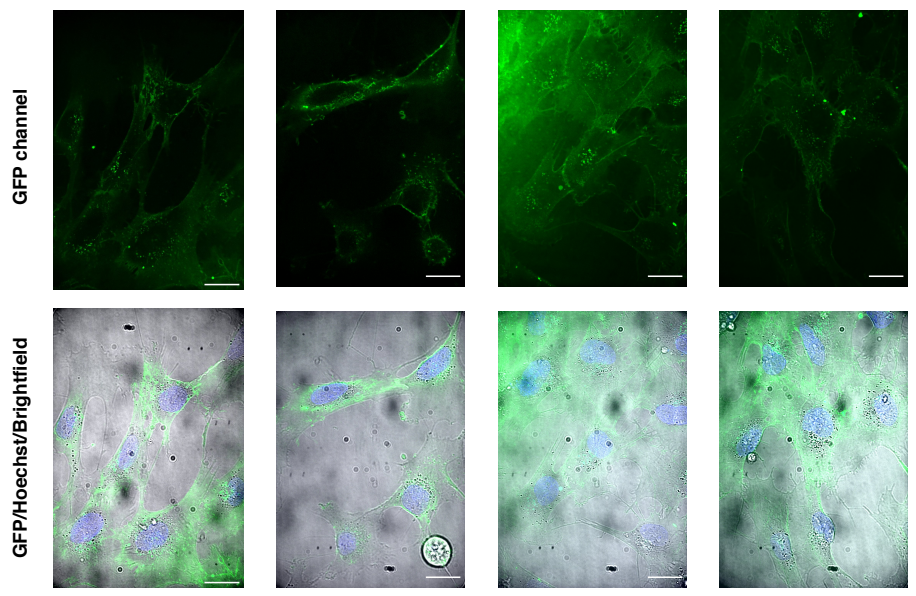


\section{Experimental procedures for the preparation of Michael acceptors}

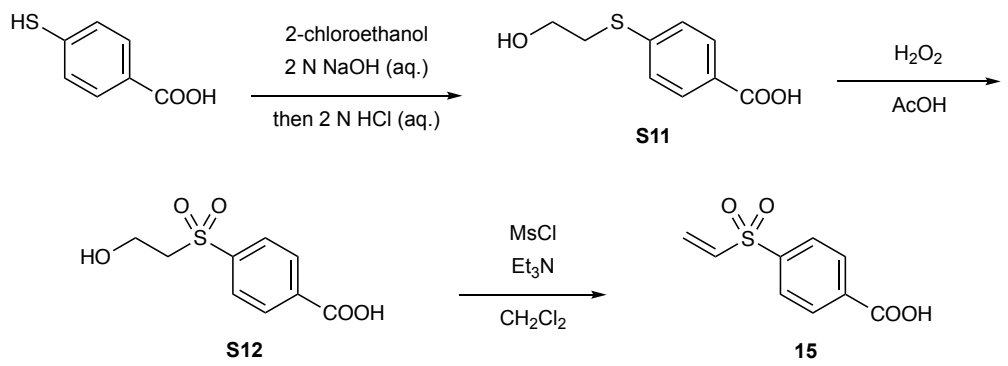

\section{4-(vinylsulfonyl)benzoic acid (15)}

A round bottom flask was charged with 4-mercaptobenzoic acid (3.08 g, $20 \mathrm{mmol}, 1.0$ equiv) in $2 \mathrm{~N}$ aq. $\mathrm{NaOH}$ (20 $\mathrm{mL}$ ). 2-chloroethanol ( $1.5 \mathrm{~mL}, 22 \mathrm{mmol}, 1.1$ equiv) was then added dropwise over $3 \mathrm{~min}$. The reaction was stirred at $40{ }^{\circ} \mathrm{C}$ for $1 \mathrm{~h}$, then cooled to room temperature and stirred for additional $5 \mathrm{~h}$. The reaction was acidified by slow addition of $2 \mathrm{~N}$ aq. $\mathrm{HCl}(50 \mathrm{~mL})$. The aqueous phase was extracted with EtOAc $(3 \times 100 \mathrm{~mL})$ and combined organic extracts were washed with brine, dried over $\mathrm{MgSO}_{4}$ and concentrated under reduced pressure to afford alcohol $\mathbf{S 1 1}$. The crude reaction mixture was carried to the next step without further purification.

A round bottom flask containing alcohol $\mathbf{S 1 1}$ was added acetic acid $(100 \mathrm{~mL})$ and $30 \% \mathrm{H}_{2} \mathrm{O}_{2}$ solution $(20.4 \mathrm{~mL}, 200$ mmol, 10 equiv). Reaction was stirred at room temperature for $16 \mathrm{~h}$ in the absence of light. Solvent was removed under reduced pressure and resulting solid was dissolved in EtOAc $(50 \mathrm{~mL})$ and water $(100 \mathrm{~mL})$. The aqueous phase was extracted with EtOAc $(2 \times 50 \mathrm{~mL})$ and combined organic extracts were washed with brine, dried over $\mathrm{MgSO}_{4}$ and concentrated under reduced pressure to afford sulfone $\mathbf{S 1 2}$. The crude reaction mixture was carried to the next step without further purification.

A round bottom flask containing sulfone $\mathbf{S 1 2}$ was added $\mathrm{CH}_{2} \mathrm{Cl}_{2}(300 \mathrm{~mL})$ and triethylamine $(8.4 \mathrm{~mL}, 60 \mathrm{mmol}, 3$ equiv). Mixture was cooled to $0{ }^{\circ} \mathrm{C}$ and was added methanesulfonyl chloride $(2.3 \mathrm{~mL}, 30 \mathrm{mmol}, 1.5$ equiv) dropwise over $5 \mathrm{~min}$. Then, reaction was stirred for $16 \mathrm{~h}$ at room temperature. Solvent was removed under reduced pressure and resulting solid was purified by flash column chromatography (50-100\% EtOAc in hexanes) to afford desired product 15 (1.11 g, $5.2 \mathrm{mmol}, 26 \%$ over three steps) as an off-white solid.

IR (film): $v_{\max } 3052,2551,1698,1428,1319,1288,1148,1124,748 \mathrm{~cm}^{-1}$.

${ }^{1}$ H NMR (500 MHz, CD 30 ) : $\delta 8.20(\mathrm{~d}, J=8.1 \mathrm{~Hz}, 2 \mathrm{H}), 7.96(\mathrm{~d}, J=8.1 \mathrm{~Hz}, 2 \mathrm{H}), 6.93(\mathrm{dd}, J=16.5,9.9 \mathrm{~Hz}, 1 \mathrm{H})$, $6.46(\mathrm{~d}, J=16.5 \mathrm{~Hz}, 1 \mathrm{H}), 6.17(\mathrm{~d}, J=9.9 \mathrm{~Hz}, 1 \mathrm{H}) \mathrm{ppm}$.

${ }^{13}$ C NMR (125 MHz, $\left.\mathrm{CD}_{3} \mathrm{OD}\right): \delta 170.0,144.1,139.7,131.5,129.6,129.2,128.8 \mathrm{ppm}$.

HRMS (ESI) $m / z$ calcd. for $\mathrm{C}_{9} \mathrm{H}_{9} \mathrm{O}_{4} \mathrm{~S}^{+}\left[\mathrm{M}+\mathrm{H}^{+}\right] 213.0216$, found 213.0217 . 


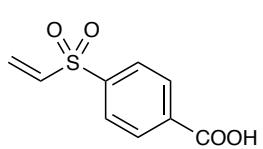

15

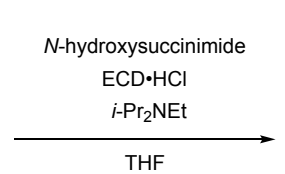

THF

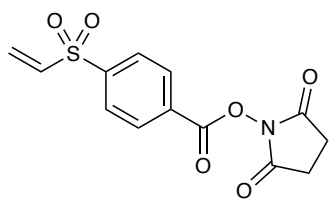

S13

\section{2,5-dioxopyrrolidin-1-yl 4-(vinylsulfonyl)benzoate (S13)}

An $8 \mathrm{~mL}$ vial charged with 15 (212 mg, $1 \mathrm{mmol}, 1.0$ equiv), $N$-hydroxysuccinimide (115 mg, $1 \mathrm{mmol}, 1$ equiv), and (3-dimethylaminopropyl)- $N^{\prime}$-ethylcarbodiimide hydrochloride (192 mg, $1 \mathrm{mmol}, 1$ equiv) was added THF (4 mL) and $i-\operatorname{Pr}_{2} \mathrm{NEt}(0.18 \mathrm{~mL}, 1 \mathrm{mmol}, 1$ equiv). The reaction was stirred at room temperature for $3 \mathrm{~h}$. Then, the reaction was diluted with EtOAc $(10 \mathrm{~mL})$ and water $(10 \mathrm{~mL})$. The aqueous phase was extracted with EtOAc $(2 \times 10 \mathrm{~mL})$ and combined organic extracts were washed with brine, dried over $\mathrm{MgSO}_{4}$ and concentrated under reduced pressure and crude reaction mixture was purified by flash column chromatography (30-80\% EtOAc in hexanes) to afford desired product $\mathbf{S 1 3}$ (243 $\mathrm{mg}, 0.79 \mathrm{mmol}, 79 \%$ yield) as a white solid.

IR (film): $v_{\max } 2958,1774,1737,1321,1202,1150,1067,1002,774 \mathrm{~cm}^{-1}$.

${ }^{1} \mathbf{H}$ NMR $\left(500 \mathrm{MHz}, \mathrm{CDCl}_{3}\right): \delta 8.33(\mathrm{~d}, J=8.6 \mathrm{~Hz}, 2 \mathrm{H}), 8.07(\mathrm{~d}, J=8.6 \mathrm{~Hz}, 2 \mathrm{H}), 6.69(\mathrm{dd}, J=16.5,9.6 \mathrm{~Hz}, 1 \mathrm{H})$, $6.58(\mathrm{~d}, J=16.5 \mathrm{~Hz}, 1 \mathrm{H}), 6.18(\mathrm{~d}, J=9.6 \mathrm{~Hz}, 1 \mathrm{H}), 2.95(\mathrm{~s}, 4 \mathrm{H}) \mathrm{ppm}$.

${ }^{13}$ C NMR (125 MHz, $\left.\mathrm{CDCl}_{3}\right): \delta$ 168.9, 160.7, 145.5, 137.7, 131.6, 129.96, 129.93, 128.5, $25.8 \mathrm{ppm}$.

HRMS (ESI) $m / z$ calcd. for $\mathrm{C}_{13} \mathrm{H}_{12} \mathrm{NO}_{6} \mathrm{SNa}^{+}\left[\mathrm{M}+\mathrm{Na}^{+}\right] 332.0199$, found 332.0202 .

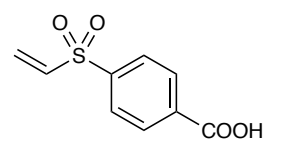

15

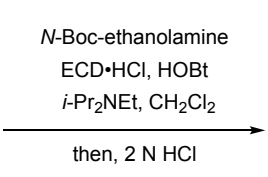

then, $2 \mathrm{~N} \mathrm{HCl}$

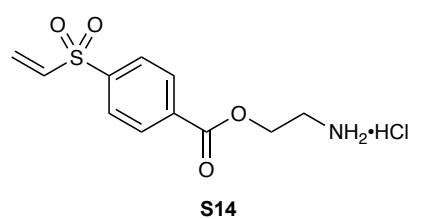

\section{2-aminoethyl 4-(vinylsulfonyl)benzoate hydrochloride (S14)}

An $8 \mathrm{~mL}$ vial charged with 15 (200 mg, $0.94 \mathrm{mmol}, 1.0$ equiv), $N$-Boc-aminoethanol (167 mg, $1.04 \mathrm{mmol}, 1.1$ equiv), (3-dimethylaminopropyl)- $N^{\prime}$-ethylcarbodiimide hydrochloride (217 mg, $1.13 \mathrm{mmol}, 1.2$ equiv), and 1Hydroxybenzotriazole hydrate $\left(173 \mathrm{mg}, 1.13 \mathrm{mmol}, 1.2\right.$ equiv) was added $\mathrm{CH}_{2} \mathrm{Cl}_{2}(4 \mathrm{~mL})$ and $i-\mathrm{Pr}_{2} \mathrm{NEt}(0.33 \mathrm{~mL}$, $1.88 \mathrm{mmol}, 2$ equiv). The reaction was stirred at room temperature for $15 \mathrm{~h}$. Then, the reaction was diluted with $\mathrm{CH}_{2} \mathrm{Cl}_{2}(5 \mathrm{~mL})$ and water $(10 \mathrm{~mL})$. The aqueous phase was extracted with $\mathrm{CH}_{2} \mathrm{Cl}_{2}(2 \times 10 \mathrm{~mL})$ and combined organic extracts were dried over $\mathrm{MgSO}_{4}$ and concentrated under reduced pressure. Resulting crude reaction mixture was dissolved in $2 \mathrm{~N} \mathrm{HCl}(1 \mathrm{~mL})$ and directly purified by reverse phase flash column chromatography $(0-20 \% \mathrm{MeCN}$ in water) to afford desired product $\mathbf{S 1 4}$ (90 $\mathrm{mg}, 0.31 \mathrm{mmol}, 33 \%$ yield) as a white solid.

IR (film): $v_{\max } 3399,2960,1724,1401,1275,1148,1111,979,751 \mathrm{~cm}^{-1}$.

${ }^{1}$ H NMR (500 MHz, CD $\left.{ }_{3} \mathrm{OD}\right): \delta 8.33(\mathrm{~d}, J=8.6 \mathrm{~Hz}, 2 \mathrm{H}), 8.04(\mathrm{~d}, J=8.6 \mathrm{~Hz}, 2 \mathrm{H}), 6.96(\mathrm{dd}, J=16.4,9.9 \mathrm{~Hz}, 1 \mathrm{H})$, $6.49(\mathrm{~d}, J=16.4 \mathrm{~Hz}, 1 \mathrm{H}), 6.22(\mathrm{~d}, J=9.9 \mathrm{~Hz}, 1 \mathrm{H}), 4.64-4.58(\mathrm{~m}, 2 \mathrm{H}), 3.41$ (t, $J=5.1 \mathrm{~Hz}, 2 \mathrm{H}) \mathrm{ppm}$.

${ }^{13}$ C NMR (125 MHz, $\left.\mathrm{CD}_{3} \mathrm{OD}\right): \delta 166.1,145.6,139.3,135.4,131.9,130.2,129.1,63.1,39.9 \mathrm{ppm}$.

HRMS (ESI) $m / z$ calcd. for $\mathrm{C}_{11} \mathrm{H}_{14} \mathrm{NO}_{4} \mathrm{~S}^{+}\left[\mathrm{M}-\mathrm{Cl}^{-}\right] 256.0638$, found 256.0632 . 


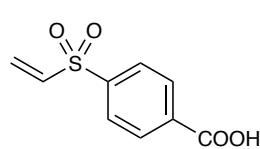

15

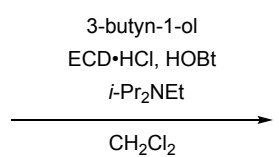

$\mathrm{CH}_{2} \mathrm{Cl}_{2}$

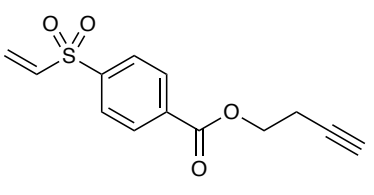

17

\section{But-3-yn-1-yl 4-(vinylsulfonyl)benzoate (17)}

An $8 \mathrm{~mL}$ vial charged with 15 (200 mg, $0.94 \mathrm{mmol}, 1.0$ equiv), 3-butyn-1-ol (73 mg, $1.04 \mathrm{mmol}, 1.1$ equiv), (3dimethylaminopropyl)- $N$ '-ethylcarbodiimide hydrochloride $(217 \mathrm{mg}, 1.13 \mathrm{mmol}, 1.2$ equiv), and 1Hydroxybenzotriazole hydrate (173 mg, $1.13 \mathrm{mmol}, 1.2$ equiv) was added $\mathrm{CH}_{2} \mathrm{Cl}_{2}(4 \mathrm{~mL})$ and $i-\mathrm{Pr}_{2} \mathrm{NEt}(0.33 \mathrm{~mL}$, $1.88 \mathrm{mmol}, 2$ equiv). The reaction was stirred at room temperature for $15 \mathrm{~h}$. Then, the reaction was diluted with $\mathrm{CH}_{2} \mathrm{Cl}_{2}(5 \mathrm{~mL})$ and water $(10 \mathrm{~mL})$. The aqueous phase was extracted with $\mathrm{CH}_{2} \mathrm{Cl}_{2}(2 \times 10 \mathrm{~mL})$ and combined organic extracts were dried over $\mathrm{MgSO}_{4}$ and concentrated under reduced pressure. Resulting crude reaction mixture was purified by flash column chromatography (30-100\% EtOAc in hexanes) to afford desired product 17 (111 mg, 0.42 $\mathrm{mmol}, 45 \%$ yield) as a clear oil.

IR (film): $v_{\max } 3281,1723,1399,1272,1150,1118,1015,750 \mathrm{~cm}^{-1}$.

${ }^{1}$ H NMR $\left(500 \mathrm{MHz}, \mathrm{CDCl}_{3}\right): \delta 8.24(\mathrm{~d}, J=8.4 \mathrm{~Hz}, 2 \mathrm{H}), 8.00(\mathrm{~d}, J=8.4 \mathrm{~Hz}, 2 \mathrm{H}), 6.68(\mathrm{dd}, J=16.5,9.7 \mathrm{~Hz}, 1 \mathrm{H})$, $6.55(\mathrm{~d}, J=16.5 \mathrm{~Hz}, 1 \mathrm{H}), 6.14(\mathrm{~d}, J=9.7 \mathrm{~Hz}, 1 \mathrm{H}), 4.48(\mathrm{t}, J=6.7 \mathrm{~Hz}, 2 \mathrm{H}), 2.71(\mathrm{td}, J=6.7,2.7 \mathrm{~Hz}, 2 \mathrm{H}), 2.06(\mathrm{t}, J$ $=2.6 \mathrm{~Hz}, 1 \mathrm{H}) \mathrm{ppm}$.

${ }^{13}$ C NMR $\left(125 \mathrm{MHz}, \mathrm{CDCl}_{3}\right): \delta 164.8,143.7,138.0,134.7,130.7,129.2,128.1,79.8,70.4,63.4,19.2 \mathrm{ppm}$.

HRMS (ESI) $m / z$ calcd. for $\mathrm{C}_{13} \mathrm{H}_{13} \mathrm{O}_{4} \mathrm{~S}^{+}\left[\mathrm{M}+\mathrm{H}^{+}\right] 265.0529$, found 265.0528 .
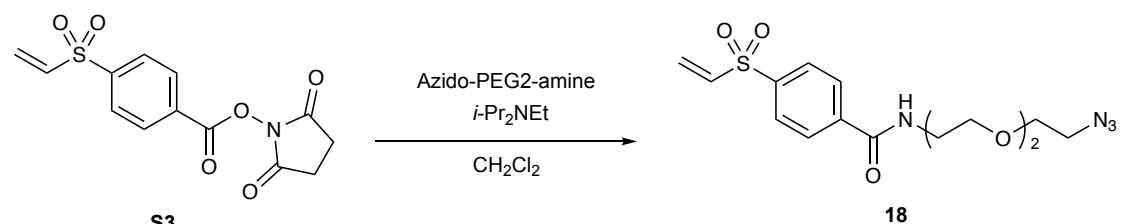

\section{$N$-(2-(2-(2-azidoethoxy)ethoxy)ethyl)-4-(vinylsulfonyl)benzamide (18)}

An $8 \mathrm{~mL}$ vial charged with $\mathbf{S 3}$ (7.1 mg, $23 \mu \mathrm{mol}, 1.0$ equiv), azido-PEG2-amine (4.0 mg, $23 \mu \mathrm{mol}, 1.0$ equiv) was added $\mathrm{CH}_{2} \mathrm{Cl}_{2}(1 \mathrm{~mL})$ and $i$ - $\operatorname{Pr}_{2} \mathrm{NEt}(2.0 \mu \mathrm{L}, 12 \mu \mathrm{mol}, 0.5$ equiv). The reaction was stirred at room temperature for 2 $\mathrm{h}$. Then, the reaction was concentrated under reduced pressure. Resulting crude reaction mixture was purified by flash column chromatography $\left(0-10 \% \mathrm{MeOH}\right.$ in $\left.\mathrm{CH}_{2} \mathrm{Cl}_{2}\right)$ to afford desired product $\mathbf{1 8}$ (4.2 $\mathrm{mg}, 11 \mu \mathrm{mol}, 50 \%$ yield) as a clear oil.

IR (film): $v_{\max } 3349,2874,2103,1653,1541,1301,1086,749 \mathrm{~cm}^{-1}$.

${ }^{1} \mathbf{H}$ NMR $\left(500 \mathrm{MHz}, \mathrm{CDCl}_{3}\right): \delta 7.96(\mathrm{~s}, 4 \mathrm{H}), 6.80(\mathrm{~s}, 1 \mathrm{H}), 6.66(\mathrm{dd}, J=16.4,9.6 \mathrm{~Hz}, 1 \mathrm{H}), 6.51(\mathrm{~d}, J=16.4 \mathrm{~Hz}, 1 \mathrm{H})$, $6.10(\mathrm{~d}, J=9.6 \mathrm{~Hz}, 1 \mathrm{H}), 3.71-3.65(\mathrm{~m}, 10 \mathrm{H}), 3.37(\mathrm{t}, J=4.9 \mathrm{~Hz}, 2 \mathrm{H}) \mathrm{ppm}$. 
${ }^{13}$ C NMR (125 MHz, $\left.\mathrm{CDCl}_{3}\right): \delta 166.0,142.3,139.7,138.3,128.9,128.4,128.3,70.7,70.5,70.3,69.9,50.8,40.2$ ppm.

HRMS (ESI) $m / z$ calcd. for $\mathrm{C}_{15} \mathrm{H}_{20} \mathrm{~N}_{4} \mathrm{O}_{5} \mathrm{NaS}^{+}\left[\mathrm{M}+\mathrm{Na}^{+}\right]$391.1047, found 391.1045.

Note: Neat 18 undergoes slow alkene-azide 1,3-dipolar cycloaddition.

For long-term storage, 18 was stored as a solution in benzene at $-20{ }^{\circ} \mathrm{C}$.

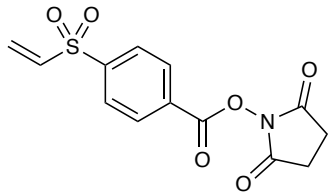

S3

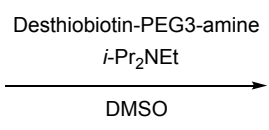

DMSO

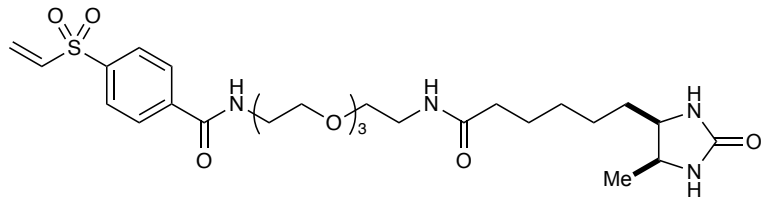

19

\section{$N$-(18-((4R,5S)-5-methyl-2-oxoimidazolidin-4-yl)-13-oxo-3,6,9-trioxa-12-azaoctadecyl)-4-}

\section{(vinylsulfonyl)benzamide (19)}

An $8 \mathrm{~mL}$ vial charged with $\mathbf{S 3}$ ( $16 \mathrm{mg}, 52 \mu \mathrm{mol}, 1.0$ equiv), desthiobiotin-PEG3-amine ( $20 \mathrm{mg}, 51 \mu \mathrm{mol}, 1.0$ equiv) was added DMSO $(1 \mathrm{~mL})$ and $i-\operatorname{Pr}_{2} \mathrm{NEt}(4.5 \mu \mathrm{L}, 26 \mu \mathrm{mol}, 0.5$ equiv). The reaction was stirred at room temperature for $16 \mathrm{~h}$. Then, the reaction was concentrated under reduced pressure using genevac. Resulting crude reaction mixture was purified by flash column chromatography $\left(0-10 \% \mathrm{MeOH}\right.$ in $\left.\mathrm{CH}_{2} \mathrm{Cl}_{2}\right)$ to afford desired product 19 (20 $\mathrm{mg}, 34 \mu \mathrm{mol}, 65 \%$ yield) as a clear oil.

IR (film): $v_{\max } 3302,2932,1694,1648,1547,1317,1144,1088,750 \mathrm{~cm}^{-1}$.

${ }^{1}$ H NMR (500 MHz, $\left.\mathrm{CDCl}_{3}\right): \delta 8.03(\mathrm{~d}, J=8.4 \mathrm{~Hz}, 2 \mathrm{H}), 7.93(\mathrm{~d}, J=8.4 \mathrm{~Hz}, 2 \mathrm{H}), 7.69(\mathrm{t}, J=5.3 \mathrm{~Hz}, 1 \mathrm{H}), 6.67(\mathrm{dd}$, $J=16.5,9.8 \mathrm{~Hz}, 1 \mathrm{H}), 6.55$ (t, $J=5.7 \mathrm{~Hz}, 1 \mathrm{H}), 6.49$ (d, $J=16.5 \mathrm{~Hz}, 1 \mathrm{H}), 6.10(\mathrm{~d}, J=9.8 \mathrm{~Hz}, 1 \mathrm{H}), 5.70(\mathrm{~s}, 1 \mathrm{H}), 4.89$ (s, 1H), 3.81 (quint, $J=6.8 \mathrm{~Hz}, 1 \mathrm{H}), 3.72-3.55(\mathrm{~m}, 13 \mathrm{H}), 3.51(\mathrm{t}, J=5.2 \mathrm{~Hz}, 2 \mathrm{H}), 3.38(\mathrm{q}, J=5.3 \mathrm{~Hz}, 2 \mathrm{H}), 2.16(\mathrm{t}, J$ $=7.4 \mathrm{~Hz}, 2 \mathrm{H}$ ), 1.61 (quint, $J=7.1 \mathrm{~Hz}, 2 \mathrm{H}), 1.51-1.16(\mathrm{~m}, 4 \mathrm{H}), 1.21-1.06(\mathrm{~m}, 4 \mathrm{H}) \mathrm{ppm}$.

${ }^{13}$ C NMR (125 MHz, $\left.\mathrm{CDCl}_{3}\right): \delta 173.4,166.1,164.0,142.0,139.6,138.1,128.9,128.5,128.2,70.5,70.4,70.2,70.1$, $70.0,69.8,56.1,51.5,40.2,39.2,36.0,29.5,28.6,25.9,25.3,15.9 \mathrm{ppm}$.

HRMS (ESI) $m / z$ calcd. for $\mathrm{C}_{27} \mathrm{H}_{42} \mathrm{~N}_{4} \mathrm{O}_{8} \mathrm{NaS}^{+}\left[\mathrm{M}+\mathrm{Na}^{+}\right]$605.2616, found 605.2615. 
7. Spectral data
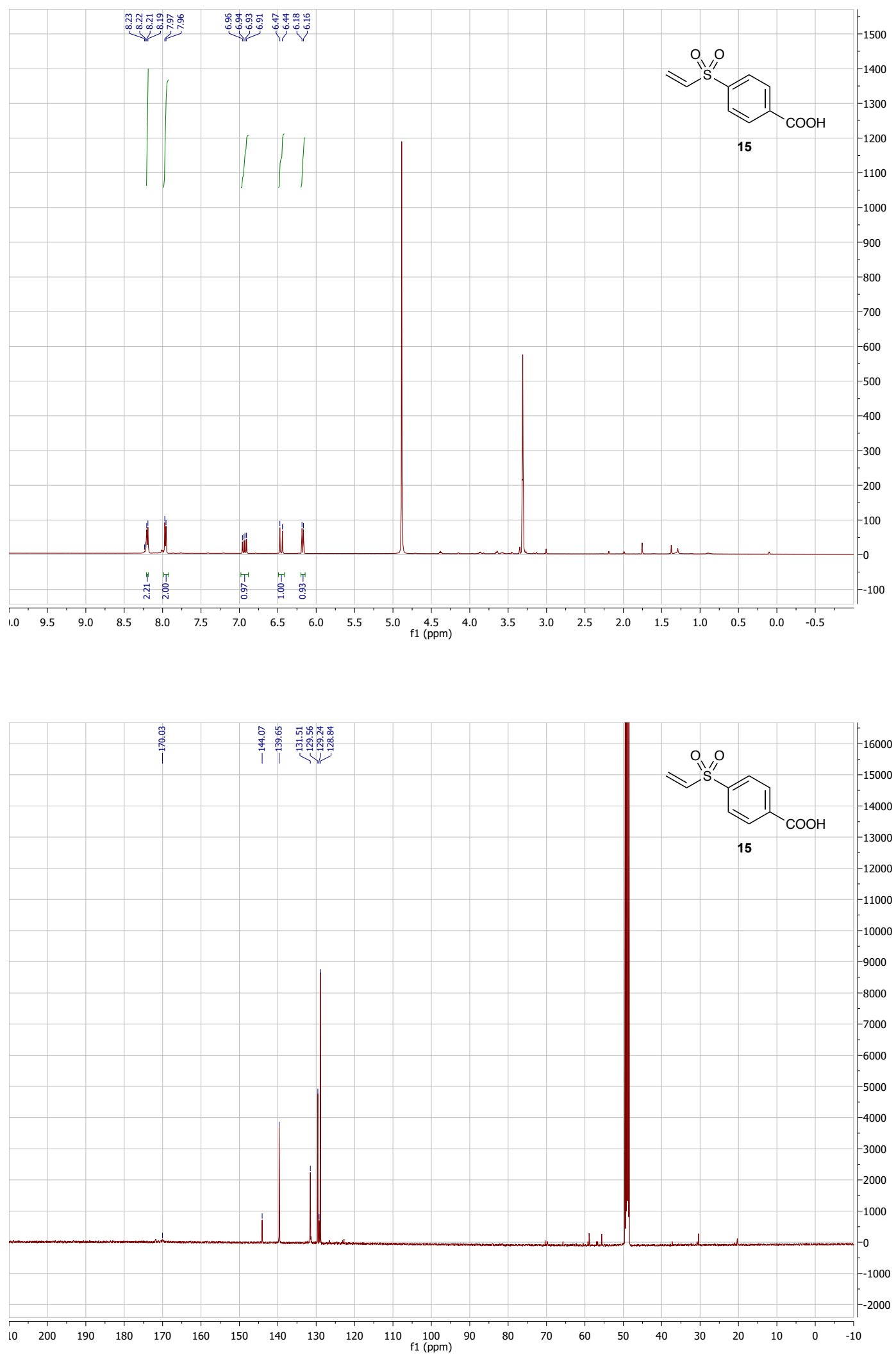

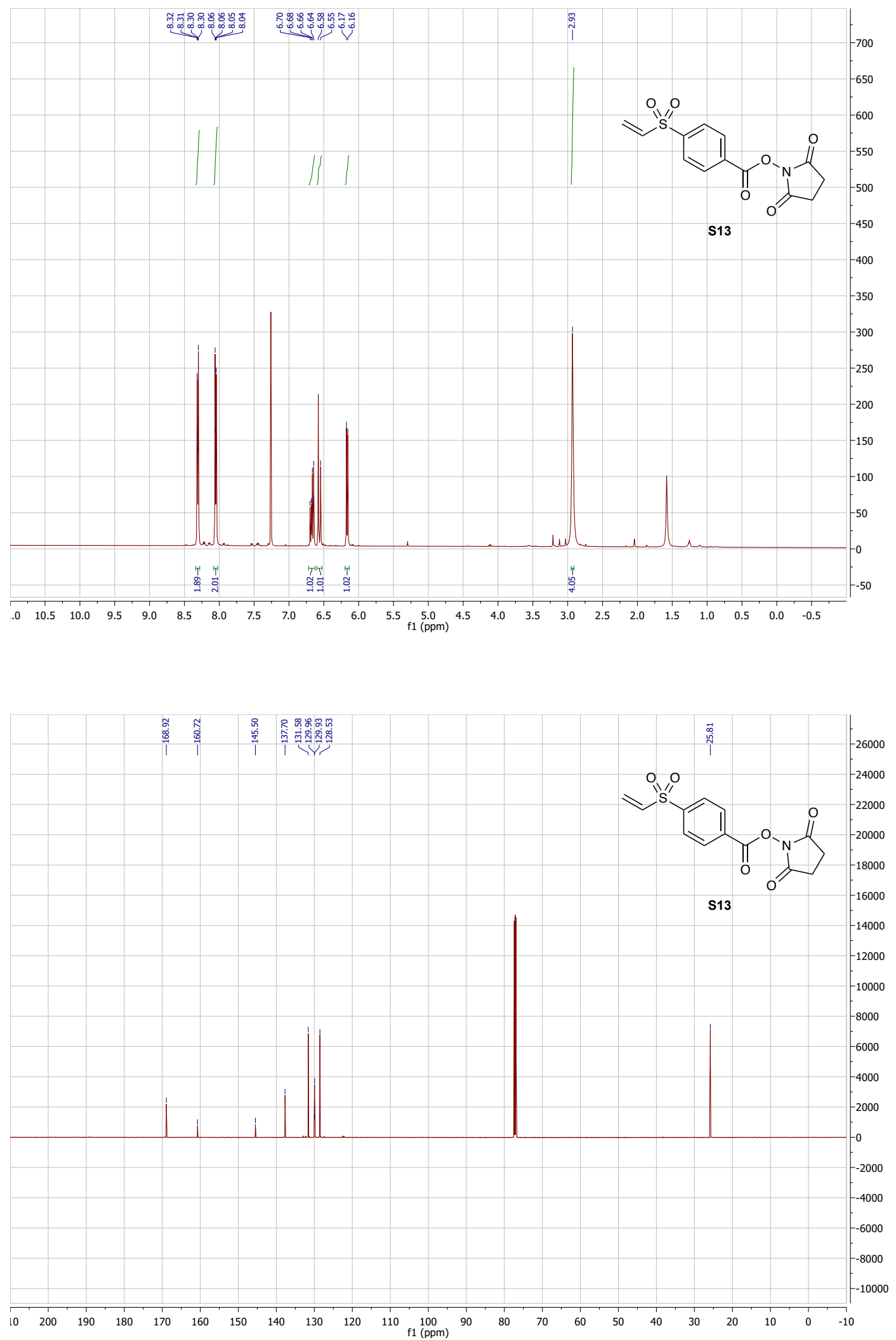

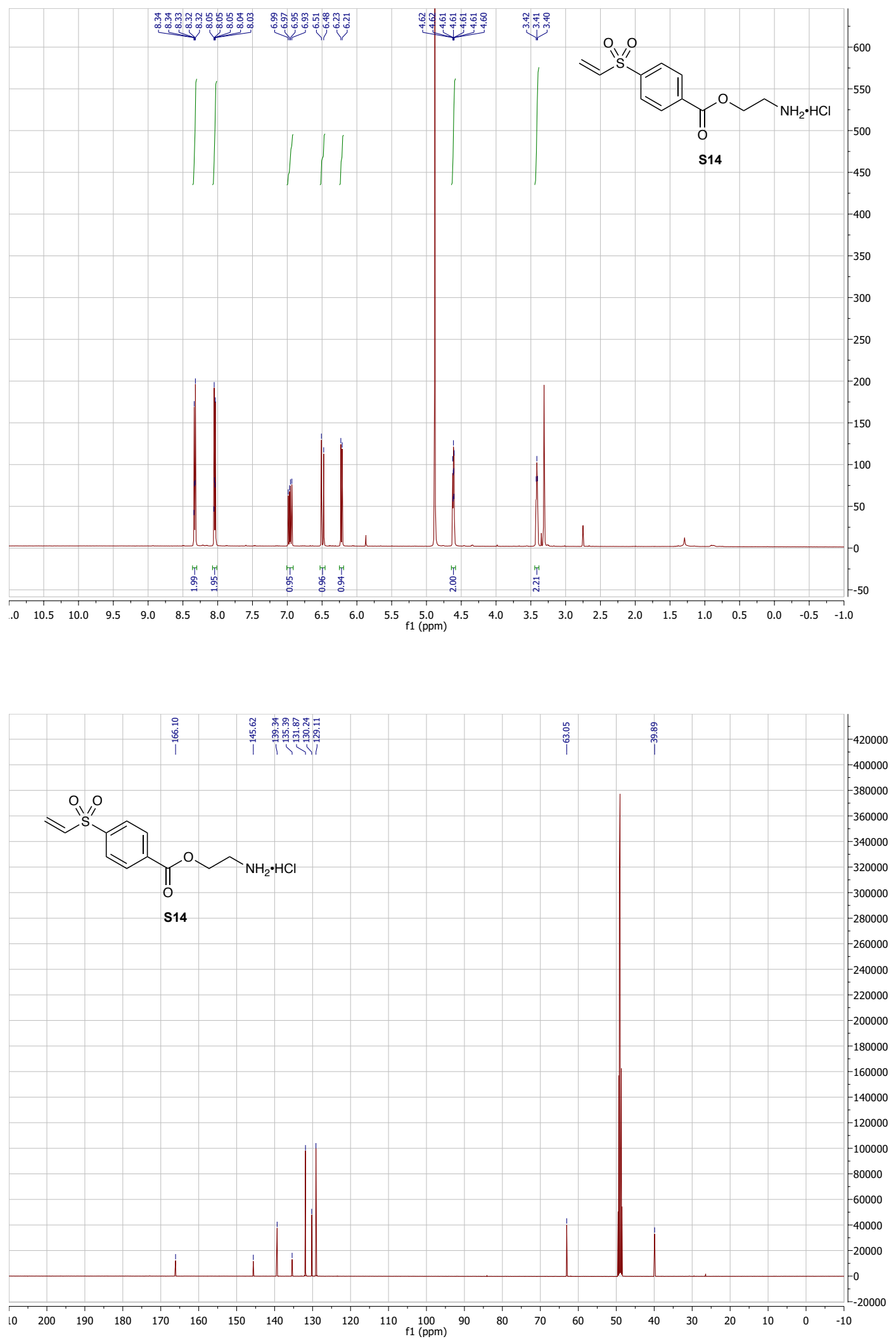

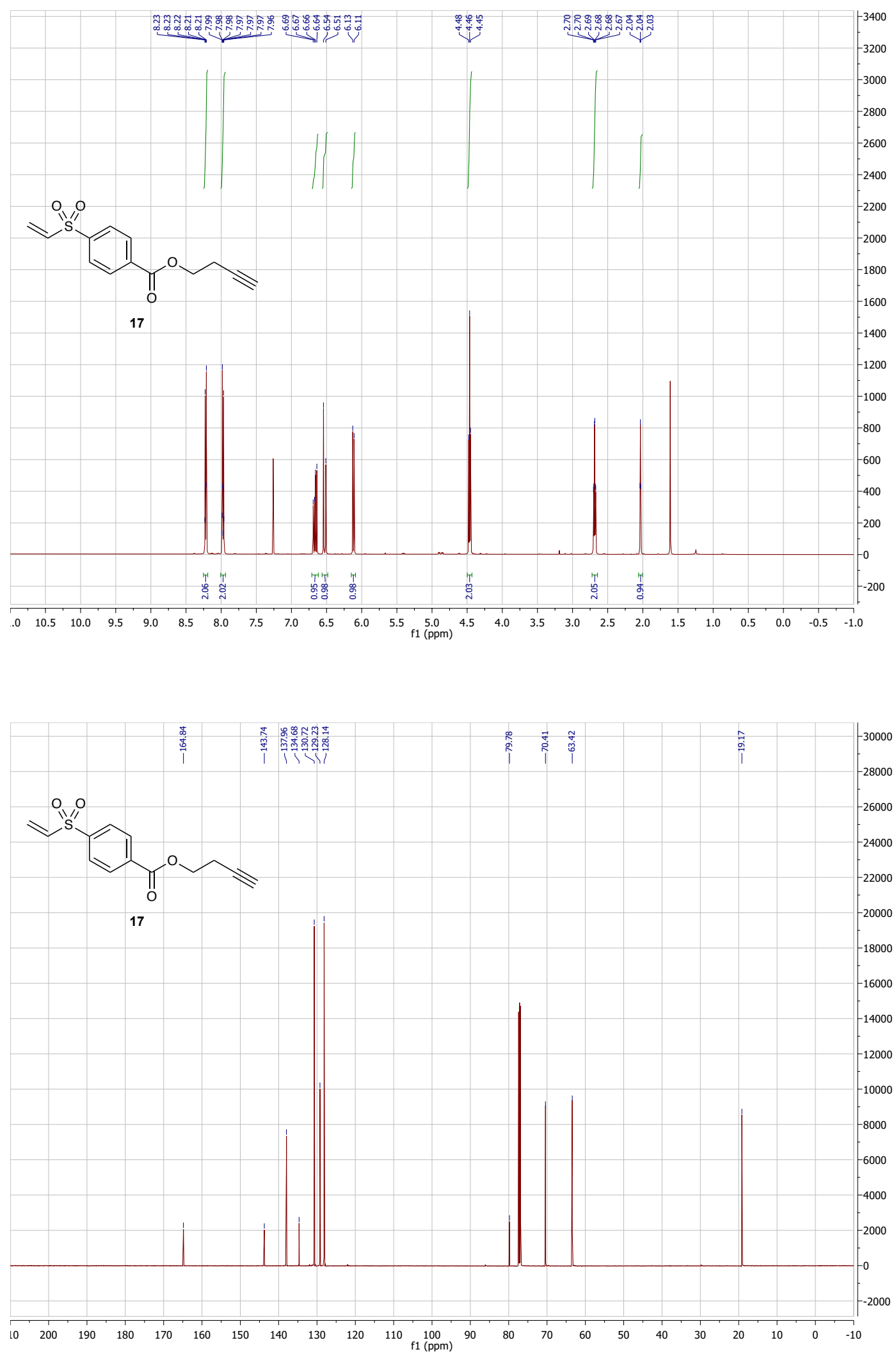

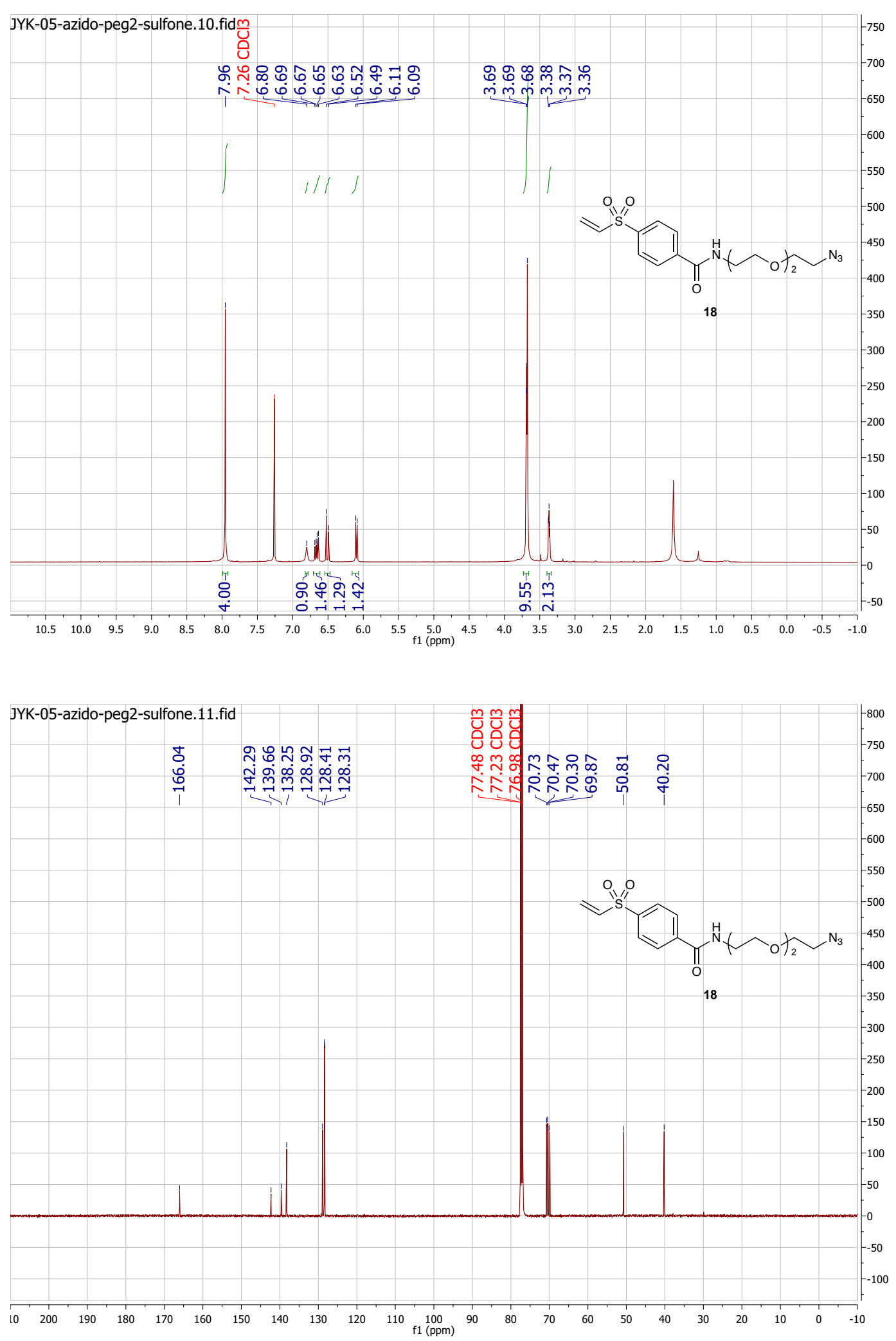

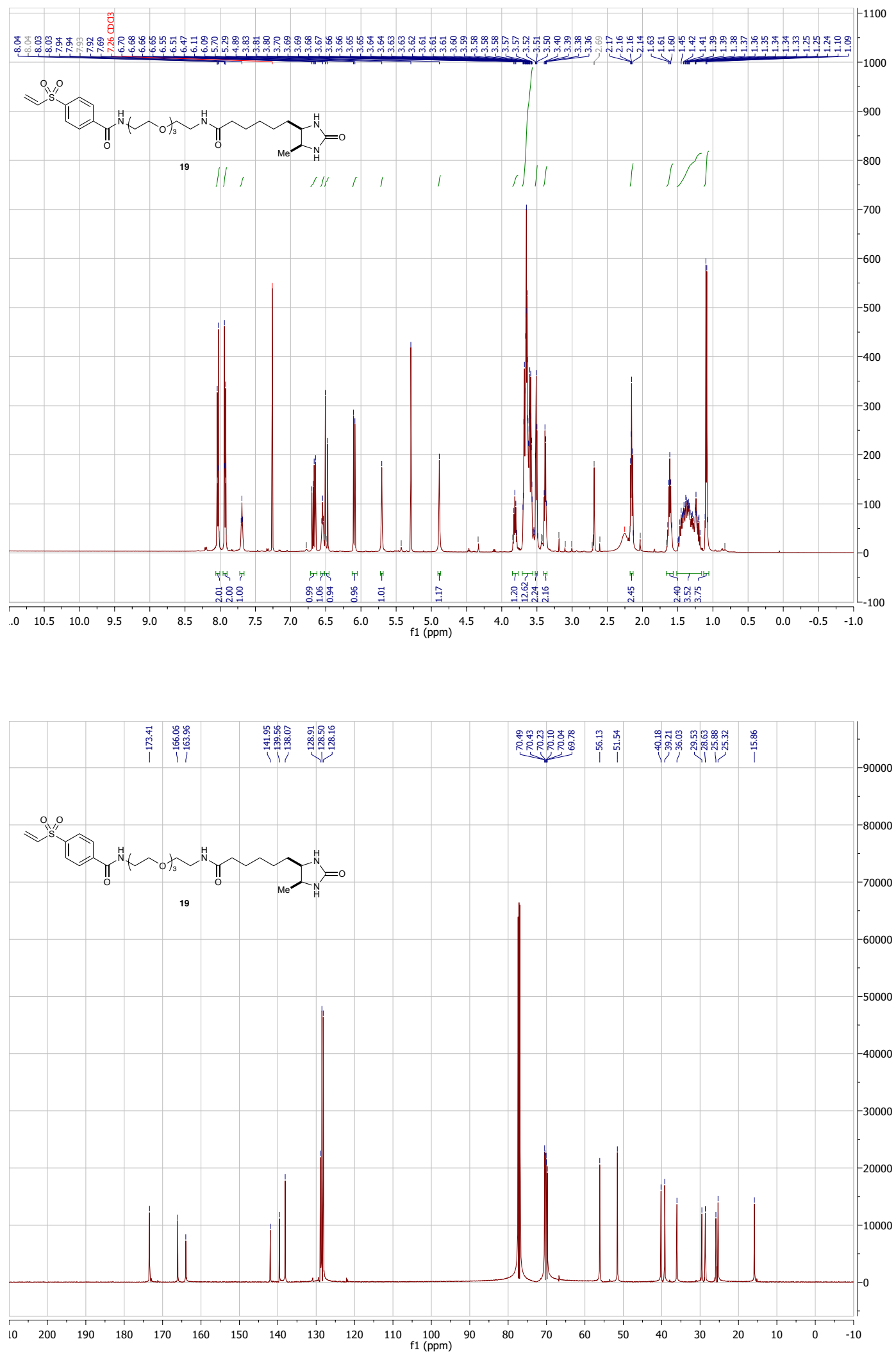


\section{Cyclic voltammetry data of amino acids}

Cyclic voltammetry was performed on a $\mathrm{CH}$ Instruments Electrochemical Analyzer (CHI600E). A $5 \mathrm{mM}$ solution of an amino acid was prepared in PBS (Gibco, 1x, pH 7.4) and sparged with $\mathrm{N}_{2}$ for 15 minutes. The cyclic voltammogram was obtained using a glassy carbon working electrode, a Pt counter electrode, and a saturated calomel reference electrode. Scan rate $=0.05 \mathrm{~V} / \mathrm{s}$.
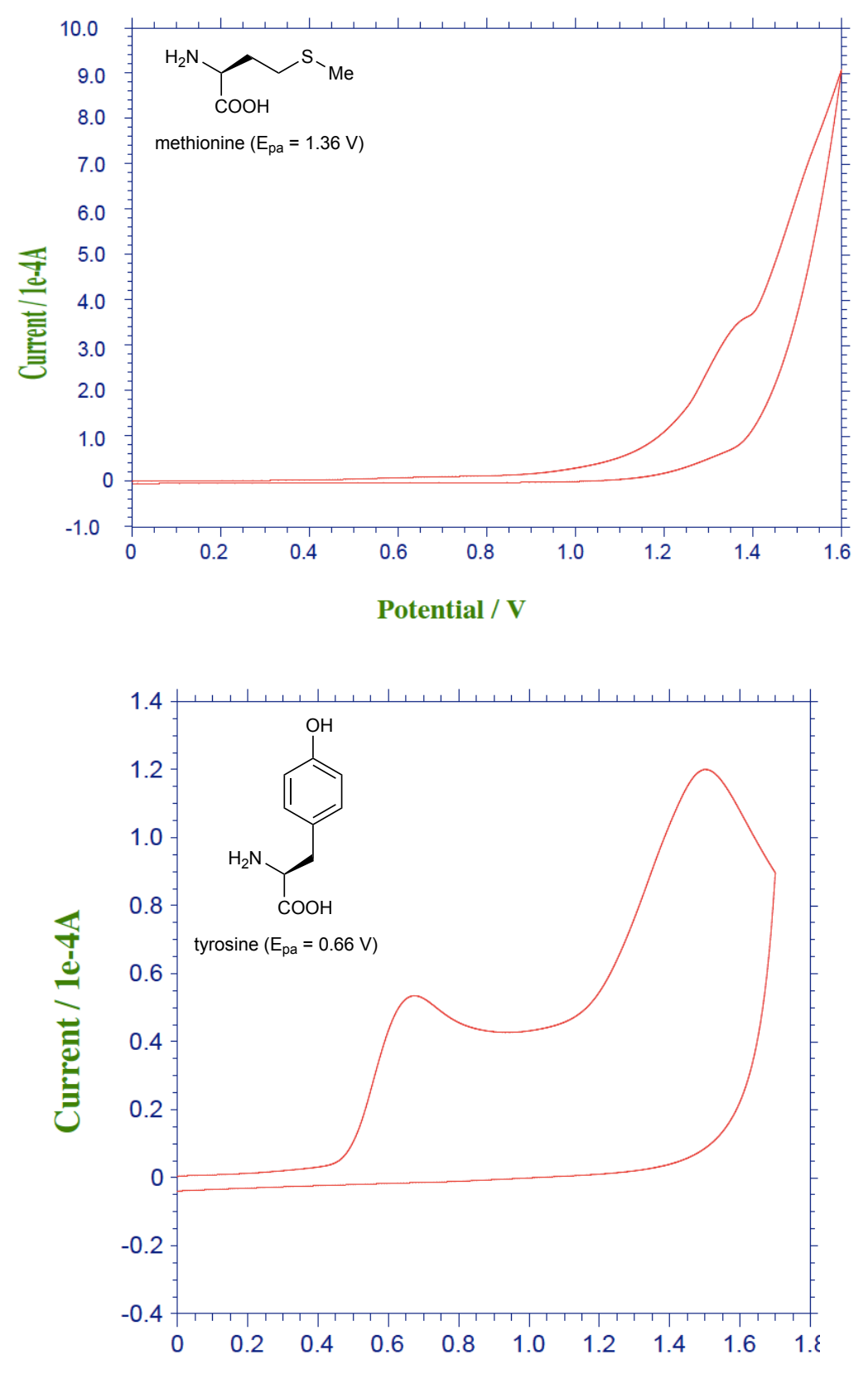

Potential / V 


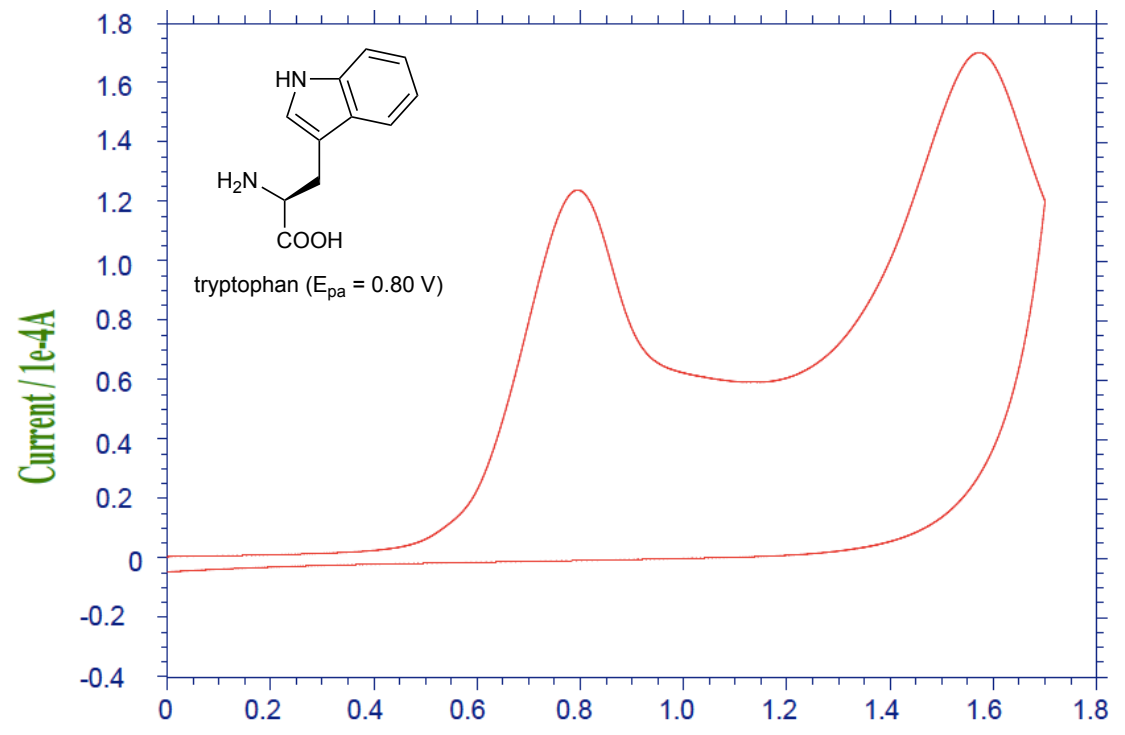

Potential / V

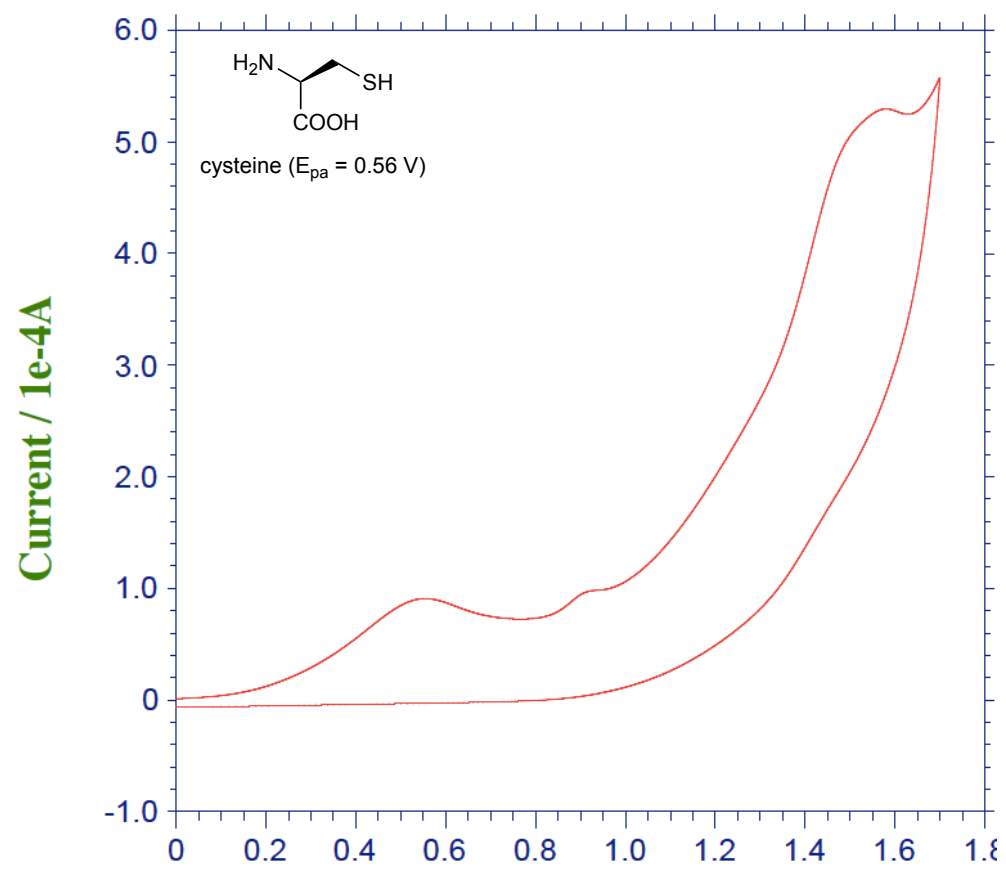

Potential / V 


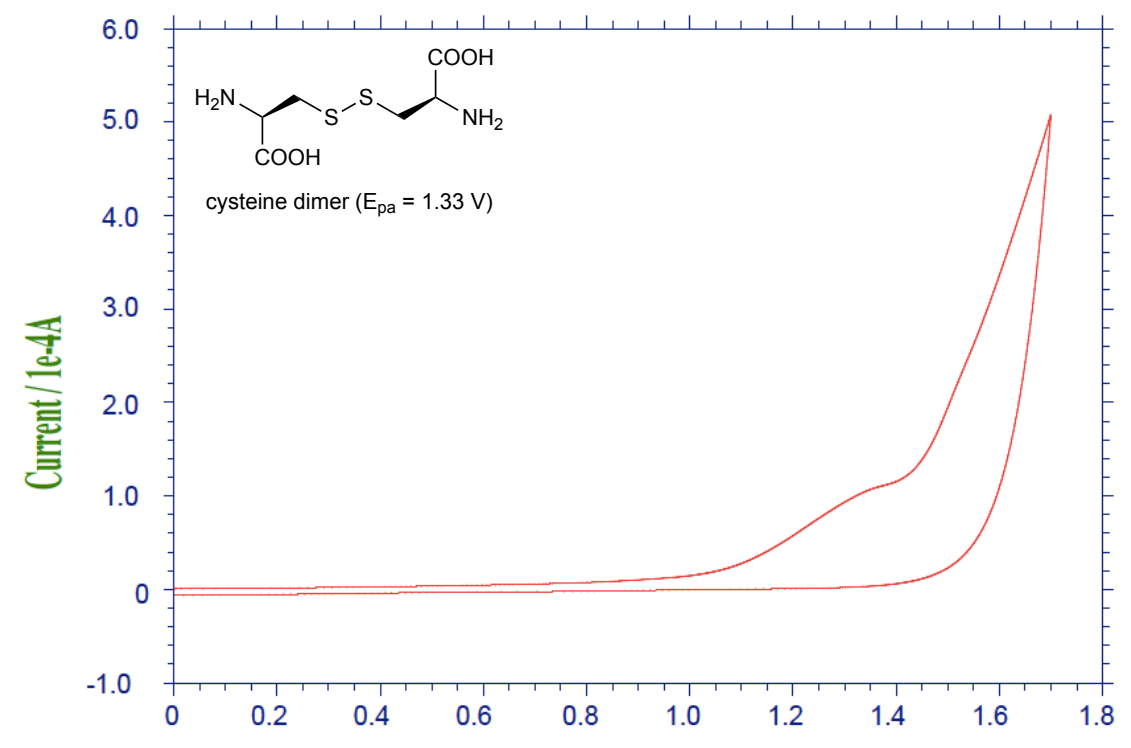

Potential / V 


\section{References}

1. Bloom, S.; Liu, C.; Kölmel, D. K.; Qiao, J. X.; Zhang, Y.; Poss, M. A.; Ewing, W. R.; MacMillan, D. W. C. Nat. Chem. 2018, 10, 205-211.

2. Presolski, S. I.; Hong, V.; Finn, M. G. Curr. Protoc. Chem. Biol. 2011, 3, 153-162.

3. Jia, S.; He, D.; Chang, C. J. J. Am. Chem. Soc. 2019, 141, 7294-7301. 